\title{
Interference from Large Wireless Networks under Correlated Shadowing
}

by

Sebastian S. Szyszkowicz, B.A.Sc., M.A.Sc.

A thesis submitted to The Faculty of Graduate and Postdoctoral Affairs in partial fulfillment of the requirements for the degree of Doctor of Philosophy in Electrical and Computer Engineering

Ottawa-Carleton Institute for Electrical and Computer Engineering (OCIECE) Department of Systems and Computer Engineering Carleton University

Ottawa, Ontario, Canada, K1S 5B6

January 2011

(C) Copyright 2011, Sebastian S. Szyszkowicz 


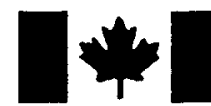

Library and Archives
Canada

Published Heritage

Branch

395 Wellington Street Ottawa ON K1A ON4 Canada
Bibliothèque et

Archives Canada

Direction du

Patrimoine de l'édition

395 , rue Wellington

Ottawa ON K1A ON4

Canada
Your file Votre référence
ISBN: $978-0-494-81549-6$
Our file Notre référence
ISBN: $978-0-494-81549-6$
NOTICE:

The author has granted a nonexclusive license allowing Library and Archives Canada to reproduce, publish, archive, preserve, conserve, communicate to the public by telecommunication or on the Internet, loan, distribute and sell theses worldwide, for commercial or noncommercial purposes, in microform, paper, electronic and/or any other formats.

The author retains copyright ownership and moral rights in this thesis. Neither the thesis nor substantial extracts from it may be printed or otherwise reproduced without the author's permission.
AVIS:

L'auteur a accordé une licence non exclusive permettant à la Bibliothèque et Archives Canada de reproduire, publier, archiver, sauvegarder, conserver, transmettre au public par télécommunication ou par l'Internet, prêter, distribuer et vendre des thèses partout dans le monde, à des fins commerciales ou autres, sur support microforme, papier, électronique et/ou autres formats.

L'auteur conserve la propriété du droit d'auteur et des droits moraux qui protège cette thèse. $\mathrm{Ni}$ la thèse ni des extraits substantiels de celle-ci ne doivent être imprimés ou autrement reproduits sans son autorisation.
In compliance with the Canadian Privacy Act some supporting forms may have been removed from this thesis.

While these forms may be included in the document page count, their removal does not represent any loss of content from the thesis.
Conformément à la loi canadienne sur la protection de la vie privée, quelques formulaires secondaires ont été enlevés de cette thèse.

Bien que ces formulaires aient inclus dans la pagination, il n'y aura aucun contenu manquant.

\section{Canadä}


The undersigned recommend to

the Faculty of Graduate and Postdoctoral Affairs

acceptance of the thesis

\title{
Interference from Large Wireless Networks under Correlated Shadowing
}

\author{
submitted by
}

Sebastian S. Szyszkowicz, M.A.Sc., B.A.Sc.

in partial fulfilment of the requirements for

the degree of Doctor of Philosophy in Electrical and Computer Engineering

Chair, Howard Schwartz, Department of Systems and Computer Engineering

Thesis Supervisor, Halim Yanıkömeroḡlu

External Examiner, Jacek Iłów, Department of Electrical and Computer

Engineering, Dalhousie University, Halifax, Canada

Carleton University

January 2011 


\section{Abstract}

We study the statistical distribution of the total interference power caused by a large wireless network and aggregated at a single victim receiver. We show that correlation between shadowing paths becomes a dominating factor in the interference from large networks, and should not be neglected. We thus propose a new problem formulation, which may include any pathloss, interferer distribution, and shadowing spread and correlation model. We make an extensive study of the existing correlation models for shadowing, and identify one that has the most desirable mathematical and physical characteristics. We then formulate a two-fold solution to the problem of finding the total interference distribution. We first develop an analytically-tractable approximation that applies to a subset of the interference scenarios. In order to solve the problem more generally, we then propose a reformulation in three steps of the simulation algorithm that achieve time gains of the order of 1000 , with minimal loss in accuracy. These two approaches allow us to solve a highly computationally intensive problem in seconds, not hours, on a personal computer. The algorithms are validated by via direct Monte Carlo simulations.

The problem is of great interest for enabling aggressive channel reuse and heterogeneous network coexistence in future wireless systems, where interference is expected to be the dominating factor. 


\section{Acknowledgements}

To my supervisor and hocam ${ }^{1}$, Dr. Halim Yanıkömeroğlu, for his endless support, patience, encouragement, and indeed friendship. I could not have wished for a better supervisor.

My sincere gratitude goes towards my thesis examiners:

- Prof. Paul C. Johns (Committee Chair), Dept. Physics, Carleton University,

- Prof. Jacek Iłów (External Examiner), Dept. Electrical and Computer Engineering, Dalhousie University, Halifax,

- Prof. Yiqiang Zhao, School of Mathematics and Statistics, Carleton University,

- Prof. Abbas Yongaçoglu, School of Information Technology and Engineering, University of Ottawa,

- Prof. Ioannis Lambadaris, Dept. Systems and Computer Engineering, Carleton University,

for carefully reviewing my work and giving me valuable comments towards the improvement of this thesis, as well as for a very pleasant defence experience.

Thank you to my colleagues in our group and department for the technical discussions as well as the lighter moments. You have made my time at Carleton both enjoyable and instructive. My thanks in particular to Furkan Alaca and Arshdeep

\footnotetext{
${ }^{1}$ hocam: "my teacher", honorific (Turkish)
} 
S. Kahlon, whom I have had the pleasure to mentor and work with, and who have taught me a great deal in their turn.

My thanks go to the many researchers with whom I had the honour to interact during the course of my graduate studies, for their hospitality and warmth during my travel visits, and for many interesting and enlightening technical discussions, which deepened my understanding of many topics, and helped sharpen several ideas in this thesis. Most notably, I would like to thank Prof. John S. Thompson, University of Edinburgh, Scotland, UK, for his generosity and friendliness in hosting me as a visiting researcher in the winter of 2008 , and from a valuable research collaboration therefrom.

I would also like to thank the ladies of our department office, for their generous and courteous help in navigating the administrative aspects of academic life. My gratitude also goes towards the gentlemen in our technical staff for their competent and patient help with all my computing needs. My thanks also go to the members of the cleaning and support staff here in the Minto CASE building, for providing me with a clean and functional working environment.

To my good friends far and near, you who make life light to bear, you who know the good and the bad of me $\mathrm{e}^{2}$. Thank you for being there for me through thick and thin, for sharing in my joys and sorrows, for rejoicing in my successes and for helping me recover from my failures. My thanks in particular go to Nick Milne, for his courageous proofreading of this entire thesis.

Thank you to my parents, Barbara and Mieczysław, for the gift of life, for their many sacrifices for me, for teaching me many of the most important things. A big hug to my little sister Kasia for always being there to cheer me up and for preventing me from taking myself too seriously. You make me very proud!

\footnotetext{
2 "Friends are those with whom our faults are safe." - G. K. Chesterton
} 
In the beginning was the Word, and the Word was with God, and the Word was God. [...] And the Word was made flesh, and dwelt among us.

— The Gospel of John 


\section{Contents}

$\begin{array}{ll}\text { Abstract } & \text { iii }\end{array}$

Acknowledgements $\quad$ iv

Contents $\quad$ vii

List of Figures $\quad$ xiii

List of Tables $\quad$ xvi

Nomenclature $\quad$ xvii

1 Introduction $\quad 1$

1.1 Motivation for this Research . . . . . . . . . . . . . . . 2

1.2 Positioning our Work with Respect to Current Literature . . . . . . . 4

1.2.1 Sums of Lognormal Random Variables . . . . . . . . . . . . . 4

1.2.2 Interference from Poisson Fields . . . . . . . . . . . . . . 6

1.2.3 Correlated Shadowing through Shadowing Fields . . . . . . . . 7

1.2.4 Our Work as a Synthesis of these Lines of Research . . . . . . 8

1.3 Plan of the Thesis . . . . . . . . . . . . . . 10

1.4 Mathematical Tools . . . . . . . . . . . . . . . . . 12

1.4 .1 Linear Algebra . . . . . . . . . . . . . . 12

1.4.2 Gaussian Random Variables and Processes . . . . . . . . . . . 13 
1.4.3 Convergence of Random Variables . . . . . . . . . . . . . . 15

1.4.4 Exchangeable Random Variables . . . . . . . . . . 16

2 Choosing a Good Shadowing Correlation Model 18

2.1 Types of Correlation . . . . . . . . . . . . . . . . . 20

2.1 .1 Scenarios . . . . . . . . . . . . . . . 20

2.1.2 Auto-Correlation . . . . . . . . . . . . . . . . 21

2.1 .3 Cross-Correlation . . . . . . . . . . . . . . . . . . 22

2.1.4 Mathematical Synthesis of Auto- and Cross-Correlation . . . 22

2.1.5 Generalised Correlation . . . . . . . . . . . . . . 23

2.1.6 Time-Correlation . . . . . . . . . . . . . . . . . 24

2.1.7 Uplink-Downlink Correlation . . . . . . . . . . . . . . . . 24

2.2 Positive Semidefiniteness of Correlation Models . . . . . . . . . 25

2.2.1 Models with Variable Shadowing Variance . . . . . . . 25

2.2.2 Methods for Proving Positive Semidefiniteness . . . . . . . . 26

2.2.3 One-Parameter and Separable Correlation . . . . . . . . 27

2.2.4 Incorporating Time and Uplink-Downlink Correlation . . . . 28

2.3 Estimating the Correlation Function from Measured Data . . . . . . . 31

2.3.1 Auto-Correlation as Mixed Time-Space Measurements . . . . 31

2.3.2 Cross-Correlation as Spatial-Only Correlation . . . . . . . . 32

2.3.3 Collapsing Correlation onto One Dimension . . . . . . . . 33

2.4 Specific Correlation Models and Their Positive Semidefiniteness . . . 34

2.4 .1 Constant Model . . . . . . . . . . . . . . . . . . . . 34

2.4.2 Absolute Distance-Only Models . . . . . . . . . . . . . 35

2.4.2.1 Exponential Model . . . . . . . . . . . . . 36

2.4.2.2 More Complex Models . . . . . . . . . . . . . . 37

2.4 .3 Angle-Only Models . . . . . . . . . . . . . . . . . 38 
2.4 .4 Separable Models . . . . . . . . . . . . . . . . . . . 40

2.4.4.1 Angle-Distance Ratio . . . . . . . . . . . . . . 41

2.4.4.2 Angle-Absolute Distance . . . . . . . . . . . 42

2.4.5 More Elaborate (Non-Separable) Models . . . . . . . . . . . 43

2.5 Some Thoughts on Physical Realism . . . . . . . . . . . . 45

2.6 Feasibility According to Marginal Distribution . . . . . . . . . . . 49

2.6.1 Jointly Lognormal Shadowing . . . . . . . . . . . . . . . . 49

2.6.2 Non-Lognormal Shadowing . . . . . . . . . . . . . 49

2.7 Consequences for Working with Correlated Shadowing . . . . . . . 50

2.7.1 For Those Wanting to Incorporate Shadowing Correlation . . 51

2.7.2 For Those Using a Model that Is Not Feasible . . . . . . . . 51

2.7.3 For Those Designing New Correlation Models . . . . . . . . . 51

2.7.4 For Those Studying Large Interference Problems . . . . . . . . 52

3 Problem Statement $\quad 53$

3.1 Interferer Layout $\ldots \ldots \ldots \ldots \ldots$

3.1 .1 Poisson Field . . . . . . . . . . . . . . . . 53

3.1 .2 Weighed Spatial Distribution . . . . . . . . . 54

3.1 .3 Cluster Layouts . . . . . . . . . . . . . . . . . . 55

3.1 .4 Layout Examples . . . . . . . . . . . . . . . . . 55

3.2 Channel Models . . . . . . . . . . . . . . . . . . . . . 57

3.2 .1 Average Pathloss . . . . . . . . . . . . . . . . 57

$3.2 .2 \quad$ Lognormal Shadowing . . . . . . . . . . . . . . 58

3.2.3 Spatially-Correlated Jointly Lognormal Shadowing . . . . . 58

3.3 Interferer Transmission . . . . . . . . . . . . . . . . . 60

3.4 Total Interference . . . . . . . . . . . . . . . . . . 60 
4 Analytical Results on the Aggregate Interference 63

4.1 Approximate Solution using Exchangeability . . . . . . . . . . 63

4.1.1 Limit Theorem on the Sum of Exchangeable Joint Lognormals 64

4.1.1.1 Problem Formulation . . . . . . . . . . 64

4.1.1.2 Main Result and Proof . . . . . . . . . . 65

4.1.1.3 Lognormal Approximation for Large $N \ldots 66$

4.1.2 Lognormal Approximation to Total Interference Distribution . 67

4.1.2.1 Comparing the Two Problems . . . . . . . . 67

4.1.2.2 Cross-Moment-Matching between the Two Problems 70

4.1.2.3 Simple Semi-Analytical Solution . . . . . . . . 71

4.2 Study of Moments ................... 73

4.2.1 Formulation of Moments . . . . . . . . . . . . 73

4.2 .2 Numerical Evaluation of Moments . . . . . . . . . . . . . 74

4.2.3 Using Moments in the Large- $N$ Regime . . . . . . . . . . 75

4.2.3.1 Mean-Matching............ 76

4.2.3.2 Variance-Matching . . . . . . . . 76

4.2.3.3 Matching both Mean and Variance . . . . . . . 77

4.3 Problem Reformulation Using a Stochastic Integral . . . . . . . . . 78

4.3.1 Equivalent Stochastic Integral for $I / N$ as $N \rightarrow \infty \ldots 79$

4.3.2 Mathematical Argument . . . . . . . . . . . . 79

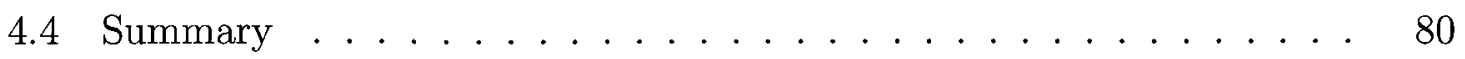

5 Simulation Results and Optimisation $\quad 81$

5.1 Simulation Setup ..................... 81

5.1.1 Simulation Platform ............... 81

5.1.2 Traditional Approach: Matrix Factorisation . . . . . . . . 82

5.1.3 Representing the Simulation Results . . . . . . . . . . 84 
5.1.4 Correlated versus Independent Shadowing . . . . . . . . 86

5.2 Evaluating the Accuracy of the Lognormal Approximation Method . 86

5.2.1 Accuracy of Convergence of the SEJLN Distribution to the Lognormal . . . . . . . . . . . . . . . 86

5.2.2 Numerical Evaluation of the Geometric Coefficients . . . . . 94

5.2.3 Lognormal Approximation to $I$ in a Clustered Layout . . . . 96

5.2.4 Lognormal Approximation to $I$ in a Non-Clustered Layout . . 96

5.3 Simulating I Rapidly: Combined Simulation-Numerical-Analytical Approach . . . . . . . . . . . . . . . . 103

5.3 .1 Shadowing Fields . . . . . . . . . . . . . . . . . 105

5.3.2 Efficient Filtering for Separable Triangular Correlations . . . . 109

5.3.2.1 Separability . . . . . . . . . . . . . . . 109

5.3.2.2 Optimised Box Filters . . . . . . . . . . . . . 109

5.3.2.3 Optimised Shadow Fields Algorithm _. . . . . . . 109

5.3 .3 Reusing Random Samples . . . . . . . . . . . . . . . . 111

5.3.3.1 Reuse in Matrix Factorisation . . . . . . . . . . 112

5.3.3.2 Reuse in Shadowing Fields . . . . . . . . . . . . 113

5.3 .4 Simulator Calibration . . . . . . . . . . . . . . . 114

5.3.5 Time Performance Comparison _. . . . . . . . . . . . 119

5.3.6 Moment-Corrected Extrapolations for High $N \ldots \ldots$. . . . 121

5.3.7 Optimising Other Correlation Models . . . . . . . . . 127

6 Conclusion and Future Work 128

6.1 Achievements of this Thesis . . . . . . . . . . . . . . 128

6.2 Immediate Model Extensions . . . . . . . . . . . . . . . . . . . 131

6.2 .1 Random Number of Interferers . . . . . . . . . . . . . 131

6.2.2 Non-Independent Interferer Positions . . . . . . . . . . . . 133 
6.2.3 Small-Scale Fading and Variable Interferer Transmit Power . . 133

6.2.4 Directional Victim Receiver Antenna . . . . . . . . . . . 135

6.2.5 Other Dimensions: Time and Frequency . . . . . . . . 136

6.3 Long-Term Research Questions . . . . . . . . . . . . . 137

$\begin{array}{ll}\text { Bibliography } & 140\end{array}$ 


\section{List of Figures}

1.1 Plan of thesis argument, with corresponding sections and publications. 10

2.1 a) Shadowing auto-correlation for a mobile $Y(t)$, b) Shadowing crosscorrelation for a mobile $X \ldots \ldots \ldots \ldots \ldots \ldots$

2.2 Generic geometry of shadowing auto- and cross-correlation. . . . . 23

2.3 A pair of auto- or cross-correlated paths, with the most relevant dimensional variables: $d, \theta, R \ldots \ldots \ldots \ldots$

2.4 Shadowing correlation model families according to physical dimensions described in Figure 2.3. Other parameter combinations are possible but have not been encountered in literature. . . . . . . . . . . .

2.5 Physical argument of common propagation area for correlation in shad-

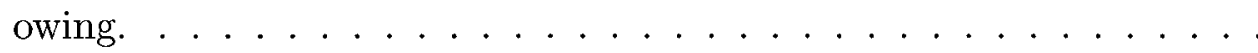

3.1 Interferer layouts used in this thesis. Distances are in meters. . . . . 56

3.2 Shadowing correlation function (3.5) with $\theta_{0}=60^{\circ}$ and $R_{0}=6 \mathrm{~dB}$ : the correlation coefficient between two shadowed paths beginning at base of the arrow. One path ends at the tip of the arrow, while the other is located anywhere on the plane. The figure is invariant under rotation and scaling. . . . . . . . . . . . . .

5.1 Interference cdfs on lognormal paper: with and without shadowing

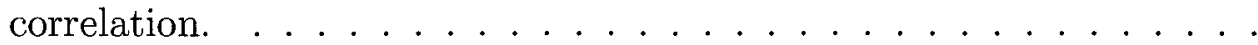


5.2 SEJLN cdf on lognormal paper, $\sigma=6 \mathrm{~dB}, \rho=0.5$, with varying $N . \quad 88$

5.3 SEJLN cdf on lognormal paper, $\sigma=6 \mathrm{~dB}, \rho=0.05$, with varying $N . \quad 89$

5.4 SEJLN cdf on lognormal paper, $\sigma=6 \mathrm{~dB}, \rho=0.005$, with varying $N . \quad 90$

5.5 SEJLN cdf on lognormal paper, $\sigma=12 \mathrm{~dB}, \rho=0.5$, with varying $N . \quad 91$

5.6 SEJLN cdf on lognormal paper, $\sigma=12 \mathrm{~dB}, \rho=0.05$, with varying $N . \quad 92$

5.7 SEJLN cdf on lognormal paper, $\sigma=12 \mathrm{~dB}, \rho=0.005$, with varying $N .93$

5.8 Total interference cdf on lognormal paper for Layout A, with $\sigma_{\mathrm{s}}=6 \mathrm{~dB}$, $\beta=4$, and $N=1,10,100,1000$ from left to right. . . . . . . . 97

5.9 Total interference cdf on lognormal paper for Layout $\mathrm{A}$, with $\sigma_{\mathrm{s}}=$ $12 \mathrm{~dB}, \beta=3$, and $N=1,10,100,1000$ from left to right. . . . . . . 98

5.10 Total interference cdf on lognormal paper for Layout $\mathrm{B}$, with $\sigma_{\mathrm{s}}=6 \mathrm{~dB}$, $\beta=3$, and $N=1,10,100,1000$ from left to right. . . . . . . 99

5.11 Total interference cdf on lognormal paper for Layout $\mathrm{B}$, with $\sigma_{\mathrm{s}}=$ $12 \mathrm{~dB}, \beta=4$, and $N=1,10,100,1000$ from left to right. . . . . . 100

5.12 Total interference cdf on lognormal paper for Layout C, with $\sigma_{\mathrm{s}}=6 \mathrm{~dB}$, $\beta=4$, and $N=1,10,100,1000$ from left to right.

5.13 Total interference cdf on lognormal paper for Layout $\mathrm{D}$, with $\sigma_{\mathrm{s}}=6 \mathrm{~dB}$, $\beta=4$, and $N=1,10,100,1000$ from left to right. . . . . . . 102

5.14 Shadowing field realisations for $r_{\min }=50, r_{\max }=500, \theta_{0}=60^{\circ}, R_{0}=$ $6 \mathrm{~dB}$, with increasing resolution: $D_{\Theta}=6 n, D_{\mathrm{R}}=5 n, F_{\Theta}=n, F_{\mathrm{R}}=3 n$, where a) $n=1$, b) $n=2$, c) $n=5$, d) $n=50$. The colour of the areas corresponds to the value of $S_{\imath} / \sigma_{\mathrm{s}}\left(\vec{r}_{2}\right)$ therein. . . . . . . . 106

5.15 Calibration of field resolution parameters $D_{\Theta}=6 n$ and $D_{\mathbf{R}}=5 n$, and the resulting simulated cdfs of $I \ldots \ldots \ldots \ldots$

5.16 Effect of random sample reuse in matrix factorisation algorithm on the cdf of $I . K=1000000, K_{\vec{r}}=K_{\mathrm{Ch}}=K / m \ldots \ldots \ldots \ldots$ 
5.17 Effect of combining random sample reuse with calibrated $\left(D_{\ominus}=12, D_{\mathrm{R}}=\right.$ 10) shadowing fields algorithm on the cdf of $I . K=1000000, K_{\vec{r}}=$ $K_{\mathrm{Ch}}=K / m \ldots \ldots \ldots \ldots \ldots \ldots \ldots \ldots \ldots \ldots \ldots \ldots \ldots \ldots$

5.18 Execution time performance of matrix (Cholesky) factorisation versus shadowing fields, and of no reuse versus random sample reuse. Simulation parameters are given in Table 5.2. . . . . . . . . . 120

5.19 Using mean-matching (4.24) to extrapolate the cdf of $I$ for very high $N$. All simulations are done using shadowing fields with reuse. . . . . 123

5.20 Using variance-matching (4.26) to extrapolate the cdf of $I$ for very high $N$. All simulations are done using shadowing fields with reuse. . 124

5.21 Using two-moment-matching (4.31) to extrapolate the cdf of $I$ for very high $N$. All simulations are done using shadowing fields with reuse. . 125 


\section{List of Tables}

2.1 Summary of existing shadowing correlation models and their properties. 46

5.1 Geometric coefficients for each layout in Figure 3.1. . . . . . . . . . 94

5.2 Simulation settings after calibration. . . . . . . . . . . . . . 104 


\section{Nomenclature}

\section{Acronyms}

\begin{tabular}{ll}
\hline Acronym & Meaning \\
\hline \hline cdf & cumulative distribution function \\
CLT & central limit theorem \\
FFT & fast Fourier transform \\
iid & independent and identically distributed \\
IS & interfering station \\
LLN & law of large numbers \\
pdf & probability density function \\
PPP & Poisson point process \\
psd & positive semidefinite \\
RP & random process \\
RV & random variable \\
RX & victim receiver station \\
SEJLN & sum of exchangeable joint lognormals \\
\hline
\end{tabular}




\section{Mathematical Operators and Symbols}

\begin{tabular}{|c|c|}
\hline Notation & Meaning \\
\hline * & convolution \\
\hline$\left[X_{\imath}\right]_{\imath=1}^{N}$ & vector of elements $X_{1}, X_{2}, \ldots, X_{N}$ \\
\hline$\left\{X_{\imath}\right\}_{\imath=1}^{N}$ & set of elements $X_{1}, X_{2}, \ldots, X_{N}$ \\
\hline$|\mathcal{S}|$ & "natural" measure of set $\mathcal{S}$, e.g., area, volume, etc. \\
\hline $\mathcal{A} \backslash \mathcal{B}$ & set subtraction: the set of elements in $\mathcal{A}$ not in $\mathcal{B}$ \\
\hline $\mathcal{C}=\mathcal{A} \times \mathcal{B}$ & Cartesian product of sets: $x \in \mathcal{A}, y \in \mathcal{B} \Rightarrow(x, y) \in \mathcal{C}$ \\
\hline$f: \mathcal{A} \mapsto \mathcal{B}$ & $f$ is a mapping from the set $\mathcal{A}$ to the set $\mathcal{B}$. \\
\hline$f: x \mapsto y$ & $f$ maps $x$ to $y$ \\
\hline$X \stackrel{\mathcal{D}}{\longrightarrow} Y$ & $\mathrm{RV} X$ converges in distribution to $\mathrm{RV} Y$. \\
\hline$X \stackrel{\mathcal{D}}{=} Y$ & $\mathrm{RV} X$ and $\mathrm{RV} Y$ have the same distribution. \\
\hline$X \stackrel{\mathcal{D}}{\approx} Y$ & $\mathrm{RV} X$ and $\mathrm{RV} Y$ have approximately the same distribution. \\
\hline $\mathcal{A} \mid \mathcal{B}$ & statistical conditioning of $\mathcal{A}$ given $\mathcal{B}$ (of $\mathrm{RVs}$ or events) \\
\hline$\left[x_{\imath, 3}\right]_{M \times N}$ & $\begin{array}{l}\text { matrix composed of entries } x_{\imath, 3} \text { with indices } i=1, \ldots, M, j= \\
1, \ldots, N\end{array}$ \\
\hline $\mathbf{A}^{\mathrm{T}}$ & the matrix (or vector) transpose of $\mathbf{A}$ \\
\hline$\sqrt[*]{\mathrm{K}}$ & $\begin{array}{l}\text { any } N \times N \text { matrix decomposition of } N \times N \text { matrix } \mathbf{K} \text { such that } \\
\sqrt[*]{\mathbf{K}} \sqrt[*]{\mathbf{K}}^{\mathrm{T}}=\mathbf{K}\end{array}$ \\
\hline$\circ$ & Hadamard/Schur matrix product \\
\hline$\otimes$ & Kronecker/Zehfuss matrix product \\
\hline $\operatorname{diag}\left(x_{1}, \ldots, x_{N}\right)$ & $\begin{array}{l}\text { square matrix with entries } x_{1}, \ldots, x_{N} \text { on the main diagonal, and } \\
\text { zero elsewhere }\end{array}$ \\
\hline $\mathbb{E}\{X\}$ & expected value of $\mathrm{RV} X$ \\
\hline $\mathbb{N}^{*}$ & the set of natural numbers (excluding zero) \\
\hline $\mathcal{N}(\vec{\mu}, \mathbf{K})$ & $\begin{array}{l}\text { jointly-Gaussian (normal) distribution with mean vector } \vec{\mu} \text { and co- } \\
\text { variance matrix } \mathbf{K}\end{array}$ \\
\hline $\mathcal{O}(f(N))$ & order of $f(N)$, "Big-O" notation \\
\hline $\mathbb{P}(\mathcal{E})$ & probability of event $\mathcal{E}$ occurring \\
\hline$\Phi(x)$ & standard Gaussian cdf \\
\hline $\mathbb{R}$ & the set of real numbers \\
\hline
\end{tabular}




\begin{tabular}{ll}
$\mathbb{R}^{+}$ & the set of non-negative real numbers (including zero) \\
$R_{X}(\tau)$ & $\begin{array}{l}\text { autocorrelation function of stationary process } X(t) \text { (can be ex- } \\
\text { tended to random fields) }\end{array}$ \\
$\mathbb{V A R}\{X\}$ & variance of RV $X$ \\
$\mathbb{Z}$ & the set of integers \\
\hline
\end{tabular}




\section{Physical and Mathematical Quantities}

\begin{tabular}{ll}
\hline Symbol & Meaning \\
\hline \hline$A, B, C$ & moments and cross-moments of $I_{\imath}$ \\
$a, b, c, a^{\prime}, b^{\prime}, c^{\prime}$ & scaling coefficients for extrapolation to larger $N$ \\
$\mathcal{A}$ & square region in stochastic integral argument, that entirely contains \\
& $\mathcal{A}_{g}$ \\
$\mathcal{A}_{g}$ & entire region where $g$ is non-zero \\
$\mathcal{A}_{l}$ & sub-square region of $\mathcal{A}$ \\
$\beta$ & pathloss exponent when average pathloss is power-law \\
$\mathbf{C}$ & $N \times N$ matrix such that CC ${ }^{\mathrm{T}}=\mathbf{K}$, if it exists \\
$D_{\mathrm{R}}$ & length of discrete shadowing field in the $R$ dimension \\
$D_{\Theta}$ & length of discrete shadowing field in the $\theta$ dimension \\
$d$ & Cartesian distance between two points, used in shadowing correla- \\
& tion models \\
$d_{\mathrm{m}}$ & distance covered by a mobile at time $t$ when considering shadowing \\
$\delta$ & measurements \\
$\eta_{x}(\xi) \eta_{y}(v)$ & side length of square $\mathcal{A}$ \\
$\mathbf{F}$ & separable auto-correlation function of $\overline{\mathbf{M}}$ \\
$F_{\mathrm{R}}$ & discrete filter kernel for generating $\overline{\mathbf{M}}$, of size $F_{\mathrm{R}} \times F_{\Theta}$ \\
$F_{\Theta}$ & length of discrete shadowing field filter in the $R$ dimension \\
$g(\vec{r})$ & length of discrete shadowing field filter in the $\theta$ dimension \\
$\bar{g}(r)$ & two-dimensional pdf of IS locations $\vec{r}_{\imath}$ \\
$G_{1}, G_{2}$ & pdf of $r_{\imath}$ derived from $g(\vec{r})$ \\
$G_{\text {cor }}$ & constants obtained by integration over $g()$ \\
$\mathbf{H}$ & constant obtained by integration over $g()$ and $h()$ \\
$h\left(\vec{r}_{1}, \vec{r}_{2}\right)$ & correlation coefficient matrix of $\vec{S}$, formed by $\left.\left[h\left(\vec{r}_{1}, \vec{r}_{2}\right)\right]_{N \times N}, \vec{r}_{2}\right)$ \\
$\bar{h}\left(\vec{r}_{1}, \vec{r}_{2}\right)$ & shadowing correlation model \\
$\hat{h}\left(\vec{r}_{1}, \vec{r}_{2}\right)$ & true physical shadowing correlation function \\
$\tilde{h}\left(\vec{r}_{1}, \vec{r}_{2}\right)$ & stimated physical shadowing correlation function \\
& shadowing correlation model for the return paths of those of \\
&
\end{tabular}




\begin{tabular}{|c|c|}
\hline$h_{\mathrm{d}}(d)$ & correlation model along the $d$ dimension, when $h$ is thus separable \\
\hline$h_{\mathrm{R}}(R)$ & correlation model along the $R$ dimension, when $h$ is thus separable \\
\hline$h_{\Theta}(\theta)$ & correlation model along the $\theta$ dimension, when $h$ is thus separable \\
\hline$I$ & total interference power at the RX from all ISs \\
\hline$I_{\imath}$ & interference power at the RX from IS $\imath$ \\
\hline$J_{l}$ & binomial RV representing the number of points falling in square $\mathcal{A}_{l}$ \\
\hline$J_{0}(x)$ & Bessel function of the first kind \\
\hline $\mathbf{K}$ & covariance matrix of $\vec{S}$ \\
\hline$\overline{\mathbf{K}}$ & $\begin{array}{l}\text { expanded covariance matrix of shadowing, given points in time, } \\
\text { space, and both path directions }\end{array}$ \\
\hline$K$ & number of trials of $I$ generated in Monte Carlo simulations \\
\hline$K_{\mathrm{Ch}}$ & number of trials of $\vec{S}$ \\
\hline$K_{\vec{r}}$ & number of trials of $\left[\vec{r}_{\imath}\right]_{\imath=1}^{N}$ \\
\hline$\lambda$ & $\begin{array}{l}\text { constant equal to } 0.1 \ln 10 \approx 0.2303 \text {, used to convert between expo- } \\
\text { nentials and } \mathrm{dB}\end{array}$ \\
\hline$\lambda_{\mathrm{P}}$ & density of a Poisson point process \\
\hline $\mathbf{M}$ & discrete version of $\overline{\mathbf{M}}$ \\
\hline $\bar{M}$ & $\begin{array}{l}\text { continuous stationary field representing the shadowing field under } \\
\text { a geometric transformation }\end{array}$ \\
\hline$M$ & scaling factor for the number of ISs, used in extrapolation \\
\hline$m$ & reuse factor, defined when $K_{\mathrm{Ch}}=K_{\vec{r}}$ \\
\hline$m_{V}$ & mean parameter of lognormal RV approximating the SEJLN RV $V$ \\
\hline$m_{\infty}$ & mean parameter of limiting lognormal RV of $V / N$ as $N \rightarrow \infty$ \\
\hline$\mu$ & mean coefficient in the SEJLN distribution \\
\hline$N$ & number of ISs (a non-random value) \\
\hline$p(r)$ & average pathloss function \\
\hline$q_{l}$ & length of $\vec{q}_{l}$ \\
\hline $\overrightarrow{q_{l}}$ & position of centre of square $\mathcal{A}_{l}$ \\
\hline$R$ & distance ratio in $\mathrm{dB}$, used in shadowing correlation models \\
\hline$R_{0}$ & distance ratio parameter in correlation model (3.5) \\
\hline$R_{S}(\tau)$ & auto-correlation function of $S(t), S_{\imath}(t)$, and $\tilde{S}_{\imath}(t)$ \\
\hline
\end{tabular}




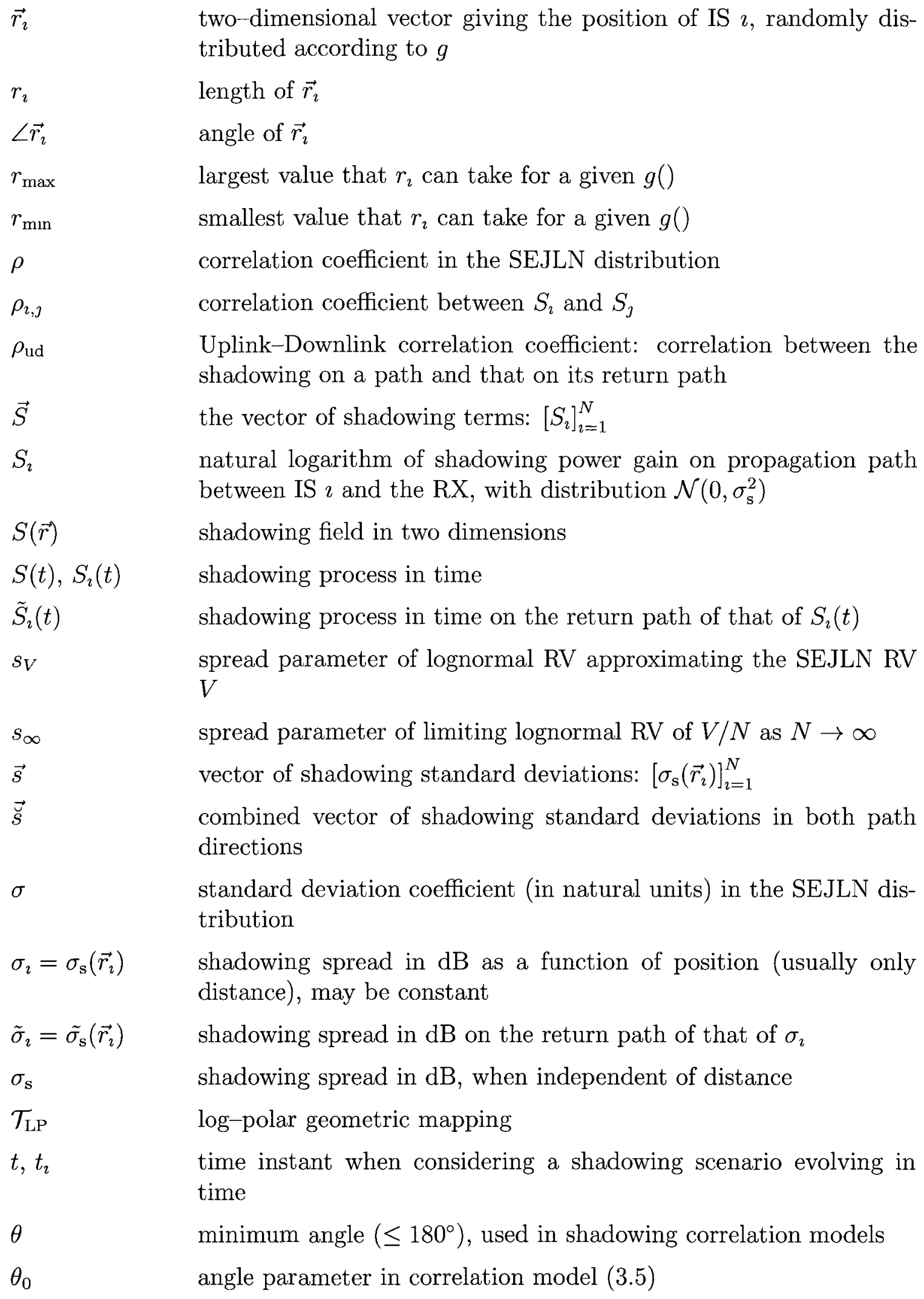




\begin{tabular}{|c|c|}
\hline$v$ & speed of a mobile, equal to $\|\vec{v}\|$ \\
\hline$\vec{v}$ & $\begin{array}{l}\text { velocity vector of a mobile when considering shadowing measure- } \\
\text { ments }\end{array}$ \\
\hline$V$ & SEJLN RV \\
\hline$V_{\imath}$ & exchangeable jointly Gaussian RVs \\
\hline$W$ & common shadowing component of $S_{1}$ and $S_{2}$ \\
\hline$W_{\imath}$ & shadowing component exclusively of $S_{\imath}$ \\
\hline$w^{2}, w_{1}^{2}$ and $w_{2}^{2}$ & variances of $W, W_{1}$, and $W_{2}$, respectively \\
\hline$X, X_{\imath}, Y, Y_{\imath}$ & endpoints of propagation paths \\
\hline $\overrightarrow{X Y}$ & a directed propagation path from point $X$ to point $Y$ \\
\hline$Z, Z_{\imath}$ & independent standard Gaussian RVs \\
\hline$\vec{Z}$ & vector of iid standard Gaussian RVs \\
\hline$\vec{z}$ & vector of the inverse of shadowing standard deviations: $\left[\sigma_{\mathrm{s}}^{-1}\left(\vec{r}_{\imath}\right)\right]_{\imath=1}^{N}$ \\
\hline$\zeta(\tau)$ & normalised auto-correlation function of $S(t), S_{\imath}(t)$, and $\tilde{S}_{\imath}(t)$ \\
\hline
\end{tabular}

Section 2.4 also describes a series of existing shadowing models whose parameter names are often taken directly from literature and should not be confused with the above notations. These parameters are only used in that section and in Table 2.1, and pertain only to the model in which they are used. These are: $A, a$ (multiple times), $\alpha$ (multiple times), $B, b$ (multiple times), $d_{0}, d_{1}, d_{2}, d_{\text {sep }}, \bar{d}, \gamma, K, \nu, \rho, \theta_{\mathrm{T}}$. 


\section{Chapter 1}

\section{Introduction}

This thesis is about aggregate interference power at a victim receiver, and about spatial correlation in wireless shadowing, two established lines of research in the wireless community. The purpose of this thesis is four-fold:

1. To demonstrate that shadowing correlation is indeed very important in large interference scenarios, and cannot be realistically ignored.

2. To develop a problem formulation that is a synthesis of many scenarios studied in literature: a problem that is realistic and general enough to cover the widest possible set of real interference scenarios, while still being tractable in some way.

3. To obtain the statistical distribution of this aggregate interference from a large number of interferers, in a manner that is accurate, computationally efficient, and easy to understand and implement in simulations.

4. To properly choose a shadowing correlation model and to implement it efficiently in a simulation platform.

The problem is of significant importance: the aggressive reuse of precious wireless spectrum needed to achieve futuristic wireless throughputs will require a very good theoretical understanding of heavy interference scenarios. 


\subsection{Motivation for this Research}

The number of wireless devices, both for personal use and for autonomous machine applications, is likely to grow in the near future. Indeed, if we include autonomous devices such as wireless sensors, there is the potential for even trillions of devices in the upcoming years [1]. The wireless spectrum, however, remains scarce, and an aggressive spatial frequency reuse will likely be necessary. Several recent works $[2,3]$ have taken an interest in practical interference scenarios, where the number of interferers is more than 100. The nature of the interfering nodes may be femtocells [2], sensor nodes, or any other devices that aggressively share spectrum, often in a noncoordinated and opportunistic manner. This type of scenario is becoming increasingly relevant as wireless communications move away from the traditional coordinated cellular model to more heterogeneous and distributed paradigms, such as ad-hoc networking and cognitive radio $[3,4]$. There is also potential for application in cellular systems that are augmented by a large number of autonomous devices, such as nomadic relays [5]. Thus the study of interference from many co-channel interferers is essential for the design of future wireless systems. The immediate implication for wireless networks will be heavy co-channel interference, particularly in denselypopulated (urban) scenarios, and perhaps in massively-deployed sensor networks as well. It is likely that many wireless systems will be interference-limited, with cochannel interference originating from very many wireless transmitters, and that the signal-to-interference ratio statistics will be an important performance metric. A good understanding of these interference scenarios will therefore be crucial in future wireless network planning.

It can be argued, however, that the state of literature on interference problems is not currently prepared to appropriately model these large numbers of interferers. On the side of analytical and numerical approaches, we have one of two situations: 
either very accurate results for small numbers of interferers, but that scale poorly for large networks; or analysis of large fields of wireless interferers, but this with very restrictive and simplistic channel and system models that may not at all correspond to most real scenarios. A synthesis of these two approaches is therefore in order. Several works [4,6-11] have considered large interfering networks with independent received interfering signals and concluded that the total received interference is approximately Gaussian, by application of the central limit theorem (CLT). The problem, however, is not so simple. Indeed, adding correlation produces very different results, as we show in this thesis, and therefore we propose our work as an improvement in accuracy over the CLT approach.

Furthermore, as computational power is increasing, we believe that the benefits of analysing small systems are waning, as most can be simulated via some Monte Carlo approach in good-enough time. This is not at all the case for large networks, where computational time and memory may become prohibitive. Such problems instead benefit from either an asymptotic analysis or a judicious reformulation of the problem that is less sensitive to the number of devices.

Finally, there exists a risk of a certain disjoint between theoretical results in the academic world, and results of practical value for vendors and network operators (this wide-ranging problem is addressed in $[1,12])$. We therefore emphasise the importance of developing results that are of practical use to designers: specifically, we want to provide a method that solves the problem at hand accurately, and with minimal human and computer resources. Apart from these goals, we want to solve the problem "by any means necessary", and we feel that a judicious mixture of analysis, numerical techniques, and Monte Carlo simulation might be the best solution to the problem at hand (this may be the case for many other problems also).

In this thesis, we follow these lines of thought to formulate

- a new problem that is very general, difficult and important to solve, while being 
useful in future interference scenarios,

- a set of solutions that benefit from accuracy, ease of implementation, and minimal computational effort.

\subsection{Positioning our Work with Respect to Current Literature}

The problem that we formulate and partly solve in this thesis has not been addressed before. Rather it lies at the intersection of three research arcs, of which the first two represent a significant portion of the work on wireless interference in recent years. We examine each sub-field in turn, note its strengths and weaknesses, and then explain how our work synthesises these approaches to produce a more realistic, general, and efficiently solvable problem.

\subsubsection{Sums of Lognormal Random Variables}

The problem of finding the distribution of the sum of several lognormal random variables (RVs) is a long-standing mathematical problem in the wireless community [13], and has received renewed interest since around 2004. We made a study of the proposed solutions in our Masters thesis [14] concerning pre-2007 literature, and produced some results of our own $[15,16]$. Since then, there have been significant advances in this field, notably [17-23].

The sum of lognormals approach is based on the idea that the interference arriving at the victim receiver station (RX) from any one interfering station (IS) is (approximately) lognormally distributed, given a significant shadowing component, a lesser (or ignored) effect from small-scale fading, and little or no statistical variability of the positions (which, through pathloss, affect the log-mean of the lognormal distributions). Furthermore, several of the new methods consider correlation between shadowing paths $[20,23]$ (as well as $[17,21]$, which only study the tail behaviour). 
This however requires that the lognormal terms be jointly lognormal, and further that the correlation coefficient of each pair of terms be known and fixed: this again implies statistically fixed IS positions.

This approach presents several inherent limitations:

1. The problem has proved very difficult to tract analytically. In fact, to this day, there is no exact closed form result for the distribution of even two independent and identically distributed (iid) lognormal RVs. Some very accurate approximations have been proposed in the last three years $[19,20,22,23]$. They are, however, quite complex, as can be seen from the lengths of the algorithms in these papers: they require a significant investment of human time to understand and implement numerically.

2. Because of the previous point, together with the rapidly increasing computation power available, for a small number of ISs it might be much more efficient to obtain the distribution of the sum of lognormals simply by Monte Carlo simulations, eschewing any analysis. This is amplified by the complexity of the aforementioned algorithms, which means that there is no obvious relation between the input parameters and the output: one simply obtains a curve, which could be equivalently obtained through simulation with minimal human effort and with well-controlled accuracy. The advantages of an analytical solution therefore fade away.

3. The analysis of the interference from a large number of ISs therefore becomes more advantageous, since simulations may then become prohibitively expensive. The implementation of the algorithms in $[20,23]$ may then become a more effective use of one's time. There are, however, several difficulties with this:

(a) The methods have not been tested for more than $N=20$ terms. One would 
need to study the accuracy and numerical stability of these methods for much larger $N$.

(b) The complexity and computational time required may grow significantly. Notably, the methods in $[19,20,23]$ are $\mathcal{O}\left(e^{N}\right)$. It is not immediately obvious how to circumvent this.

(c) It would be preferable to have some kind of asymptotic approach to a problem where there are many similar terms. But all these approaches explicitly need as input the parameters and correlation coefficients of all the lognormal RV. There is no obvious approach to simplify the problem for a large $N$.

4. The sum of lognormals is only an intermediate point in the analysis of interference, and even if this problem is solved efficiently and exactly, the interference problem is not thereby solved, at least not for large networks. Indeed, in large networks we do not expect to know the locations of the ISs explicitly, only statistically. The sum of lognormals only solves the problem for fixed joint statistics of the lognormal RVs, but when the locations are random, the means, correlation coefficients, and potentially even the variances of the underlying Gaussian RVs are themselves random, and the problem is more involved.

\subsubsection{Interference from Poisson Fields}

A complementary approach to the previous one, the problem of finding the interference from a Poisson field of ISs, has also received much interest since around $1990[4,11,24-35]$.

This approach also presents several inherent limitations, the main two being:

1. The statistical layout of the IS is very restrictive. The ISs are simply placed 
according to a uniform Poisson field over an infinite area, with possibly a protection radius (or "guard zone" [30]) around the RX (to avoid the problem of having transmitters arbitrarily close to the RX), as well as a limit radius to bound the area of interest (which also makes simulations possible). These works thus remain focused on annular sectors centred at the RX, with uniformly distributed ISs within. We feel this is a important limitation as to the applicability of the model. Furthermore, observing these works, we conclude that the analysis is heavily contingent on the geometry of the layout, and there does not seem to be an approach to abstract from that layout. This leads to the solving of many special cases without a unified approach. It is only recently that there has been interest in more generalised IS layouts [31,32, 34-36].

2. To the best of our knowledge, correlation in shadowing is not considered. However, as we show in this thesis, correlation between shadowing paths becomes a very important parameter when the number of interferers is large (as is naturally the case in Poisson field modeling). As such, the omission of correlation may be a serious deficiency in the accuracy of the results with respect to reality.

\subsubsection{Correlated Shadowing through Shadowing Fields}

Shadowing fields [37-42] are a computational method for generating correlated shadowing. They are not inherently bound to interference, and in fact we may be the first to make this application. While shadowing fields are indeed helpful to solving our problem, the existing literature presents some difficulties to be overcome:

1. The correlation model most often used in these works [43] has several deficiencies:

(a) As we argue in Chapter 2, it is of questionable realism, and it has only one tunable parameter. 
(b) It is difficult to simulate accurately, and some approaches [40] result in significant artifacts due to approximation that do not decrease with the field resolution.

2. The computational cost of generating field realisations may be quite high.

Shadowing fields should not be confused with shadowing maps [44-47], which are a similar but different approach that we do not use here.

\subsubsection{Our Work as a Synthesis of these Lines of Research}

Our work lies at the intersection of these three lines of research, and attempts a synthesis that overcomes the abovementioned deficiencies.

First, our approach overcomes the model limitations of the above three fields.

1. Our work includes correlation between shadowing paths. In fact, our approach works for any (reasonable, see Chapter 2) correlation model. The exception to this is in the efficient simulation of shadowing fields, where we exploit the particular properties of the model we use, though here again other models may also be used along the same lines, with perhaps slightly more modest gains in computation time.

2. Furthermore, we use a correlation model that has been chosen among many others for its particular advantages:

(a) It has, we argue, the best mathematical and physical properties from among the models in literature.

(b) It has two tunable parameters, which allow for a more general model. Additionally, one can approximate many other models by this one, as done in [48]. 
(c) We show that the model leads to a particularly efficient implementation of shadowing fields, which can be made arbitrarily accurate by increasing the field resolution.

3. Our approach considers randomly located ISs, and is applicable to any statistical layout of ISs as long as the area considered is not infinite and the positions are iid.

We also develop two approximate solutions using a mixture of elements from mathematical analysis, numerical integration, and Monte Carlo simulation. Our solutions also overcome many of the limitations of the previous works:

1. For an interesting subset of the problem, we provide a semi-analytical solution that has several important advantages:

(a) It is very simple (perhaps as simple as possible), and can be written in a few short equations. Its complexity does not depend at all on $N$. It can thus be applied to an interference problem with minimal time investment.

(b) It requires only some simple numerical integrals that can be computed in minimal time, and depend only on the layout and correlation model. The rest of the solution can be executed on a scientific calculator.

(c) The solution clearly separates the effects of the different parameters (layout, correlation model, number of ISs, pathloss exponent, shadowing spread), giving useful insight on the effect of each one.

(d) Because the method is based on a probability limit theorem, the accuracy actually improves as the number of ISs increases.

2. While the simulation cost of our model is very high, between $\mathcal{O}\left(N^{2}\right)$ and $\mathcal{O}\left(N^{3}\right)$, and $N$ may indeed be very large, we develop three successive approximations 
that cumulatively reduce the simulation cost to $\mathcal{O}(N)$, then $\mathcal{O}(1)$ for high enough $N$. In the end, we show how, in a typical example, we may simulate the interference distribution with good accuracy in 16 seconds for any $N$, compared to 10.5 hours for the classical approach when $N=1000$, on a personal computer (see Section 5.1.1 for specifications).

\subsection{Plan of the Thesis}

The structure of the argument presented in this thesis is illustrated in Figure 1.1. The thesis is organised as follows:

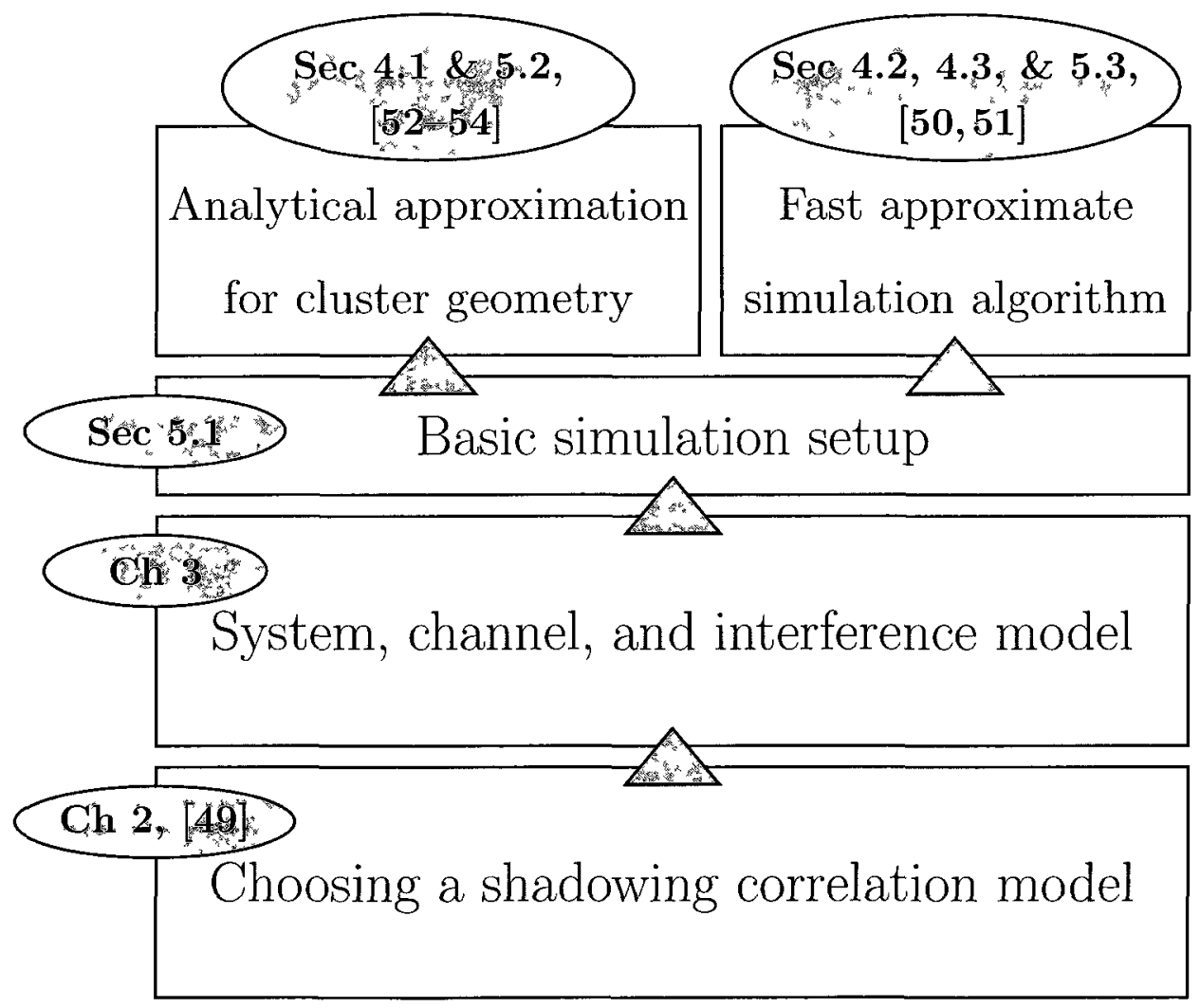

Figure 1.1: Plan of thesis argument, with corresponding sections and publications.

Chapter 2 examines the question of shadowing correlation in detail, and makes a thorough comparison of the existing models according to mathematical and physical 
criteria. The purpose of this chapter is twofold: firstly, it justifies the choice of a particular correlation model, which we use throughout the thesis (though most results can be evaluated for any correlation model); secondly, we propose it as a guide to researchers on how to choose, use, and design shadowing correlation models. The work in this chapter is published in [49].

Chapter 3 describes the wireless channel and system to be studied. This leads to the description of the aggregate interference power $I$. Finding the distribution of $I$ is the principal goal of this thesis.

Chapter 4 contains the analytical results developed in this thesis. Section 4.1 shows a simple approximation to the distribution of the total interference, a result that appears in [53], and is in preparation for a journal submission [54]. Our method is based on a limit theorem we developed for this very purpose, published as [52]. Section 4.2 considers the moments of the total interference and their behaviour when the network becomes large. Section 4.3 proposes a reformulation of the problem as a stochastic integral. While this integral is not solved, it provides useful insight that is later used to simulate correlated shadowing faster. These results appear in $[50,51]$.

Chapter 5 serves two purposes: firstly, in Section 5.2, we evaluate the accuracy of the analytical approximation developed in Section 4.1, and conclude that it performs well in clustered IS layouts only (which usually correspond to high average correlation). Most of these results appear in [52-54]. We then propose a general solution in Section 5.3, which uses the analytical and numerical results from Sections 4.2 and 4.3, along with several simulation techniques, to obtain fast, accurate approximations to the total interference distribution. This approach is validated via the slow standard simulation algorithm and provides simulation time gains of a factor of over 1000 for large networks. These methods and results are described in $[50,51]$.

Chapter 6 summarises the achievements of this thesis and proposes several axes for its extension, as well as more general perspectives for long-term research. 


\subsection{Mathematical Tools}

This work makes use of mathematical analysis, mostly in the realm of probability theory and linear algebra, and therefore requires a number of mathematical results and definitions, which we describe here.

\subsubsection{Linear Algebra}

Concepts of linear algebra are required in our work, notably in the proofs in Chapter 2. All algebraic operations are taken over the $\mathbb{R}$ field.

Definition 1. A square matrix $\mathbf{A}_{N \times N}$ is positive definite if for every column vector $\mathbf{x} \in \mathbb{R}^{N} \backslash \mathbf{0}, \vec{x}^{\mathrm{T}} \mathbf{A} \vec{x}>0$ [55, p.82 Def. 3.1.1.xvii].

Definition 2. A square matrix $\mathbf{A}_{N \times N}$ is positive semidefinite (psd) if for every column vector $\vec{x} \in \mathbb{R}^{N}, \vec{x}^{\mathrm{T}} \mathbf{A} \vec{x} \geq 0$ [55, p.82 Def. 3.1.1.xv].

Definition 3. The Hadamard (also called Schur) product of two $M \times N$ matrices A with entries $a_{\imath, \jmath}$ and $\mathbf{B}$ with entries $b_{\imath, \jmath}$, is a $M \times N$ matrix $\mathbf{C}=\mathbf{A} \circ \mathbf{B}$ with entries $c_{\imath, \jmath}$, such that the matrix entries are multiplied element-wise: $c_{\imath, \jmath}=a_{\imath, \jmath} b_{\imath, \jmath}[55$, p.252 Eq. 7.3.1].

Property 1 (Schur Product Theorem). If two $N \times N$ matrices $\mathbf{A}$ and $\mathbf{B}$ are both psd, then their Hadamard product $\mathbf{C}=\mathbf{A} \circ \mathbf{B}$ is also psd [55, p.335 Fact 8.16.8].

Definition 4. The Kronecker (also called Zehfuss) product of a $M \times N$ matrix A with entries $a_{\imath, \jmath}$ and a $K \times L$ matrix $\mathbf{B}$, is a $M K \times N L$ matrix $\mathbf{C}=\mathbf{A} \otimes \mathbf{B}$ with $M \times N$ block entries $a_{\imath, j}$ B [55, p.248 Def. 7.1.2].

Property 2. If $\mathbf{A}$ and $\mathbf{B}$ are both psd, then so is $\mathbf{C}=\mathbf{A} \otimes \mathbf{B}$ [55, p.254 Fact 7.4.15].

Definition 5. An even function $f(t)$ is said to be positive definite if and only if for any $N$, for any $t_{1}, \ldots, t_{N}$ on $\mathbb{R}^{+}$, the matrix with entries $a_{\imath, \jmath}=f\left(\left|t_{\imath}-t_{\jmath}\right|\right)$ is psd. This can directly be derived from [56, p.58 Eq. 1.29] and Definition 2. 
Property 3 (Bochner's Theorem). A continuous even function $f(\tau)$ is positive definite if and only if there exists a non-decreasing bounded function $F(\omega), \omega \in \mathbb{R}^{+}$such that $[56$, p.92 Eq. 2.53]

$$
f(\tau)=\int_{0}^{\infty} \cos \omega \tau d F(\omega)
$$

Property 4. Every covariance matrix is necessarily $\operatorname{psd}^{1}$ and symmetric $[56$, p. 15 Eq. 0.36].

Property 5 (Pólya's Theorem). A bounded even function $f(\tau)$ with $f(\infty)=0$ that is concave (up) on $(0, \infty)$ is necessarily positive definite [56, p.136]. (The converse is not true.)

\subsubsection{Gaussian Random Variables and Processes}

We make extensive use of jointly Gaussian RVs and related concepts, which are welldescribed in [58]; it is thus important to understand these well.

Definition 6. A Gaussian vector is one that can be obtained by a linear transformation on a vector of independent Gaussian RVs.

This leads to two basic properties that can be used to construct Gaussian vectors:

Property 6. A vector of iid standard Gaussian RVs is Gaussian.

Property 7. A linear transformation on a Gaussian vector is a Gaussian vector.

From these two statements, the following property follows:

Property 8. Any Gaussian vector is fully specified by its mean vector and covariance matrix.

It is important to note that a vector of individually Gaussian RVs may not be a Gaussian vector. Therefore:

\footnotetext{
${ }^{1}$ Reference [57] says "positive definite", but this is too restrictive; whereas [56] also says "positive definite", but this is a difference in nomenclature, and clearly psd is meant.
} 
Definition 7. We call marginally Gaussian a vector of RVs that are individually Gaussian.

A Gaussian vector is thus a particular case of a marginally Gaussian vector.

Definition 8. If a vector $\left[X_{\imath}\right]_{l=1}^{N}$ is Gaussian, then we call $\left[e^{X_{2}}\right]_{\imath=1}^{N}$ a lognormal vector. Similarly, if a vector $\left[X_{\imath}\right]_{\imath=1}^{N}$ is marginally Gaussian, then we call $\left[e^{X_{2}}\right]_{\imath=1}^{N}$ a marginally lognormal vector.

Definition 9. A random process (RP) $X(t)$ is said to be Gaussian if, for any finitelength vector of real values $\left[t_{\imath}\right]_{l=1}^{N}$, the vector $\left[X\left(t_{\imath}\right)\right]_{l=1}^{N}$ is Gaussian. On the other hand, a RP is merely marginally Gaussian if that vector is merely marginally Gaussian.

Definition 10. If $X(t)$ is a Gaussian RP, then we call $e^{X(t)}$ a lognormal RP. If $X(t)$ is a marginally Gaussian RP, then we call $e^{X(t)}$ a marginally lognormal RP.

Definition 11. A RP $X(t)$ is said to be stationary if it is statistically invariant under a shift, i.e., the vectors $\left[X\left(t_{\imath}\right)\right]_{\imath=1}^{N}$ and $\left[X\left(t_{\imath}+\tau\right)\right]_{\imath=1}^{N}$ are statistically identical for any constant $\tau$.

Property 9. For any stationary RP $X(t)$, its auto-correlation function (if it exists) can be written $\mathbb{E}\{X(t) X(t+\tau)\}=R_{X}(\tau)$ for any $\tau$.

Together with Property 8, this leads to the following:

Property 10. A stationary Gaussian RP $X(t)$ is fully specified statistically by its mean $\mu$ and auto-correlation function $R_{X}(\tau)=\mathbb{E}\{X(t) X(t+\tau)\}$. A stationary lognormal RP $e^{X(t)}$ can be fully described analogously by the mean and auto-correlation function of $X(t)$.

We are also interested in two-dimensional RPs, usually called random fields, where stationarity is a somewhat more complex matter that needs to be defined more accurately as follows: 
Definition 12. A two-dimensional $\mathrm{RP} X(x, y)$ is said to be stationary [59] if it is statistically invariant under translation, i.e., the vectors $\left[X\left(x_{i}, y_{2}\right)\right]_{i=1}^{N}$ and $\left[X\left(x_{\imath}+u, y+v\right)\right]_{i=1}^{N}$ are statistically identical for any $u$ and $v$.

Property 11. For any stationary RP $X(x, y)$, its auto-correlation function can be written $\mathbb{E}\{X(x, y) X(x+u, y+v)\}=R_{X}(u, v)$ for any $u$ and $v$.

Together with Property 8 , this leads to the following:

Property 12. A stationary Gaussian $\mathrm{RP} X(x, y)$ is fully specified statistically by its mean $\mu$ and auto-correlation function $\mathbb{E}\{X(x, y) X(x+u, y+v)\}=R_{X}(u, v)$. A stationary lognormal RP $e^{X(x, y)}$ can be fully described analogously by the mean and auto-correlation function of $X(x, y)$.

Definition 13. A two-dimensional RP $X(x, y)$ is said to be separable [60] if its autocorrelation function may be written as $\mathbb{E}\{X(x, y) X(z, w)\}=R_{x, X}(x, z) R_{y, X}(y, w)$.

\subsubsection{Convergence of Random Variables}

Definition 14. A sequence $X_{1}, X_{2}, \ldots, X_{N}$ of RVs with cumulative distribution functions (cdfs) $F_{1}(x), F_{2}(x), \ldots, F_{N}(x)$ converges in distribution [61] to an $\mathrm{RV} X$ with cdf $F(x)$ if

$$
\lim _{N \rightarrow \infty} F_{N}(x)=F(x), \quad \forall x \in \mathbb{R} .
$$

We write: $X_{N} \stackrel{\mathcal{D}}{\longrightarrow} X$ as $N \rightarrow \infty$.

It is important to note that convergence in distribution means the convergence of the cdf at every point, but not necessarily of the probability density function (pdf), even at any one point, as can be seen in the addition of discrete RVs.

Theorem 1 (Slutsky's theorem [61]). Let $X_{N} \stackrel{\mathcal{D}}{\longrightarrow} X$ as $N \rightarrow \infty$ and $Y_{N} \stackrel{\mathcal{D}}{\longrightarrow} C$ a constant as $N \rightarrow \infty$. Then $X_{N} Y_{N} \stackrel{\mathcal{D}}{\longrightarrow} X C$ as $N \rightarrow \infty$. 
Two points are of note regarding Slutsky's theorem:

- The sequence $\left\{X_{N}\right\}$ need not be independent of the sequence $\left\{Y_{N}\right\}$.

- In general, this result does not hold if $\left\{Y_{N}\right\}$ also converges to a non-constant RV: the limit of the product of random sequences is not necessarily the product of the limits.

Theorem 2 (The Central Limit Theorem [61]). Let $X_{\imath}$ be a set of iid RVs with finite variance $\operatorname{VAR}\left\{X_{1}\right\}$

$$
\sum_{i=1}^{N} \frac{X_{\imath}-\mathbb{E}\left\{X_{1}\right\}}{\sqrt{N \mathbb{A R}\left\{X_{1}\right\}}} \stackrel{\mathcal{D}}{\longrightarrow} Z,
$$

where $Z$ is a Gaussian RV with mean 0 and variance 1 [61].

Theorem 3 (The (Weak) Law of Large Numbers [61]). Let $X_{\imath}$ be a set of iid RVs with $\operatorname{VAR}\left\{X_{1}\right\}<\infty$

$$
\sum_{i=1}^{N} \frac{X_{\imath}}{N} \stackrel{\mathcal{D}}{\longrightarrow} \mathbb{E}\left\{X_{1}\right\} .
$$

The CLT and the law of large numbers (LLN) are important tools in analysing the sum of very many RV, and therefore relevant to the analysis of the sum interference from many ISs. They are not, however, sufficient for our purpose, where we look at correlated shadowing, and consequently at correlated interference components. We therefore need to develop a more advanced limit theorem for our analysis, which we do in Section 4.1.1.

\subsubsection{Exchangeable Random Variables}

The theory of exchangeable RVs was introduced by De Finetti [62-64]. Exchangeable RVs are a central concept of this thesis, and can be seen as a generalisation of iid RVs. Intuitively, a set of exchangeable RVs is simply one where the index assignment is arbitrary, i.e., all permutations of the RVs have the same joint distribution. This 
leads to a very wide set of applications, given that for any system where there are elements of the same type (in our case, interferers) we see that the index assignment is indeed arbitrary.

Definition 15. A permutation $p(i), i=1, \ldots, N$ on a vector $\vec{x}=\left[x_{\imath}\right]_{i=1}^{N}$ is any bijective (one-to-one) mapping from the components of $\vec{x}$ onto itself.

Definition 16. A finite set of RVs $\left\{X_{\imath}\right\}_{\imath=1}^{N}$ is said to be exchangeable if the vectors $\left[X_{1}, X_{2}, \ldots, X_{N}\right]$ and $\left[X_{p(1)}, X_{p(2)}, \ldots, X_{p(N)}\right]$ have the same joint distribution for any permutation $p(i)$ on the vector $[1,2, \ldots, N]$.

Theorem 4 (Hewitt-Savage Generalisation of de Finetti's Theorem [65]). For any exchangeable set $\left\{X_{\imath}\right\}_{\imath=1}^{N}$, there exists a single RV $Z$ such that all $X_{\imath}$ 's are conditionally independent on $Z$. Equivalently, there exists a set of iid RVs $\left\{Y_{\imath}\right\}_{\imath=1}^{N}$ such that, for all $i, X_{\imath}$ can be written as a function of $Z$ and $Y_{\imath}$. 


\section{Chapter 2}

\section{Choosing a Good Shadowing Correlation Model}

Correlation in wireless shadowing is a significant step in obtaining more realistic channel propagation models. This chapter discusses in detail the existing literature on shadowing correlation and the existing correlation models, focusing on evaluating which models are mathematically consistent, while also making comments on their physical plausibility. The purpose of this chapter is to facilitate the choice and implementation of correlation models in works that involve shadowing.

Recent work on various aspects of wireless communications has indicated a wide gap between results obtained assuming independent shadowing paths and between those that introduce correlation in shadowing propagation models [45, 46, 66-68]. Older works show that shadowing correlation significantly affects handover behaviour [69-72], interference power [50,53,73-75] and consequently system performance [7678], and the performance of macrodiversity schemes [73,79-84]. Furthermore, shadowing in $\mathrm{dB}$ has been measured [43,79,85-99] to have significant correlation in various scenarios. Because of these two facts we believe that the general wireless community is becoming convinced of the importance of modeling correlation in shadowing $[18,41,45-47,67,68,75,84,100-106]$. This is part of a more general trend suggesting that most propagation models used in simulation (and analysis) work today are sometimes too simplistic and may lead to misleading results [107]. Shadowing 
correlation can also be positively exploited in some algorithms or protocols, e.g., for wireless positioning $[45,68,108]$, cognitive radio and spectrum sensing [106,109-111], or neighbour discovery [105] applications.

Meanwhile, there already exist several models for shadowing correlation. The questions of which model to use and how to simulate channel realisations accordingly are essential for every researcher wanting to implement correlation. However, because correlation models are based on estimating a complex phenomenon from a small data sample, they may lose some properties with respect to reality. In particular, some estimated models may not be mathematically feasible, and thus it would be impossible, in certain cases, to generate Monte Carlo samples from them.

In [103], it is deplored that this problem is rarely taken into account; yet it has received some attention in $[57,69,70,103,104,112,113]$. It has previously been addressed $[102,113,114]$ by slightly modifying particular correlation matrices so that they become feasible (specifically, psd for lognormal shadowing). However, we prefer to address the problem at its root: let us use only those correlation models that always produce psd correlation matrices, as suggested in $[57,69,103]$. This has several advantages:

1. A faster implementation time, as no provision for correcting non-psd matrices needs to be made.

2. Probably a faster execution time for every realisation, as no decisions or corrections need to be made during the Monte Carlo simulation.

3. Mathematical consistency and elegance: if a model is feasible, it is safe for mathematical analysis $[47,53,72-74,82,83,109,115-120]$.

If we begin with a set of independent RVs, and construct correlated shadowing variables by combining them in some way, we can always calculate their correlation 
structure. However, the inverse operation cannot always be performed: there does not always exist a construction to obtain a desired correlation structure. Those that cannot be constructed are termed not feasible.

\subsection{Types of Correlation}

In wireless communications, shadowing is a phenomenon that corresponds to a random variability of the power gain over a (directed) propagation path. A transmitting node radiates a radio signal toward a receiver node. The receiver may receive a desired signal, or an interfering one. Shadowing represents the variability of the logarithm of the received power around its expected value: we denote this quantity $S_{\imath}$, with $\operatorname{VAR}\left\{S_{\imath}\right\}<\infty$. The small-scale effect of fading has been removed in the spatial dimension by averaging over a local region [103] of about 50 wavelengths $[57,118]$, that is, approximately $8-34$ meters for classic cellular channels $[79,85-87,89,97,98]$.

Consider two directed paths, $\overrightarrow{X_{1} Y_{1}}$ and $\overrightarrow{X_{2} Y_{2}}$, with shadowing values $S_{1}$ and $S_{2}$ respectively. Their correlation coefficient is defined as

$$
\rho_{1,2}=\frac{\mathbb{E}\left\{S_{1} S_{2}\right\}}{\sqrt{\operatorname{VAR}\left\{S_{1}\right\} \operatorname{VAR}\left\{S_{2}\right\}}}
$$

In general, correlation may exist for any set of four points $X_{1} \neq Y_{1}, X_{2} \neq Y_{2}$. However, we usually examine correlation under more specific scenarios.

\subsubsection{Scenarios}

Shadowing correlation can occur in various particular scenarios, specifically in peerto-peer links $[40,44,45]$, in indoor, cross-floor, and indoor-outdoor links [87,90], and in satellite ground communications [91, 121-123]. It should be noted however that most of the literature on correlation in shadowing is driven by cellular (usually urban) 

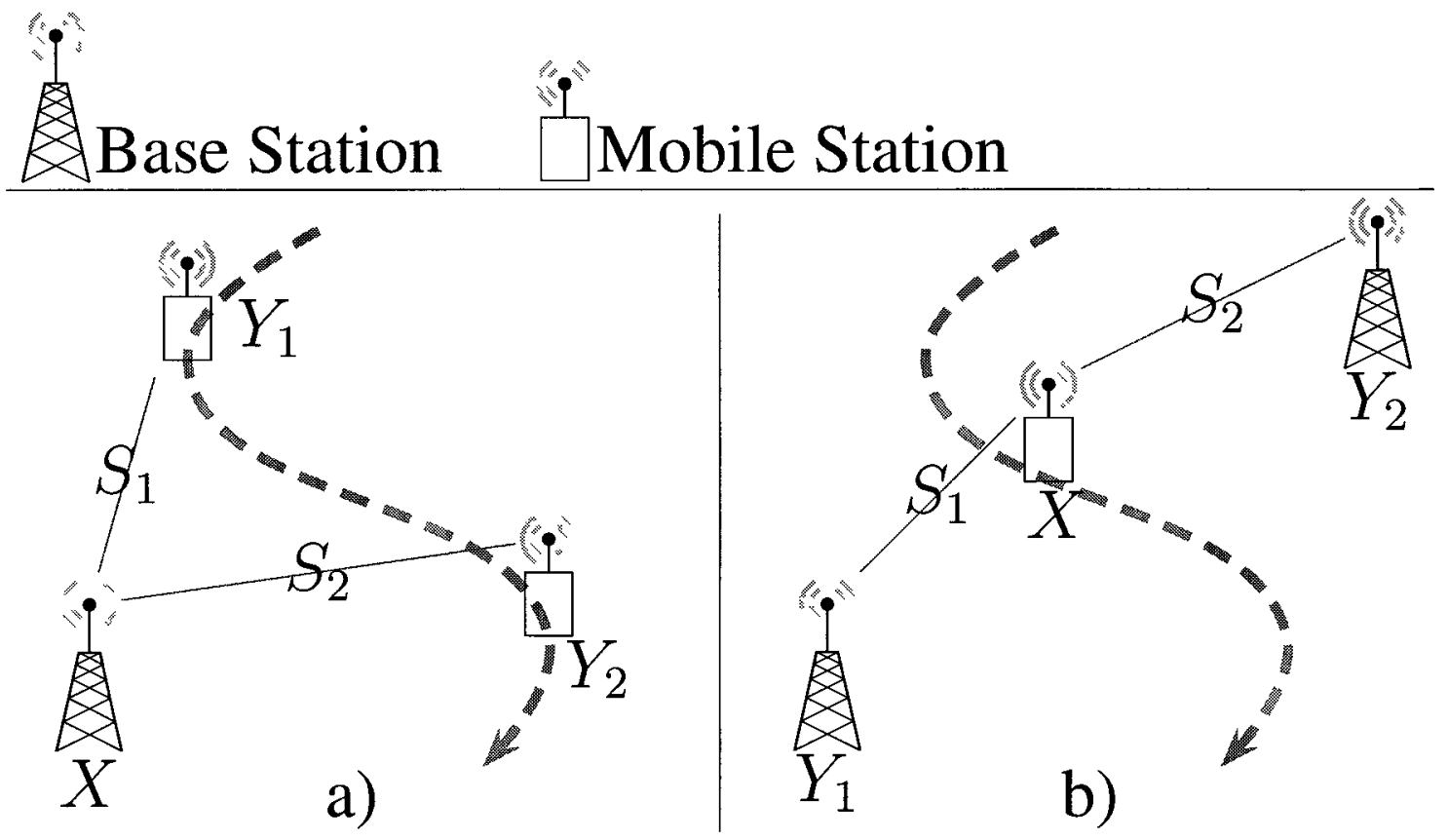

Figure 2.1: a) Shadowing auto-correlation for a mobile $Y(t)$, b) Shadowing crosscorrelation for a mobile $X$.

scenarios, where the base-station/mobile and uplink/downlink dualities apply. In cellular communications, the distinction between auto-correlation and cross correlation applies [103]. Cross-sector correlation has also been considered [48].

An important consideration for satellite and indoor scenarios is that propagation is usually considered in three, not just two, Cartesian dimensions. In this work we analyse two-dimensional channel models only, although the analysis usually translates easily to three dimensions.

\subsubsection{Auto-Correlation}

Also called serial correlation [124], this model considers a transmitting base-station $X$, received by the same moving mobile $Y$ at two moments in time, $t_{1}$ and $t_{2}$, and at distinct locations $Y_{1}=Y\left(t_{1}\right)$ and $Y_{2}=Y\left(t_{2}\right)$. Alternatively, the signal may be 
received by two distinct mobiles, $Y_{1}$ and $Y_{2}$, at the same moment in time. These scenarios may also be reversed to consider the uplink: either the same mobile transmits to the same base station $X$ at moments $t_{1}$ and $t_{2}$ at locations $Y_{1}$ and $Y_{2}$ respectively; or the same base station $X$ simultaneously receives from two distinct mobiles at locations $Y_{1}$ and $Y_{2}$. All these four cases can come under the category of auto-correlation and are illustrated in Figure 2.1 a).

\subsubsection{Cross-Correlation}

This is the central object of our study. Cross-correlation, or site-to-site correlation [124], considers two transmitting base stations, $Y_{1}$ and $Y_{2}$, that transmit to a common mobile receiver $X$. Alternatively, cross-correlation can consider a mobile transmitter $X$ whose signal is picked up by two base stations, $Y_{1}$ and $Y_{2}$. These are illustrated in Figure $2.1 \mathrm{~b})$.

Now, observing the previous section on auto-correlation and the systematic nomenclature we gave to nodes ( $X$ a common node, and $Y_{1}$ and $Y_{2}$ nodes on two separate links), we may abstract from the nature of these nodes and the link direction, and conclude that auto-correlation and cross-correlation are very similar in the mathematical sense and can be studied in the same manner. Furthermore, we see in $[104,125]$ how an auto-correlation model can be used to model cross-correlation also. We therefore assume that $X$ is the common node to all paths, which we locate at the origin for simplicity, and all links are between $X$ and the points $Y_{i}$.

\subsubsection{Mathematical Synthesis of Auto- and Cross-Correlation}

Auto- and cross-correlation models can be generically described as follows. Consider $N$ points $Y_{1}, \ldots, Y_{N}$ located on a plane at positions $\vec{r}_{1}, \ldots \vec{r}_{N}$ with $\vec{r}_{i} \in \mathbb{R}^{2} \backslash\{0\}$. We assume, without loss of generality, that the common point $X$ is located at the

origin, and thus $\vec{r}_{i}=\overrightarrow{X Y}_{i}$. Consider $S_{i}$ the logarithm of the power attenuation due 


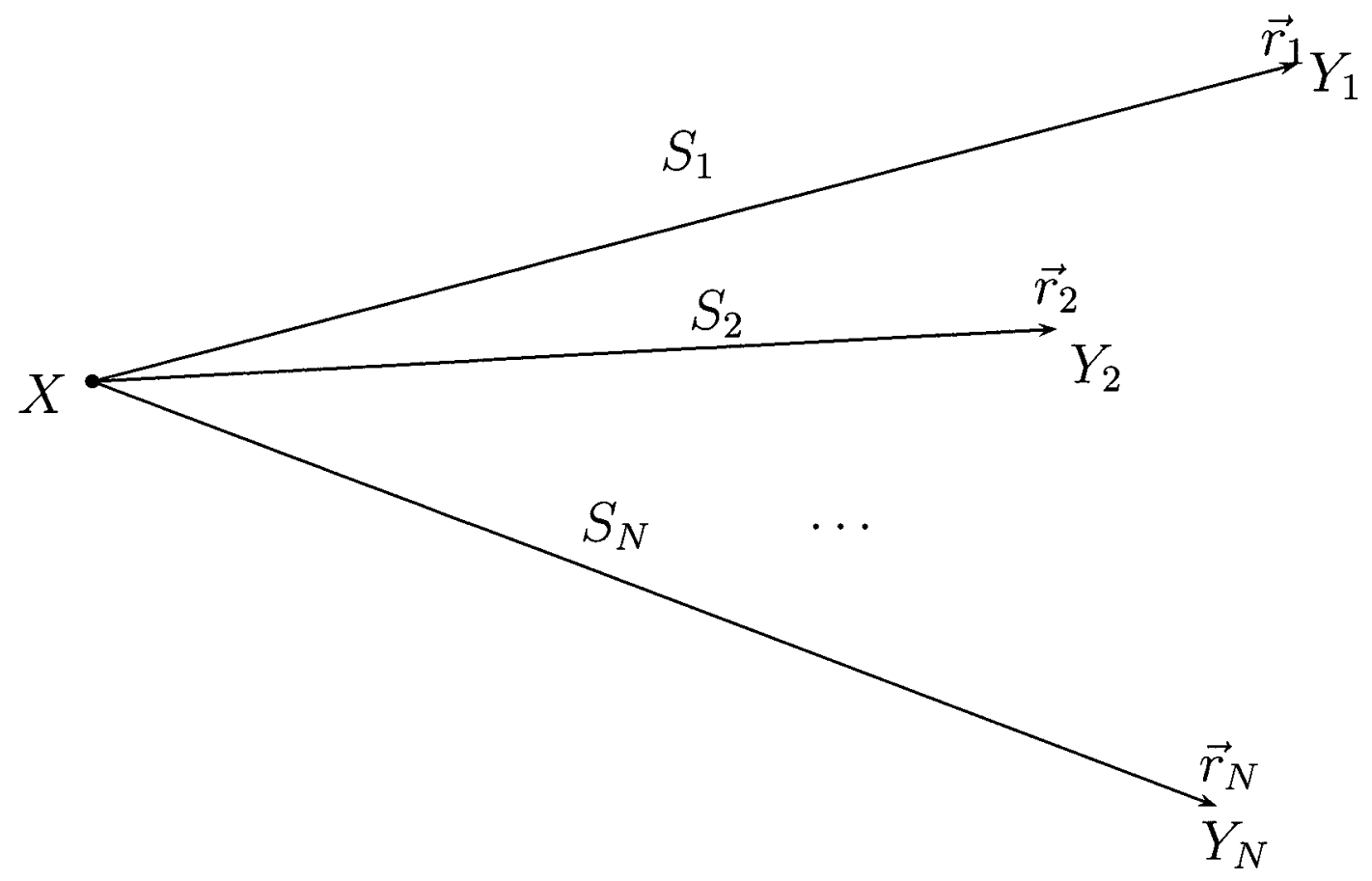

Figure 2.2: Generic geometry of shadowing auto-and cross-correlation.

to shadowing on each path $\vec{r}_{\imath}$ (see Figure 2.2). ${ }^{1}$

We observe that both auto- and cross-correlation can be viewed under this same framework, particularly given the common notation that we introduced for Figure 2.1. It is this common model that we intend to study. Then the shadowing model can be entirely specified by the pair of functions $\left(\sigma_{\mathrm{s}}^{2}(\vec{r}), h\left(\vec{r}_{1}, \vec{r}_{2}\right)\right)$.

\subsubsection{Generalised Correlation}

If we allow total freedom for the positions of the two paths, then the correlation model becomes more complex; in fact, it becomes a function of up to eight free variables (four free positions on a two dimensional plane).

We do not study this type of correlation for two reasons: first, because of its increased complexity; and second, because the methods given in literature begin with

\footnotetext{
${ }^{1}$ At this moment, we need not commit to any particular shadowing distribution. We require only the condition that $\operatorname{VAR}\left\{S_{\imath}\right\}<\infty$.
} 
an explicit construction of the shadowing realisations, and hence it is not necessary to study their feasibility: they are always feasible. Contrast this with the correlation models studied here. In Section 2.6.1, it is necessary to factorise a matrix, which is not always possible, and thus the method is implicit and feasibility must be studied.

Such methods rely of generating shadowing maps or fields in some way. Three algorithms for doing so are the Sum-of-Sinusoids (SoS) algorithm [40], the Network Shadowing (NeSh) method [44-47], and the over-obstacle multiple-edge diffraction model [39].

\subsubsection{Time-Correlation}

Time-correlation is different in that it only considers one path $\overrightarrow{X Y}$, but looks at the correlation between the shadowing at various moments in time. The shadowing can thus be represented as a $\operatorname{RP} S(t)[71,120]$. The feasibility of the model is a simple problem here, because the problem simply evolves in one dimension: time. The correlation model is then specified by the auto-correlation function of $S(t)$, and the model is psd if and only if the auto-correlation function is psd. We are not aware of any measurements of correlation in time only, and it is not evident if shadowing changes significantly over a fixed path.

It should be understood that shadowing evolution in time should (like in space) have fast fading removed through time-averaging of some duration.

\subsubsection{Uplink-Downlink Correlation}

Consider the path $\overrightarrow{X Y}$, and then its return path $\overrightarrow{Y X}$. By channel symmetry, one might conclude that the shadowing experienced in both directions is identical, which would correspond to a correlation coefficient of $\rho_{\mathrm{ud}}=1$. In practice, measurements indicate a high degree of correlation: $\rho_{\mathrm{ud}} \geq 0.66$ [95]. From these measurements, [126] assumed $\rho_{\mathrm{ud}}=0.7$. Furthermore, [107] demonstrated asymmetry but positive 
correlation in link connectivity, which can be interpreted as unequal but correlated shadowing in each direction.

\subsection{Positive Semidefiniteness of Correlation Models}

From Property 4, for $\mathbf{K}$ to be a valid covariance matrix of $\vec{S}$, it is a necessary (but not always sufficient, as seen in Section 2.6 ) that $\mathbf{K}$ be psd ( $\mathbf{K}$ is already symmetric by construction).

Definition 17. We say that a shadowing model $\left(\sigma_{\mathrm{s}}^{2}(\vec{r}), h\left(\vec{r}_{1}, \vec{r}_{2}\right)\right)$ is psd if $\forall N, \forall\left[\vec{r}_{1}, \ldots, \vec{r}_{N}\right] \in$ $\left(\mathbb{R}^{2} \backslash\{0\}\right)^{N}$, the correlation matrix $\mathbf{K}$ is always psd.

\subsubsection{Models with Variable Shadowing Variance}

Theorem 5. If a model $\left(1, h\left(\vec{r}_{1}, \vec{r}_{2}\right)\right)$ with constant $\log$-variance 1 is psd, then for any $\sigma_{\mathrm{s}}^{2}(\vec{r})$, the model $\left(\sigma_{\mathrm{s}}^{2}(\vec{r}), h\left(\vec{r}_{1}, \vec{r}_{2}\right)\right)$ is also psd. Conversely, if the model $\left(\sigma_{\mathrm{s}}^{2}(\vec{r}), h\left(\vec{r}_{1}, \vec{r}_{2}\right)\right)$ is psd and $\sigma_{\mathrm{s}}^{2}(\vec{r})>0 \forall \vec{r} \in \mathbb{R}^{2} \backslash\{0\}$, then the model $\left(1, h\left(\vec{r}_{1}, \vec{r}_{2}\right)\right)$ with constant $\log$-variance 1 is psd.

Proof. Consider $N$ shadowing paths $\vec{r}_{\imath} \in \mathbb{R}^{2} \backslash\{0\}$ and a shadowing model $\left(\sigma_{\mathrm{s}}^{2}(\vec{r}), h\left(\vec{r}_{1}, \vec{r}_{2}\right)\right)$ where $\left(1, h\left(\vec{r}_{1}, \vec{r}_{2}\right)\right)$ is a psd shadowing model. We call $\mathbf{H}$ the $N \times N$ matrix with entries $h\left(\vec{r}_{\imath}, \vec{r}_{j}\right)$, and $\vec{s}$ the column vector with entries $\sigma_{\mathrm{s}}\left(\vec{r}_{\imath}\right)$. Then the correlation matrix of $\vec{S}$ can be written

$$
\mathbf{K}=\left(\vec{s} \vec{s}^{\mathrm{T}}\right) \circ \mathbf{H}
$$

Now the matrix $\vec{s}^{\mathrm{T}}$ is psd as can be seen from Definition 2: $\vec{x}^{\mathrm{T}}\left(\vec{s}^{\mathrm{T}}\right) \vec{x}=\left(\vec{x}^{\mathrm{T}} \vec{s}\right)^{2} \geq$ $0 \forall \vec{x} \in \mathbb{R}^{N}$. Also, since $\left(1, h\left(\vec{r}_{1}, \vec{r}_{2}\right)\right)$ is psd, it follows that $\mathbf{H}$ is psd. Applying the Schur product theorem, we find that $\mathbf{K}$ is always psd, which implies that the model $\left(\sigma_{\mathrm{s}}^{2}(\vec{r}), h\left(\vec{r}_{1}, \vec{r}_{2}\right)\right)$ is psd. 
To prove the converse, we write

$$
\mathbf{H}=\left(\vec{z} \vec{z}^{T}\right) \circ \mathbf{K}
$$

where $\vec{z}$ is the column vector with entries $\sigma_{\mathrm{s}}^{-1}\left(\vec{r}_{i}\right)$, and the proof is analogous. Of course, here the additional requirement that $\sigma_{\mathrm{s}}(\vec{r}) \neq 0, \forall \vec{r} \in \mathbb{R}^{2} \backslash\{0\}$ is required.

Thus in order to study the positive semidefiniteness of a shadowing model, it is sufficient to study the correlation function $h\left(\vec{r}_{1}, \vec{r}_{2}\right)$ in isolation, which simplifies the problem. We therefore say that $h$ is psd if $(1, h)$ is psd.

\subsubsection{Methods for Proving Positive Semidefiniteness}

While we do not have a general criterion for proving that some given $h$ is psd or not, these few approaches nevertheless help us analyse most particular cases:

1. If there exists an explicit constructive algorithm for generating data according to $h$, then $h$ is necessarily psd, since the resulting covariance matrix is always psd (see Property 4).

2. If there exists at least one choice of $\left[\vec{r}_{1}, \ldots, \vec{r}_{N}\right]$ for which $\mathbf{K}$ is not psd, then $h$ is not psd. For this test, we need $N \geq 3$, since every correlation matrix of size 2 is psd:

$$
\left[\begin{array}{ll}
1 & \rho \\
\rho & 1
\end{array}\right]=\left[\begin{array}{ll}
u & v \\
v & u
\end{array}\right]^{2}, u=\frac{1}{2} \sqrt{1+\rho}+\frac{1}{2} \sqrt{1-\rho}, v=\frac{1}{2} \sqrt{1+\rho}-\frac{1}{2} \sqrt{1-\rho}
$$

However, $N=3$ may not be enough [113].

3. Several theorems can also be used to prove that some $h$ is psd, as seen in the next section. 


\subsubsection{One-Parameter and Separable Correlation}

Proving that isotropic models (functions of $d=\left\|\vec{r}_{1}-\vec{r}_{2}\right\|$ only, with $\vec{r}_{\imath} \in \mathbb{R}^{2}$ ) are psd is not trivial. Indeed, while for all psd models $h(d)$, the function $h(x)$ on $\mathbb{R}^{+}$is positive definite, the converse is not true, and tighter conditions are needed [56, p.361]. The following two Theorems are examples of tests that can be applied to verify that $h(d)$ is psd:

Theorem 6. A one-parameter correlation model $h(d)$ with $d=\left\|\vec{r}_{1}-\vec{r}_{2}\right\|$ with $\vec{r}_{\imath} \in$ $\mathbb{R}^{2}$ is psd if ${ }^{2}$ the integral

$$
f(\omega)=\int_{0}^{\infty} h(x) J_{0}(\omega x) x d x
$$

exists and is non-negative $\forall \omega \geq 0 . J_{0}(x)$ is here the Bessel function of the first kind of order $0 .[56$, p.357]

Theorem 7. A one-parameter correlation model $h(d)$ with $d=\left\|\vec{r}_{1}-\vec{r}_{2}\right\|$ with $\vec{r}_{\imath} \in$ $\mathbb{R}^{2}$ is psd if ${ }^{2}$

1. the function $h(x)$ with $x \in \mathbb{R}^{+}$is positive definite and

2. the Fourier transform $f(\omega)$ of $h(|x|)$ is non-increasing on $\omega \in(0, \infty)$.

The converse is not true. [56, p.361]

Many other useful properties of isotropic correlation models are found in [56, Chapter 22].

Theorem 8. A one-parameter correlation model $h(\theta)$ with $\theta=\left|\angle \vec{r}_{1}-\angle \vec{r}_{2}+2 k \pi\right|, k \in$ $\mathbb{Z}$ such that $\theta \in\left[0^{\circ}, 180^{\circ}\right]$ is psd if it may be written as

$$
h(\theta)=\sum_{n=0}^{\infty} a_{n} \cos (n \theta),
$$

\footnotetext{
${ }^{2}$ These two theorems apply for shadowing on $\mathbb{R}^{2}$, and take a more restricted form on $\mathbb{R}^{3}[56]$.
} 
for some non-negative and bounded sequence $a_{0}, a_{1}, \ldots$ with $0<\sum_{n=0}^{\infty} a_{n}<\infty$.

Proofs are given in $[57,112]$.

Theorem 9. A one-parameter correlation model $h(|x|)$ with $|x|=\left|g\left(\vec{r}_{1}\right)-g\left(\vec{r}_{2}\right)\right|$ for some function $g: \mathbb{R}^{2} \backslash\{0\} \mapsto \mathbb{R}$ is psd if it may be written as

$$
h(x)=\int_{0}^{\infty} \cos (2 \pi x \omega) f(\omega) d \omega
$$

for some non-negative and finite-area $f(\omega)$ on $0<\omega<\infty$.

Proof. From Bochner's Theorem, $h(x)$ in (2.7) is a psd function. From Definition 5, it follows that for any $N$ the matrix $\mathbf{H}_{N \times N}$ with entries $\rho_{\imath, \jmath}=h\left(\left|t_{\imath}-t_{\jmath}\right|\right), t_{\imath}=g\left(\vec{r}_{\imath}\right)$ is psd.

Theorem 10. If a correlation model $h$ may be written as

$$
h\left(\vec{r}_{1}, \vec{r}_{2}\right)=h_{1}\left(\vec{r}_{1}, \vec{r}_{2}\right) h_{2}\left(\vec{r}_{1}, \vec{r}_{2}\right)
$$

with $h_{1}$ and $h_{2}$ both psd, then $h$ is also psd.

Proof. Let $\mathbf{H}, \mathbf{H}_{\mathbf{1}}$, and $\mathbf{H}_{\mathbf{2}}$ be the $N \times N$ matrices with entries $h\left(\vec{r}_{\imath}, \vec{r}_{\jmath}\right), h_{1}\left(\vec{r}_{\imath}, \vec{r}_{\jmath}\right)$, and $h_{2}\left(\vec{r}_{\imath}, \vec{r}_{\jmath}\right)$ respectively. We may then write $\mathbf{H}=\mathbf{H}_{1} \circ \mathbf{H}_{2}$. Now since $h_{1}$ and $h_{2}$ are psd, it follows that $\mathbf{H}_{\mathbf{1}}$ and $\mathbf{H}_{\mathbf{2}}$ are both psd. Applying the Schur product theorem, we have that $\mathbf{H}$ is also psd, which implies that $h$ is psd.

A similar argument was given in [57], but for positive definite instead of psd matrices.

\subsubsection{Incorporating Time and Uplink-Downlink Correlation}

As seen in Section 2.1.6, shadowing may evolve in time in a correlated manner. Also, as seen in Section 2.1.7, shadowing may be different on the same propagation path in 
both directions, though these are usually highly correlated. We show that, given a positive definite temporal auto-correlation $R_{S}(\tau)$ and a psd spatial correlation model $h$, and assuming correlation separability between the spatial, time, and uplink-downlink dimensions, the resulting combined correlation matrix is always psd. Combining cross-correlation with temporal auto-correlation was described in [71].

Consider a common node $X$ and $N$ nodes $Y_{1}, \ldots, Y_{N}$. Let $S_{\imath}(t)$ be the shadowing on path $\overrightarrow{X Y}_{\imath}$ at time instant $t$ with variance $\sigma_{\imath}^{2}=\sigma_{\mathrm{s}}^{2}\left(\vec{r}_{\imath}\right)$, and $\tilde{S}_{\imath}(t)$ the shadowing on the return path $\overrightarrow{Y_{\imath} X}$, with variance $\tilde{\sigma}_{\imath}^{2}=\tilde{\sigma}_{\mathrm{s}}^{2}\left(\vec{r}_{\imath}\right)$.

Consider the spatial correlation function $h$ such that $\rho_{\imath, \jmath}=h\left(\vec{r}_{\imath}, \vec{r}_{\jmath}\right), \imath \neq \jmath$ and $\rho_{\imath, \imath}=1:$

$$
\begin{aligned}
\mathbb{E}\left\{S_{\imath}(t) S_{\jmath}(t)\right\} & =\sigma_{\imath} \sigma_{\jmath} \rho_{\imath, \jmath} \quad \forall t \\
\mathbb{E}\left\{\tilde{S}_{\imath}(t) \tilde{S}_{\jmath}(t)\right\} & =\tilde{\sigma}_{\imath} \tilde{\sigma}_{\jmath} \rho_{\imath, \jmath} \quad \forall t .
\end{aligned}
$$

We assume that the correlation between the two directions of the same path is constant, as in [126]:

$$
\mathbb{E}\left\{S_{\imath}(t) \tilde{S}_{\imath}(t)\right\}=\sigma_{\imath} \tilde{\sigma}_{\imath} \rho_{\mathrm{ud}} \quad \forall \imath \forall t
$$

Consider also the normalised temporal auto-correlation function $\zeta(\tau)$ :

$$
\frac{\mathbb{E}\left\{S_{\imath}(t) S_{\imath}(t+\tau)\right\}}{\sigma_{\imath}^{2}}=\frac{\mathbb{E}\left\{\tilde{S}_{\imath}(t) \tilde{S}_{\imath}(t+\tau)\right\}}{\tilde{\sigma}_{\imath}^{2}}=\zeta(\tau) \quad \forall \imath \forall t
$$

where $\zeta(\tau)$ is positive definite and $\zeta(0)=1$.

We further assume separability of cross-correlation and uplink-downlink correlation, so that the correlation terms can be found as follows:

$$
\begin{aligned}
& \frac{\mathbb{E}\left\{S_{\imath}\left(t_{1}\right) S_{\jmath}\left(t_{2}\right)\right\}}{\sigma_{\imath} \sigma_{\jmath}}=\frac{\mathbb{E}\left\{\tilde{S}_{\imath}\left(t_{1}\right) \tilde{S}_{\jmath}\left(t_{2}\right)\right\}}{\tilde{\sigma}_{\imath} \tilde{\sigma}_{\jmath}}=\rho_{\imath, \jmath} \zeta\left(t_{2}-t_{1}\right), \\
& \mathbb{E}\left\{S_{\imath}\left(t_{1}\right) \tilde{S}_{\jmath}\left(t_{2}\right)\right\}=\sigma_{\imath} \tilde{\sigma}_{\jmath} \rho_{\mathrm{ud}} \rho_{\imath, \jmath} \zeta\left(t_{2}-t_{1}\right) .
\end{aligned}
$$


From this equation we see that separability implies that the uplink and downlink cross-correlation models must be the same: $h=\tilde{h}$.

Consider $M$ time instances $t_{1}, \ldots, t_{M}$. Then the correlation matrix of $S_{1}\left(t_{1}\right)$, $\tilde{S}_{1}\left(t_{1}\right), \ldots, S_{N}\left(t_{1}\right), \tilde{S}_{N}\left(t_{1}\right), \ldots, S_{1}\left(t_{M}\right), \tilde{S}_{1}\left(t_{M}\right), \ldots, S_{N}\left(t_{M}\right), \tilde{S}_{N}\left(t_{M}\right)$ is

$$
\overline{\mathbf{K}}_{2 N M \times 2 N M}=\left[\zeta\left(t_{\jmath}-t_{\imath}\right)\right]_{M \times M} \otimes\left(\left(\overrightarrow{\breve{s} \breve{s}^{T}}\right) \circ\left(\left[\rho_{\imath, \jmath}\right]_{N \times N} \otimes\left[\begin{array}{cc}
1 & \rho_{\mathrm{ud}} \\
\rho_{\mathrm{ud}} & 1
\end{array}\right]\right)\right),
$$

where $\vec{s}$ is the column vector with entries $\sigma_{1}, \tilde{\sigma}_{1}, \ldots, \sigma_{N}, \tilde{\sigma}_{N}$.

Theorem 11. Given a psd correlation function $h$, uplink and downlink shadowing variances $\sigma_{\mathrm{s}}^{2}(\vec{r}), \tilde{\sigma}_{\mathrm{s}}^{2}(\vec{r})$, an uplink-downlink correlation coefficient $-1 \leq \rho_{\mathrm{ud}} \leq 1$, and a normalised shadowing positive definite time auto-correlation function $\zeta(\tau)$ with $\zeta(0)=1$, and assuming the correlation is separable as in (2.12), we can conclude that the composite correlation model is psd.

Proof. Consider (2.13). The matrix $\overleftrightarrow{\breve{s}^{\mathrm{s}}} \breve{\mathrm{S}}^{\mathrm{T}}$ is always psd, as seen in the proof of Theorem 5. The matrix $\left[\zeta\left(t_{1}-t_{2}\right)\right]_{M \times M}$ is psd because $\zeta(\tau)$ is positive definite, and the matrix $\left[\rho_{\imath, j}\right]_{N \times N}$ is psd because $h$ is psd. Finally, all $2 \times 2$ correlation matrices are psd, as seen in (2.4). Both the Hadamard and the Kronecker products are psd if both their factors are psd as seen in Properties 1 and 2. It follows that $\overline{\mathbf{K}}$ is psd.

Simply speaking, one can incorporate (separable) directional and time correlation without upsetting the positive semidefiniteness of the correlation model. Other dimensions, notably frequency [106], may also be similarly incorporated.

One can note that the Kronecker product has been similarly used in [127] in a multi-antenna context, and in [103] for correlation between shadowing, delay spread, and angle spread, all under the assumptions of separability, which is natural when no further information is available [103]. 


\subsection{Estimating the Correlation Function from Measured Data}

In order to better understand why estimated models may be psd or not, it is worthwhile to examine some aspects of how these models are constructed from measured data.

\subsubsection{Auto-Correlation as Mixed Time-Space Measurements}

Auto-correlation is measured [43,85,86,88,92,96-99] by taking a single moving mobile $Y$ with velocity $v$ in uniform rectilinear motion, measuring the power loss between $Y$ and a base station $X$, correcting for pathloss and small-scale fading, and considering the shadowing $S$ along the path $\overrightarrow{X Y}$ (or $\overrightarrow{Y X}$ ) as a process of time $S(t)$ (equivalently, of space: $S\left(d_{\mathrm{m}}\right)$, with $\left.d_{\mathrm{m}}=v t[128,129]\right)$. The underlying assumption is that shadowing over a fixed link does not vary with time (or perhaps varies little or very slowly compared to variation when in motion). More specifically, consider the shadowing $S(\vec{r}, t)$ as would be experienced by a virtual mobile $\tilde{Y}$ at time $t$ and location $\vec{r}$. Now let the real mobile $Y$ begin at location $\vec{r}_{0}$ at time $t=0$ and move in uniform rectilinear motion with velocity $\vec{v}$. Thus its location at time $t$ is $\vec{r}=\vec{r}_{0}+t \vec{v}$. The observed shadowing process at $Y$ is thus $S(t)=S\left(\vec{r}_{0}+t \vec{v}, t\right)$ or, equivalently, $S\left(d_{\mathrm{m}}\right)=$ $S\left(\vec{r}_{0}+d_{\mathrm{m}} \vec{v} / v, d_{\mathrm{m}} / v\right)$. Clearly, the observed process varies in both time and space, and assuming $S(\vec{r}, t)$ varies in $t$ when $\vec{r}$ is fixed (see Section 2.1.6), these measurements do not directly translate into a correlation model. For example, a different model $S\left(d_{\mathrm{m}}\right)$ might be extracted if the velocity $v$ is changed. This is generally true for other motion trajectories.

An additional issue about auto-correlation models is the level-crossing rate, which is only properly defined for some models. This topic is addressed in [117,118,129-131], and we do not explore it here further. 


\subsubsection{Cross-Correlation as Spatial-Only Correlation}

Several field measurements have been performed $[79,85,88,89,93,94,96-98]$ in order to gather data about the true physical correlation model, which we call $\bar{h}$. Field measurements produce a set of data points, from which an estimate of the crosscorrelation function, which we call $\hat{h}$, is obtained by fitting the data with plausiblelooking simple mathematical functions. It must be understood that $\bar{h}$ is always a psd model, by its very mathematical definition. It was argued in [113] that a particular conveniently psd model $\hat{h}$ may not reflect the true correlation accurately. This is true. However, because $\bar{h}$ must be psd, it is likely that there exists some psd model $\hat{h}$ that is close to $\bar{h}$. Therefore, we argue that it is always best and possible to choose a psd model $\hat{h}$ that is also close to $\bar{h}$.

In general, $\bar{h}\left(\vec{r}_{1}, \vec{r}_{2}\right)$ is a function of four variables. However, estimating a fourvariable function accurately requires many more points than can realistically be obtained from costly and cumbersome field measurements. Additionally, every point of the function $\bar{h}$ is itself estimated as an expectation, and requires several data points. Two consequences follow:

1. The measurement data is usually grouped by collapsing them from four variables onto a single one.

2. On that one variable, the curve-fitting is still relatively crude $[43,88,91,92,94$, 99].

These two approximation steps further distance $\hat{h}$ from the $\bar{h}$ and may cause the model $\hat{h}$ to be non-psd. Nevertheless, for practical reasons, it is simplest to measure correlation along one dimension only [56, pp. 358-359]. 


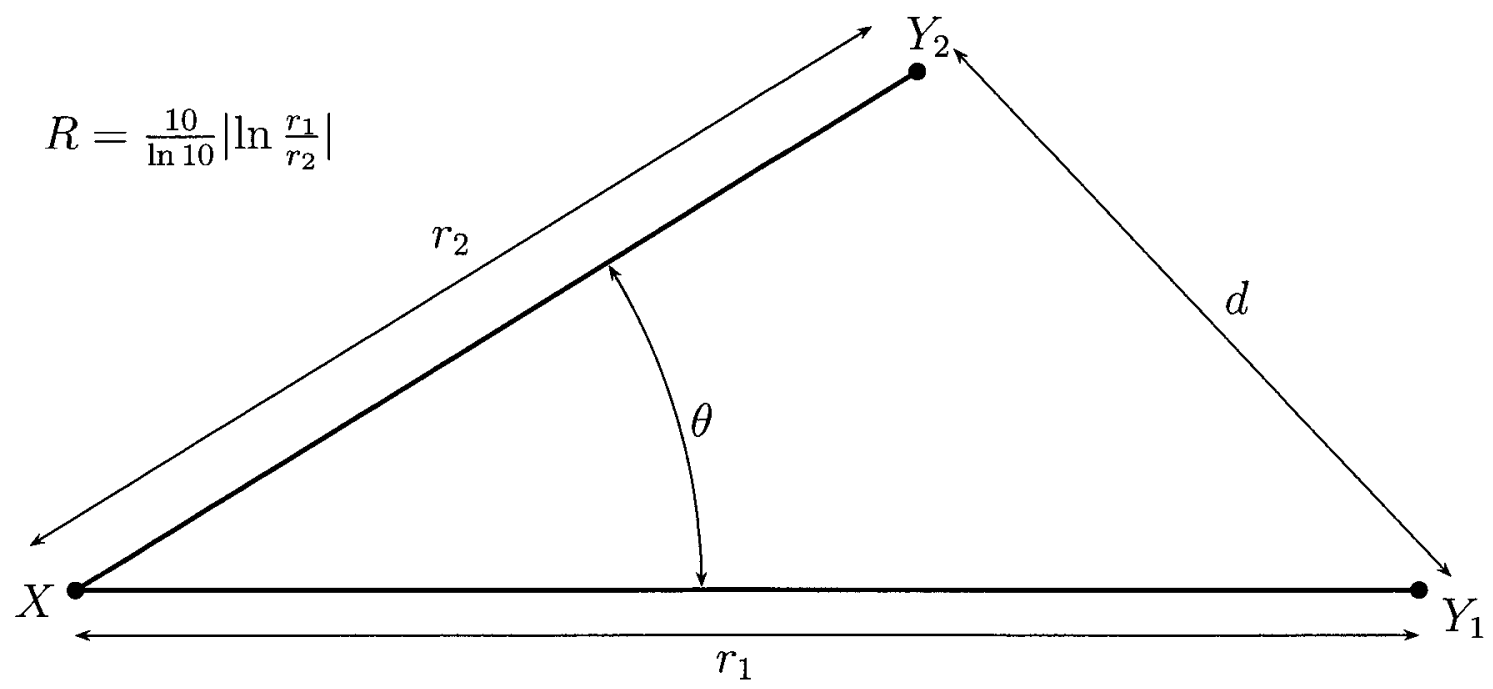

Figure 2.3: A pair of auto- or cross-correlated paths, with the most relevant dimensional variables: $d, \theta, R$.

\subsubsection{Collapsing Correlation onto One Dimension}

The measurements can be grouped along one variable in several ways. In such cases, $\hat{h}$ is expressed as a function of a single free variable. The most common forms are:

1. Absolute distance (between $Y_{1}$ and $Y_{2}$ ) [43,85,86,88,91,92,96-99]: $d=\left\|\vec{r}_{1}-\vec{r}_{2}\right\|$.

2. Angle (not oriented) of arrival separation $[57,85,88,93,94,96-98]: \theta=\left|\angle \vec{r}_{1}-\angle \vec{r}_{2}\right| \in$ $\left[0^{\circ}, 180^{\circ}\right]$.

3. Arrival distance ratio (in dB) $[48,57,93,97]: R=\left|10 \log _{10} r_{1} / r_{2}\right|=\frac{10}{\ln 10}\left|\ln r_{1}-\ln r_{2}\right|$.

These quantities are illustrated in Figure 2.3.

For correlation functions expressed in $d$, one may also estimate and fit the power spectral density (which is the Fourier transform of the auto-correlation function in differences of $d$ ) of the measured data to that of a particular model $[88,92]$. 


\subsection{Specific Correlation Models and Their Positive Semidef- initeness}

We have made a wide (if not exhaustive) investigation of correlation models $h$ used in literature. All existing models imply jointly lognormal shadowing (which is by no means a requirement of our analysis). Most models assume a constant shadowing variance $\sigma_{\mathrm{s}}^{2}(\vec{r})=\sigma_{\mathrm{s}}^{2}$, but not all $[66,99]$. As we have shown in Theorem 5 , the positive semidefiniteness of a shadowing model is separate from the shadowing spread function. As such, we may safely study the existing correlation models, while looking forward to more complex point-to-point shadowing spread models in the future.

The various existing models and their properties are summarised in Table 2.1. In particular, we show which models are psd. While [103] stated that "most" models are non-psd, we find that actually a slight majority of models are in fact psd.

The models are sorted according to the physical dimensions they take as parameters. A family tree of these models is illustrated in Figure 2.4. It should also be understood that these models are not necessarily mutually exclusive when they are expressed in different $(d, R, \theta)$ domains, since this implies a different reduction (inte-

gration) of the original four-dimensional model $\bar{h}$ onto one or two dimensions, possibly representing different projections of the same $\bar{h}$ onto those dimensions.

\subsubsection{Constant Model}

The simplistic model that assigns $\rho_{\imath, \jmath}=\rho, i \neq j$ :

$$
h\left(\vec{r}_{1}, \vec{r}_{2}\right)=\rho,
$$

with $0<\rho<1$, is sometimes used $[18,41,52,68,72-74,81,82,89,103,110,117,119$, $126,132](\rho=0.5[70,84,115,116])$ when more information is lacking. However, 


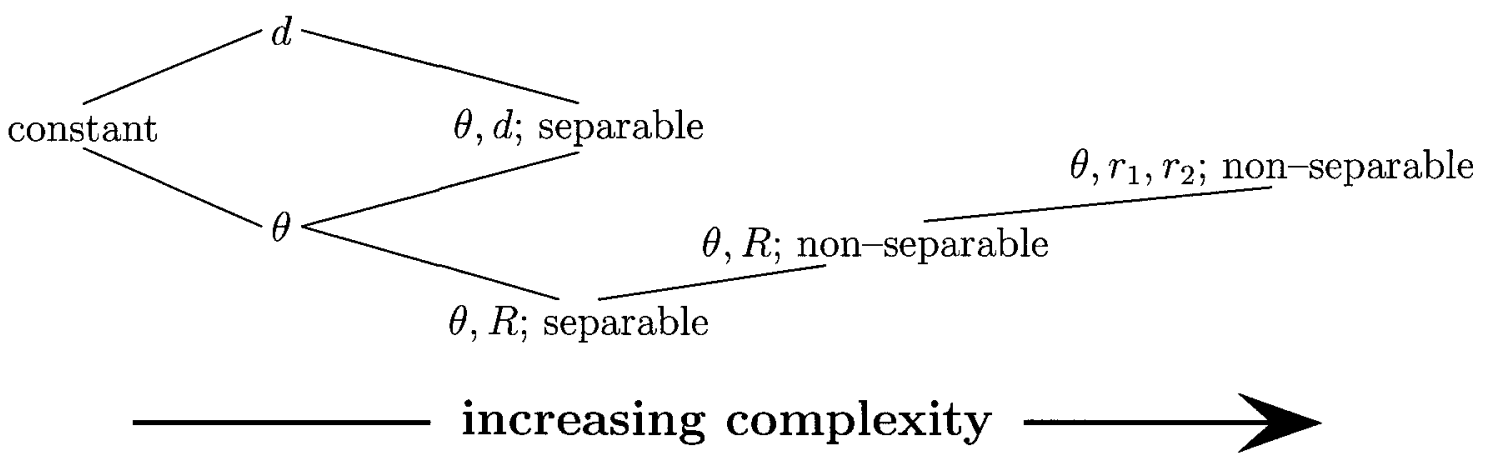

Figure 2.4: Shadowing correlation model families according to physical dimensions described in Figure 2.3. Other parameter combinations are possible but have not been encountered in literature.

[70] argued that this may be a too simplistic model, comparing simulations that use constant versus non-constant models. On the other hand, we have shown [53] that for a high number of highly-correlated lognormal-shadowed interferers, the total interference power may be well approximated with the knowledge of the average correlation coefficient only. (It is important to actually estimate this average. This was not done in [70].)

The model is claimed psd for $\rho=0.5$ in [103]. It does, in fact, give a psd $\mathbf{H}$ for $\rho \geq-1 /(N-1)$ [52], and so the model is psd according to Definition 17 for $0<\rho<1$ (and also $\rho=0,1$ ), but would be non-psd for $-1 \leq \rho<0$. It can be simulated quickly $[41,52]$ in the case of jointly lognormal correlated shadowing.

\subsubsection{Absolute Distance-Only Models}

All the auto-correlation models are expressed as a function of $d$ (equivalently, of time $t$, given a constant velocity $v$ ). 


\subsubsection{Exponential Model}

The most common auto-correlation model is a decaying exponential of distance $[10$, $41,43,57,69,71,86,91,96,98-100,103,105,106,108,109,118,122,124,125,128,130$, 133-136]. This model is often attributed to Gudmundson in 1991 [43], though the less-cited work by Marsan, Hess, and Gilbert in 1990 [86] also proposes this model, and measurements by Graziano in 1978 [85, Figs. 5 and 7] suggest it. This model has also been interpreted as a cross-correlation model [125]. It may be expressed as

$$
h(d)=e^{-d / d_{0}},
$$

where $d_{0}>0$ is the tunable parameter called the decorrelation distance (sometimes defined for $50 \%$ correlation, rather than $1 / e$ ). Literature surveys have been made $[10,134]$ of the values that $d_{0}$ might take in field measurements. While this model can be understood as being based on a first-order auto-regressive $(\operatorname{AR}(1))$, i.e., first-order Markov, process [103], its positive semidefiniteness is not thereby evident, since, on a non-linear trajectory, non-successive points may have different correlation coefficients than simply those constructed by an $\mathrm{AR}(1)$ filter. The fact that $e^{-x / d_{0}}$ is a psd function on $\mathbb{R}^{+}$is a necessary but not sufficient condition [56, p.361] (the same applies for any model depending on $d$ ). Nevertheless, this model is proved to be psd using Theorem 6 in [56, p.362], and similarly in [57].

For equally-spaced ordered points $Y_{1}, \ldots, Y_{N}$ on a straight line with separation $d_{\text {sep }}$, we have the correlation coefficients equal to $\rho_{\imath, \jmath}=\rho^{|\imath-\jmath|}$, where $\rho=e^{-d_{\text {sep }} / d_{0}}$. We then have a correlation matrix as in [137], which is necessarily psd, because the model (2.15) is psd. 


\subsubsection{More Complex Models}

The exponential model has inspired some more complex models, which may be interpreted as auto-or cross-correlation models.

In $[88,92,138]$, a sum of two independent exponential processes is used, which leads to the following correlation model:

$$
h(d)=a e^{-d / d_{1}}+(1-a) e^{-d / d_{2}},
$$

where $0 \leq a \leq 1,0<d_{1}, 0<d_{2}$ are tunable parameters. This model is always psd.

Proof. Applying (2.16) in (2.5), we may separate the resulting integral by linearity into two integrals, both of which are non-negative as seen for the model (2.15), and weighed by the non-negative coefficients $a$ and $1-a$. The resulting integral (2.5) for this model is thus non-negative. It follows from Theorem 6 that this model is psd.

In $[117,129]$ (and implicitly in [131]), a Gaussian correlation model is used:

$$
h(d)=e^{-(d / \bar{d})^{2}}
$$

where $\bar{d}>0$ is the tunable parameter. This model is proved to be psd using Theorem 6 in $[56, \mathrm{p} .364]$.

In [131], a convolution of an exponential and a Gaussian function is proposed:

$$
h(d)=K e^{-|d| / d_{0}} * e^{-(d / \bar{d})^{2}}=K \int_{-\infty}^{\infty} e^{-|d-t| / d_{0}-t^{2} / \bar{d}^{2}} d t
$$

where $d_{0}>0, \bar{d}>0$ are the tunable parameters, and $K=\exp \left(-\bar{d}^{2} / 4 d_{0}^{2}\right) / \sqrt{\pi} \bar{d}$. This model is psd. 
Proof. The Fourier transform of (2.18) with $x=d$ is

$$
f(\omega)=K \frac{2 / d_{0}}{\omega^{2}+d_{0}^{-2}} \bar{d} \sqrt{\pi} e^{-\frac{1}{4}(\bar{d} \omega)^{2}},
$$

which meets the conditions of Theorem 7 .

In [42], a model is proposed that can be written:

$$
h(d)=e^{-\left(d / d_{0}\right)^{\nu}},
$$

with $d_{0}>0, \nu>0$ tunable parameters. The positive semidefiniteness of this model is dependent on $\nu$ : for $0<\nu \leq 2$, it is proved to be psd with the aid of Theorem 7 in [56, p.364]. This of course includes the models (2.15) and (2.17), as well as the choice of $\nu=0.9682$ in [42]. For $\nu>2$ however, the model is in non-psd [56, p.137]. As a counterexample, consider three aligned points with equal consecutive spacings of $0.2 d_{0}$, for which we find that the correlation matrix is not psd for $\nu=$ $2.1,2.2,2.5,3,5,50$.

\subsubsection{Angle-Only Models}

Some of the first shadowing correlation measurements along $\theta$ were reported in [79, 85], but no analytical model was extracted. A similar measurement campaign [97] reported much lower angular correlation, suggesting the need for a more complex model. However, it has been argued [103] that $\theta$ is the most significant parameter in cross-correlation, which justifies using these models as a first approximation.

In $[112,133]$, and later in $[76,139]$, a cosine model was proposed:

$$
h(\theta)=A \cos \theta+B,
$$


with two tunable parameters $A \geq 0, B \geq 0, A+B \leq 1$. The model was used and assumed psd in [103]. Typical parameter choices have been $A=0.3, B=0.5$ $[78,80,82,118]$ and $A=0.3, B=0.699(9)[76,139]$.

The model was proved to be psd in $[57,69,112]$ using Theorem 8 , by setting $a_{0}=A, a_{1}=B$ and all other $a_{n}=0$.

In [94], a piecewise--linear model was proposed:

$$
h(\theta)= \begin{cases}0.78-7 \theta / 1250^{\circ} & 0^{\circ} \leq \theta<15^{\circ}, \\ 0.48-7 \theta / 1250^{\circ} & 15^{\circ} \leq \theta<60^{\circ}, \\ 0 & 60^{\circ} \leq \theta \leq 180^{\circ} .\end{cases}
$$

It was shown in [113] that this model is non-psd. Here is an example: $N=7$ with $\angle \vec{r}_{\imath}=0,5, \ldots, 30^{\circ}$.

In [93, Table 1], a lookup table for intervals of $\theta$, effectively a piecewise-constant model was given:

$$
h(\theta)= \begin{cases}0.6 & 0^{\circ} \leq \theta<30^{\circ} \\ 0.25 & 30^{\circ}<\theta<60^{\circ} \\ \alpha \geq 0.2 & \theta \geq 90^{\circ}\end{cases}
$$

This model is undefined for $\theta \in\left\{30^{\circ}\right\} \cup\left[60^{\circ}, 90^{\circ}\right]$. We understand that a value for $\alpha$ may be chosen on $[0.2,0.25]$. Regardless of the missing information, the model is non-psd, as shown in this example: $N=11$ with $\angle \vec{r}_{\imath}=0,4, \ldots, 40^{\circ}$.

A triangular model in $\theta$ was proposed in [48]:

$$
h(\theta)= \begin{cases}a-(a-b) \theta / \theta_{0} & \theta \leq \theta_{0} \\ b & \theta>\theta_{0}\end{cases}
$$

with $0 \leq b<a \leq 1$ and $0^{\circ}<\theta_{0} \leq 180^{\circ}$ three tunable parameters. In [48], the model 
$a=0.8, b=0.4, \theta_{0}=60^{\circ}$ was used to fit the measurements in [85]. The same choice of parameters was proposed for cross-correlation modeling for the $802.16 \mathrm{~m}$ standard [135]. Also, $a=0.8, b=0, \theta_{0}=60^{\circ}$ was proposed to fit the measurements in [94], previously fitted with (2.21). In [88], the same model with $a=0.9, b=0, \theta_{0}=180^{\circ}$ was used. This model is always psd for any choice of parameters.

Proof. Consider

$$
a_{0}=b+(a-b) \frac{\theta_{0}}{2 \pi}, \quad a_{n}=2(a-b) \frac{1-\cos \theta_{0} n}{\pi \theta_{0} n^{2}} .
$$

Using Theorem 8 yields (2.23).

A decaying exponential angle model has also been proposed $[57,67,101,140]$ :

$$
h(\theta)=e^{-\alpha \theta}
$$

with $\alpha>0$ a tunable parameter. This model can be seen as inspired from the exponential $d$ model (2.15), which it approximates for small $\theta$. This model is psd [57].

Proof. Consider

$$
a_{0}=\frac{1-e^{-\alpha \pi}}{\alpha \pi}, \quad a_{n}=\frac{2}{\pi} \frac{\alpha}{n^{2}+\alpha^{2}}\left(1-e^{-\alpha \pi}(-1)^{n}\right)
$$

Using Theorem 8 yields (2.24).

\subsubsection{Separable Models}

Separable models are easily constructed from the multiplication of one dimensional models. Theorem 10 shows that if the component one-dimensional models are psd, so is the composite separable model. 


\subsubsection{Angle-Distance Ratio}

Separable $\theta-R$ models may always be written as

$$
h(\theta, R)=h_{\Theta}(\theta) h_{\mathrm{R}}(R) .
$$

The use of the $\theta$ and $R$ dimensions for shadowing correlation models has been argued in $[48,57,97,98,103,118]$, though separability may be a simplistic assumption [57]. In [48], the model "1.0/0.0 RX" is given as

$$
h_{\Theta}(\theta)= \begin{cases}1-\theta / 75^{\circ} & \theta \leq 60^{\circ} \\ 0 & \theta \leq 60^{\circ}\end{cases}
$$

and

$$
h_{\mathrm{R}}(R)=\max \left(0,1-R / R_{0}\right)
$$

with $R_{0}>0$, with $R_{0}$ usually in $[6 \mathrm{~dB}, 20 \mathrm{~dB}]$. This model is not psd, as shown in the following example: let $N=7$ and all $r_{\imath}$ equal, and let $\vec{r}_{\imath}=0,15, \ldots, 90^{\circ}$.

The problem with the preceding model is its discontinuity at $\theta=60^{\circ}$. This is simple to resolve: if we use the general form (2.23) as $h_{\Theta}(\theta)$ and (2.27) as $h_{\mathrm{R}}(R)$, we have a very flexible model that is always psd.

Proof. $h_{\mathrm{R}}(R)$ is shown to be psd by choosing

$$
f(\omega)=\frac{2 \sin ^{2}\left(\pi R_{0} \omega\right)}{\pi^{2} R_{0} \omega^{2}}
$$

with $g(\vec{r})=\frac{10}{\ln 10} \ln \|\vec{r}\|$ in Theorem 9. Equivalently, we may use Pólya's Theorem, as in $[57]$.

The model (2.23) was already shown to be psd.

Now $h$ is expressed as a product of two psd models, and it follows from Theorem 
10 that $h(\theta, R)$ is psd.

\subsubsection{Angle-Absolute Distance}

In [104], a separable model depending on $d$ and $\theta$ is used, and can be rewritten:

$$
\begin{gathered}
h_{\mathrm{d}}(d)=e^{-d / d_{0}}, \\
h_{\Theta}(\theta)= \begin{cases}1 & \theta \leq 90^{\circ}, \\
0 & \theta>90^{\circ},\end{cases} \\
h(d, \theta)=h_{\mathrm{d}}(d) h_{\Theta}(\theta) .
\end{gathered}
$$

(There is a mistype in the original equation [104, eq. 18]: the term $\sigma_{\bar{\psi}_{p}}^{2}$ should be removed, as confirmed in a private communication with D. Kaltakis).

The authors say that this model is psd; however, this claim is not substantiated, though we understand that their simulations always gave psd correlation matrices. We already recognise the model $h_{\mathrm{d}}(d)$ from (2.15), which we know to be psd. However, it is simple to show that $h_{\Theta}$ is non-psd, therefore the product of the two might not be psd either. In fact, the following counterexample shows it is not psd: $N=14$ with $r_{\imath}=d_{0}, \angle \vec{r}_{\imath}=0,180 / 7, \ldots, 2340 / 7^{\circ}$.

The authors of [104] claim that this model is psd, based on their simulations. This is to be expected: because the model can be approximated by (2.15) for $r_{\imath} \gg d_{0}$, as might be the case in the cellular context, it can often appear psd in simulations. However, strictly speaking, it is non-psd, and our example shows that it can fail, particularly in cases of small $r_{\imath}$. 
In [141], the same author proposes

$$
\begin{aligned}
h_{\mathrm{d}}(d) & =e^{-d / d_{0}} \\
h_{\Theta}(\theta) & =\max (0, \cos (\theta)) \\
h(d, \theta) & =h_{\mathrm{d}}(d) h_{\Theta}(\theta) .
\end{aligned}
$$

Again, while the model appears psd [141], in fact it is not, as attested by the following counterexample: $N=8$ with $r_{\imath}=0.4 d_{0}, \angle \vec{r}_{\imath}=0,45, \ldots, 325^{\circ}$. However, this is a case with very small $r_{2}$. In practice, for $r_{2} \gg d_{0}$, this model is well-approximated by

$$
h(d, \theta)=e^{-d / d_{0}} \cos (\theta),
$$

which is psd.

Proof. Model (2.30) is the product of models (2.15) and (2.20) with $A=1, B=0$, both of which were shown to be psd. Then, by Theorem 10, (2.30) is psd.

\subsubsection{More Elaborate (Non-Separable) Models}

It has been suggested [57] that separable models might not be sufficient to accurately model shadowing correlation.

Saunders' model [124] has been used in $[70,102,103,113,142]$ (but watch for various transcription errors):

$$
\begin{aligned}
& h_{1}=10^{-005 R}, \quad h_{2}= \begin{cases}1 & \theta<\theta_{\mathrm{T}}, \\
\left(\theta_{\mathrm{T}} / \theta\right)^{\gamma} & \theta \geq \theta_{\mathrm{T}},\end{cases} \\
& \theta_{\mathrm{T}}=2 \arcsin \frac{d_{0}}{2 \min \left\{r_{1}, r_{2}\right\}}, \quad h\left(\theta, r_{1}, r_{2}\right)=h_{1} h_{2},
\end{aligned}
$$

where $d_{0}$ is the same correlation distance as measured for model (2.15), and $\gamma>0$ is 
the other tunable parameter (a typical value is $\gamma=0.3$ ). The model is not separable in $\theta$ and $R$ because $\theta_{\mathrm{T}}$ depends on $r_{1}$ and $r_{2}$. This model is non-psd, as seen in [103,104,113] and as is apparent from this example: $N=3$ with $r_{i}=r \geq d_{0} / \sqrt{2}$ and $\angle \vec{r}_{\imath}=0, \theta_{\mathrm{T}}, 2 \theta_{\mathrm{T}}$.

Also, we see that this model is undefined for $r_{2}<d_{0} / 2$, since the domain of arcsin is $[-1,1]$. To rectify this problem, Saunders' model has been modified [66] (also [136], but note some transcription errors) by augmenting its domain:

$$
h\left(\theta, r_{1}, r_{2}\right)= \begin{cases}\text { as in }(2.31) & r_{1}, r_{2} \geq d_{0} / 2 \\ \sqrt{d_{0} / 2 \max \left\{r_{1}, r_{2}\right\}} & \text { otherwise }\end{cases}
$$

Notice however that if more than one $r_{\imath}$ is less than $d_{0} / 2$, we again have $h>1$, which is not a valid correlation value.

Also, extending the model's domain cannot make it psd: the same counterexamples used to show that (2.31) is non-psd can be used to show that (2.32) is non-psd.

Another model was given in [93, Table 2] and used in [103]. It is piecewise constant on rectangles in the $\theta-R$ domain:

$$
h(\theta, R)=\left\{\begin{array}{cccc}
R<2 & 2<R<4 & R \geq 4 \\
0.8 & 0.6 & 0.4 & \theta<30^{\circ}, \\
0.5 & 0.4 & 0.2 & 30^{\circ}<\theta<60^{\circ}, \\
0.4 & 0.4 & 0.2 & 60^{\circ}<\theta<90^{\circ}, \\
0.2 & 0.2 & 0.2 & \theta \geq 90^{\circ} .
\end{array}\right.
$$

The entries of this table cannot be obtained from an outer product, and thus the model is non-separable. The model is non-psd, as observed in [103, 104, 113]; for example: $N=6$ with $r_{i}=r \forall i$ and $\angle \vec{r}_{\imath}=0,7,14,21,28,35^{\circ}$. 
Another model is given in [83]:

$$
\begin{gathered}
h_{\Theta}(\theta) \quad \text { as in }(2.21), \\
h_{\mathrm{R}}(R)=\max \left(0,\left(1-R / R_{0}\right)^{\alpha}\right) \\
h(\theta, R)=h_{\mathrm{R}}(R)\left(h_{\Theta}(\theta)+a\right)+b,
\end{gathered}
$$

with $a>0, b>0, a+b \leq 0.22, \alpha \geq 0$ and $R_{0}>0$. When $b \neq 0$, this model is not quite separable. It is not psd, as seen from the same counterexample as used for model (2.21), with $a=b=0$ and $r_{\imath}=r \forall i$.

Finally, the model "1.0/0.4 RX" in [48] takes the form:

$$
h(\theta, R)= \begin{cases}\max \left(0,1-\frac{R}{R_{0}}\right)\left(0.6-\frac{\theta}{150^{\circ}}\right)+0.4 & \theta \leq 60^{\circ} \\ 0.4 & \theta>60^{\circ}\end{cases}
$$

where $R_{0}$ is chosen between 6 and $20 \mathrm{~dB}$. This model is not psd, as attested by the following example: with $N=4$, regardless of the value of $R_{0}$, choose $r_{2}$ all equal, and $\angle \vec{r}_{\imath}=0,30,60,90^{\circ}$

\subsection{Some Thoughts on Physical Realism}

While this chapter focuses on the mathematical feasibility of correlation models, we would like to give a few thoughts about physical realism as well: can the correlation model $h$ be considered realistic, based on what we know about wireless propagation? Does it make sense intuitively? It is important to keep in mind that, while mathematical feasibility is an objective criterion, physical plausibility is relative to the understanding of wireless shadowing, and therefore the criteria presented in this section are merely tentative. We hope that these initial ideas will encourage thought and discussion on what a shadowing correlation model should realistically look like. 
Table 2.1: Summary of existing shadowing correlation models and their properties.

\begin{tabular}{|c|c|c|c|c|c|c|}
\hline Model Name & Equation & $\begin{array}{l}\text { Tunable Parame- } \\
\text { ters }\end{array}$ & References & Dimensions & psd & $\begin{array}{l}\text { Physical Proper- } \\
\text { ties }^{b} \text { Violated }\end{array}$ \\
\hline Constant & $(214)$ & $0<\rho<1$ & many & none & Yes & $1,5,7$ \\
\hline $\operatorname{Exp}(d)$ & $(215)$ & $d_{0}>0$ & {$[43,86]$} & $d$ & Yes & 7 \\
\hline $\operatorname{Exp}+\operatorname{Exp}(d)$ & $(216)$ & $\begin{array}{l}0 \leq a \leq 1,0< \\
d_{1}, 0<d_{2}\end{array}$ & {$[88,92,138]$} & $d$ & Yes & 7 \\
\hline $\operatorname{Gaussian}(d)$ & $\left(\begin{array}{ll}2 & 17\end{array}\right)$ & $\vec{d}>0$ & {$[129]$} & $d$ & Yes & 7 \\
\hline $\operatorname{Exp} * \operatorname{Gaussian}(d)$ & $\left(\begin{array}{ll}2 & 18\end{array}\right)$ & $d_{0}>0, \bar{d}>0$ & [131] & $d$ & Yes & 7 \\
\hline \multirow[t]{2}{*}{$\operatorname{Exp}\left(d^{\nu}\right)$} & $\left(\begin{array}{ll}2 & 19\end{array}\right)$ & $d_{0}>0,0<\nu \leq 2$ & {$[42]$} & $d$ & Yes & 7 \\
\hline & & $d_{0}>0, \nu>2$ & & & No & 7 \\
\hline $\operatorname{Cos}(\theta)$ & $(220)$ & $\begin{array}{l}A \geq 0, B \geq 0, A+ \\
B \leq 1\end{array}$ & {$[76,112,133,139]$} & $\theta$ & Yes & $(1)^{c},(2)^{c}, 4,(5)^{c}$ \\
\hline Plecewise $(\theta)$ & $\left(\begin{array}{l}2 \\
21\end{array}\right)$ & none & {$[94]$} & $\theta$ & No & 1,6 \\
\hline Stepwise $(\theta)$ & $(222)$ & none & {$[93$, Table 1] } & $\theta$ & No & $1,5,6$ \\
\hline Trıangular $(\theta)$ & $\left(\begin{array}{ll}2 & 23\end{array}\right)$ & $\begin{array}{l}0 \leq b<a \leq 1,0^{\circ}< \\
\theta_{0}<180^{\circ}\end{array}$ & {$[48]$} & $\theta$ & Yes & $(1)^{c},(2)^{c},(5)^{c}$ \\
\hline $\operatorname{Exp}(\theta)$ & $(224)$ & $\alpha>0$ & {$[57,67,101,140]$} & $\theta$ & Yes & $2,(5)^{c}$ \\
\hline "1 0/0 0 RX" & $\begin{array}{l}\left(\begin{array}{ll}2 & 25\end{array}\right) \\
(25) \\
(25)\end{array}$ & $R_{0}>0$ & {$[48]$} & $\theta, R$ & No & 6 \\
\hline \multirow[t]{2}{*}{$\begin{array}{l}\text { Trıangular }(\theta) \times \\
\text { Trıangular }(R)\end{array}$} & 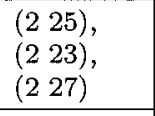 & $\begin{array}{l}0 \leq b<a \leq 1 \\
0^{\circ}<\theta_{0}<180^{\circ} \\
R_{0}>0\end{array}$ & modified [48] & $\theta, R$ & Yes & $(1)^{c},(5)^{c}$ \\
\hline & & $b=0, a=1$ & & & Yes & none \\
\hline $\operatorname{Exp}(d) \times\left(\theta \leq 90^{\circ}\right)$ & $(228)$ & $d_{0}>0$ & [104] & $d, \theta$ & No & 6 \\
\hline $\operatorname{Exp}(d) \times \cos ^{+}(\theta)$ & $(229)$ & $d_{0}>0$ & [141] & $d, \theta$ & No & none \\
\hline $\operatorname{Exp}(d) \times \cos (\theta)$ & $(230)$ & $d_{0}>0$ & modified [141] & $d, \theta$ & Yes & 4 \\
\hline Saunders' & $\left(\begin{array}{l}2 \\
31\end{array}\right)$ & $d_{0}>0, \gamma>0$ & {$[124,143]$} & $\begin{array}{l}\left(\theta, r_{1}, r_{2}\right)^{a} \\
r_{\imath}>d_{0} / 2\end{array}$ & No & $2,(5)^{c}$ \\
\hline $\begin{array}{l}\text { Modified Saun- } \\
\text { ders' }\end{array}$ & $\left(\begin{array}{l}2 \\
32\end{array}\right)$ & $d_{0}>0, \gamma>0$ & {$[66]$} & $\begin{array}{l}\left(\theta, r_{1}, r_{2}\right)^{a} \\
r_{2}, \quad, r_{N}> \\
d_{0} / 2\end{array}$ & No & $2,3,(5)^{c}, 6$ \\
\hline Stepwise $(\theta, R)$ & $(233)$ & none & [93, Table 2] & $(\theta, R)^{a}$ & No & $1,5,6$ \\
\hline $\begin{array}{l}\text { Plecewise }(\theta)- \\
\operatorname{Tr} \text { iangular } \\
\end{array}$ & $(234)$ & $\begin{array}{l}R_{0}>0, \alpha \geq 0, a> \\
0, b>0, a+b \leq 022\end{array}$ & {$[83]$} & $(\theta, R)^{a}$ & No & $(1)^{c},(5)^{c}, 6$ \\
\hline "1 0/0 4 RX" & $(235)$ & $R_{0}>0$ & {$[48]$} & $(\theta, R)^{a}$ & No & 5,6 \\
\hline
\end{tabular}

${ }^{a}$ Non-separable

${ }^{b}$ As described in Section 2.5

${ }^{c}$ Physical property met only for some parameter choices

Nevertheless, these initial criteria can give a first approximation in choosing a good correlation model.

Proposition 1. Shadowing is a large-scale phenomenon, averaged over small displacements in space (and time), and should not by nature vary quickly in these 
dimensions $[43,57,103,118]$.

Proposition 2. As argued in $[10,48,70,79,97,115,116,124,142,143]$, correlation in shadowing may be explained by a partial overlap of the large-scale propagation medium, as seen in Figure 2.5. The non-overlapping propagation areas are considered independent. When a propagation front passes through successive areas, the gains are multiplicative, and thus add in the logarithmic domain. We may then write:

Let $W, W_{1}, W_{2}$ be independent $\mathrm{RVs}$ with zero mean and variances $w^{2}, w_{1}^{2}, w_{2}^{2}$ respectively. We have

$$
\begin{aligned}
& S_{1}=W+W_{1} \\
& S_{2}=W+W_{2}
\end{aligned}
$$

It follows that

$$
\begin{aligned}
& \operatorname{VAR}\left\{S_{1}\right\}=w^{2}+w_{1}^{2}=\sigma_{\mathrm{s}}^{2}\left(\vec{r}_{1}\right), \\
& \operatorname{VAR}\left\{S_{2}\right\}=w^{2}+w_{2}^{2}=\sigma_{\mathrm{s}}^{2}\left(\vec{r}_{2}\right), \\
& \mathbb{E}\left\{S_{1} S_{2}\right\}=w^{2},
\end{aligned}
$$

and therefore

$$
h\left(\vec{r}_{1}, \vec{r}_{2}\right)=\frac{w^{2}}{\sqrt{\left(w^{2}+w_{1}^{2}\right)\left(w^{2}+w_{2}^{2}\right)}} .
$$

This applies to any pair of shadowing terms.

This interpretation leads us to formulate the following criteria:

1. $h\left(\vec{r}_{\imath}, \vec{r}_{\jmath}\right) \approx 1$ for $\vec{r}_{\imath} \approx \vec{r}_{\jmath}$.

2. $h\left(\vec{r}_{\imath}, \vec{r}_{\jmath}\right) \ll 1$ for $\left\|\vec{r}_{\imath}-\vec{r}_{\jmath}\right\| \gg 0$.

3. $h$ should be a non-increasing function in $\theta[57,85,93,103], R[93]$ and $d$.

4. $h$ should be non-negative, according to (2.37). However, this is contradicted by some measurement campaigns [43, 85, 87, 90,94,96-99], which reported some significantly negative estimated correlation coefficients in some cases. It would 


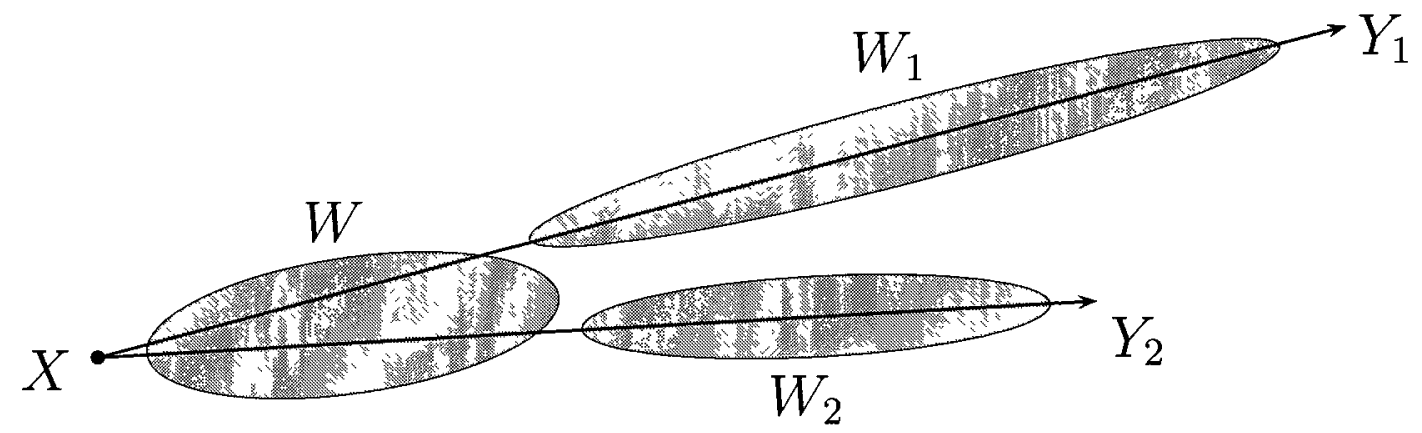

Figure 2.5: Physical argument of common propagation area for correlation in shadowing.

be interesting to study whether these observations are statistically significant. Also, we suspect that negative correlations in measurements may appear in a particular obstacle scenario. However, averaged over very many obstacle realisations, the correlations are perhaps less likely to be negative.

5. $h$ should be small for large $\theta$, and approach zero for $\theta \approx 180^{\circ}$ and $r_{1}, r_{2}$ large, as the propagation regions are then essentially non-overlapping.

6. Continuity: a small change in $\vec{r}_{i}$ should result in small changes in $h\left(\vec{r}_{i}, \vec{r}_{j}\right)$, at least when $r_{i}$ is large.

7. Correlation should not depend on $d$ only, as the distance between $\vec{r}_{1}$ and $\vec{r}_{2}$ tells us little about the overlap between the corresponding propagation areas.

Furthermore, it was argued $[48,57,97,98,103,118]$ that cross-correlation should depend on $\theta$ and $R$.

We have checked each model against these physical constraints, and summarised the results (without proof) in Table 2.1. The only model that is both psd and fulfills all these physical criteria is the one we proposed based on [48], given by $(2.23),(2.25)$, and (2.27), with $a=1, b=0$. 


\subsection{Feasibility According to Marginal Distribution}

Until now, we have only verified whether a given model $h$ gives psd covariance matrices $\mathbf{K}$. This is a necessary but not always sufficient condition to ensure that a random vector $\vec{S}$ can be constructed with covariance K. To further study feasibility, it is necessary to look at the marginal distribution of $S_{\imath}$.

\subsubsection{Jointly Lognormal Shadowing}

In the most common case of lognormal shadowing (i.e., each $S_{\imath}$ being Gaussian), a natural and effective way to model shadowing paths jointly is by making $S_{1}, \ldots, S_{N}$ jointly Gaussian. We then say that shadowing is jointly lognormal. In this case, every symmetric psd correlation matrix is feasible, by the following explicit construction:

We begin by solving for $\mathbf{C}_{N \times N}$ in

$$
\mathbf{K}=\mathbf{C C}^{\mathrm{T}} \text {. }
$$

In general, there are many solutions to this equation if and only if $\mathbf{K}$ is psd. Then, we generate an independent column vector of standard Gaussian RVs $\vec{Z}=\left[Z_{1}, \ldots, Z_{N}\right]$, and obtain the shadowing terms with

$$
\vec{S}=\mathrm{C} \vec{Z}
$$

This is a constructive proof that, for lognormal shadowing, feasibility is equivalent to positive semidefiniteness.

\subsubsection{Non-Lognormal Shadowing}

While lognormal is a well-established and by far the most common model for the marginal distribution of shadowing, it is not the only one available. Other models have 
been proposed, either from physical plausibility arguments, or for easier mathematical tractability. The two other models that we have encountered for the distribution of $e^{\lambda S_{\imath}}$ are truncated lognormal $[10,144]$ and Gamma [145-147]. See [10, Section V-B-4] for a survey of shadowing distribution measurements.

We have seen in Section 2.1.4 that the condition of finite variance of $S_{\imath}$ is required for an adequate definition of correlation. For the truncated lognormal model, which corresponds to a truncated Gaussian $S_{\imath}$, it is easy to see that truncation reduces the variance, hence it is finite. For Gamma shadowing, it is easy to verify that all the moments of the logarithm of a Gamma RV are finite, since they can be expressed as the integral 4.352 .1 in [148].

It is, however, not evident that the vector $\vec{S}$ can be generated according to nonGaussian marginal distributions and a given psd correlation matrix K. One constructive method for obtaining such jointly distributed vectors is NORTA (Normal-toAnything), described in [149]. This method requires some more stringent conditions on the marginal distributions to be feasible. These conditions might merit further study should non-lognormal shadowing models gain in popularity.

\subsection{Consequences for Working with Correlated Shadowing}

We propose the criteria developed in this chapter as a basis for evaluating, designing, and correcting existing future correlation models. There is naturally more to be said, both on testing more complex models for being psd and on the physical plausibility of those models. 


\subsubsection{For Those Wanting to Incorporate Shadowing Correlation}

For lognormal shadowing, only certain models that have the psd property (see Table 2.1) guarantee to always give feasible joint shadowing distributions. For nonlognormal shadowing, some additional conditions might be required.

Based on both mathematical feasibility and physical arguments, we conclude that a subset of the family of models given by (3.5) and inspired by [48] is the best existing candidate for modeling correlation in shadowing. With two tunable parameters, $\theta_{0}$ and $R_{0}$, it may be tuned to approximate many other correlation models that might have less desirable properties.

\subsubsection{For Those Using a Model that Is Not Feasible}

Models that are not psd may still be used in particular application scenarios. For example, all models give psd correlation matrices for $N=2$. Also, we have argued that a correlation model that corresponds exactly to reality must per force be psd. It follows that for any model that approximates reality well (whether from extensive measurement campaigns or from theoretical arguments), there exists a model that is close to it (according to some reasonable metric) while also being psd. For example, the authors in [48] fit the psd model (2.23) to the data of [85] to replace the non-psd model (2.21).

Our suggestion is to take non-psd models and correct them slightly to make them psd, as we have done for (2.29).

\subsubsection{For Those Designing New Correlation Models}

We suggest that all new correlation models be designed as psd for the reasons described at the beginning of this chapter. Theorem 10 can be used to construct more detailed separable models. 


\subsubsection{For Those Studying Large Interference Problems}

Finally, a very important specific application of shadowing correlation is the study of the aggregate interference from a large number of devices. The resulting interference distribution differs greatly whether one considers shadowing that is independent, or one that is significantly correlated; this effect becomes more significant as the number of interferers increases. It is this interference distribution that we examine in the rest of the thesis. 


\section{Chapter 3}

\section{Problem Statement}

In this chapter, we specify the quantities and parameters that describe our physical interference problem. Many quantities are random, and these should all be considered fully independent unless specified otherwise. The problem is specified by a description of the wireless channel, of the locations of the ISs and their transmit characteristics, and of the manner in which individual interference signals combine.

\subsection{Interferer Layout}

Since we consider large numbers of wireless (often mobile) ISs, it is natural to position them randomly according to some spatial distribution, rather than give all their positions explicitly $[2,3]$.

We always assume that the RX is located at the origin of the plane, and the positions of the ISs are vectors in 2 dimensions: $\vec{r}_{\imath} \in \mathbb{R}^{2} \backslash\{0\}$, with $r_{\imath}=\left\|\vec{r}_{\imath}\right\|$.

\subsubsection{Poisson Field}

A mathematical model for IS positions that is very simple conceptually is a twodimensional homogeneous Poisson point process (PPP) $[4,25,150]$, sometimes called a Poisson field. It is characterised simply by a scalar density $\lambda_{\mathrm{P}}$. It can be simply 
defined by stating that for any Borel region $\mathcal{A}$ of finite Lebesgue measure (area) $|\mathcal{A}|$, the number of ISs inside $\mathcal{A}$ follows a Poisson distribution with mean $\lambda_{\mathrm{P}}|\mathcal{A}|$. Furthermore, for any set of such disjoint areas, the numbers of points lying inside those areas are independent [150].

Equivalently, we may construct such a process by considering a circular region of radius $r_{\max }$ centered at the origin, and generating $N$ iid uniformly-distributed points $\vec{r}_{\imath}$ on the disc, where $N$ is a Poisson RV with mean $\pi r_{\max }^{2} \lambda_{\mathrm{P}}$, then taking $r_{\max } \rightarrow \infty$. For simulation purposes, it is necessary to limit $r_{\max }$ to a large finite value.

In [150], a finite area of ISs is considered, modeled as a Bernoulli Point Process, which is somewhat more general, but still very restrictive. These models are the starting point for our thinking about layouts. Still, we eschew the above two models in favour of a much more general layout described by a weighted spatial distribution.

\subsubsection{Weighed Spatial Distribution}

A much more generalised approach [32] is to sample the positions $\vec{r}_{\imath}$ from a 2dimensional pdf $g(\vec{r})$, i.e., for any Borel set $\mathcal{A}$ in $\mathbb{R}^{2}$, we have $\mathbb{P}\left(\vec{r}_{\imath} \in \mathcal{A}\right)=\int_{\mathcal{A}} g(\vec{r}) d \vec{r}$. It is also convenient to define $\mathcal{A}_{g}$ the entire area where $g$ is non-zero, and $r_{\min }, r_{\max }$, the tightest bounds such that $g(\vec{r})=0$ for $r$ outside $\left[r_{\min }, r_{\max }\right]$. The interfering network is then composed of $N$ ISs with positions chosen iid from $g(\vec{r})$. In this thesis, we consider $N$ to be a non-random quantity. However, in Section 6.2.1 we describe how to modify our work to incorporate a random $N$, e.g., from a Poisson distribution.

This is a quite general formulation of the problem and can include many conceivable scenarios. Still, it cannot model particular behaviours, such as thinning, clustering, or other phenomena between the ISs that require dependence among the IS positions $[151,152]$. We do not consider such effects in this thesis, but show in Chapter 6.2.2 how they may be incorporated. 


\subsubsection{Cluster Layouts}

As a subset of the generalised spatial distribution $g(\vec{r})$ just given, we take special interest in those that exhibit what we call a cluster geometry. We do not give an exact definition of a cluster layout. Rather, as a rule of thumb, we suggest that a cluster of interferers is one where (almost) all the ISs are seen at a fairly narrow angle from the RX (not much more than a $60^{\circ}$ spread), and that the cluster of ISs has roughly circular symmetry, as a consequence of which the spread of the distances between the RX and the ISs is not too significant (not much more than 4 to 1 for farthest to nearest).

A cluster layout generally applies in the case where the RX and the ISs do not belong to the same network, simply because of the nature of such a geometry. The cluster layout is of interest to us because it is only in this case that we find a simple mostly analytical solution.

\subsubsection{Layout Examples}

In this thesis, we use four specific layouts, which we illustrate in Figure 3.1:

A A uniform distribution over a square region away from the RX. This is an example of a cluster layout.

B A circular Gaussian distribution truncated at four standard deviations over a circular region away from the RX. This is another cluster layout.

C An annular region centred around the RX. There is a relatively small exclusion region around the RX to prevent problems with ISs arbitrarily close to the RX. This type of layout is usually assumed (with possibly the outer radius going to infinity, and possibly the inner radius zero) in the literature described in 1.2.2.

D A very thin annular region centred around the RX. This is not really a likely 

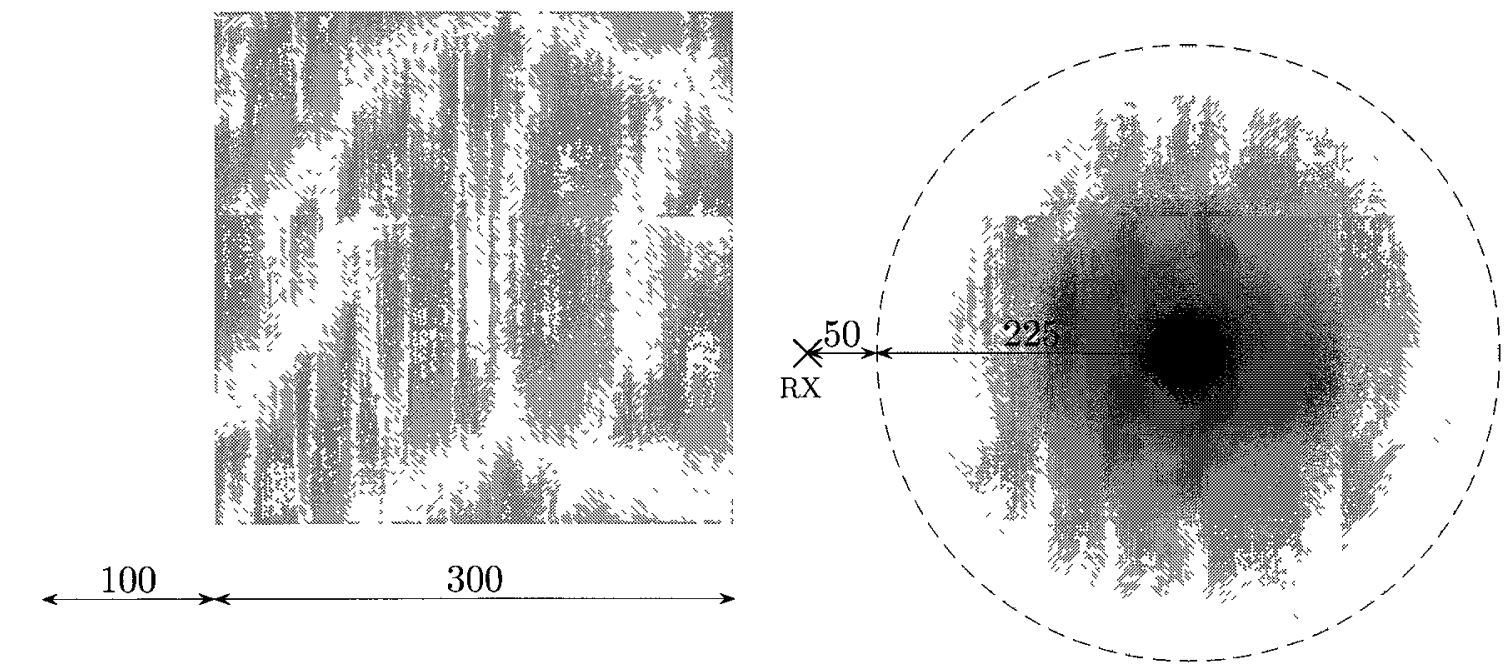

$\underset{\mathrm{RX}}{\mathrm{X}} g(x, y)= \begin{cases}\frac{1}{9} \cdot 10^{-4} & 100<x<400 \\ & 100<y<400 \\ 0 & \text { otherwise }\end{cases}$

A : Square Cluster

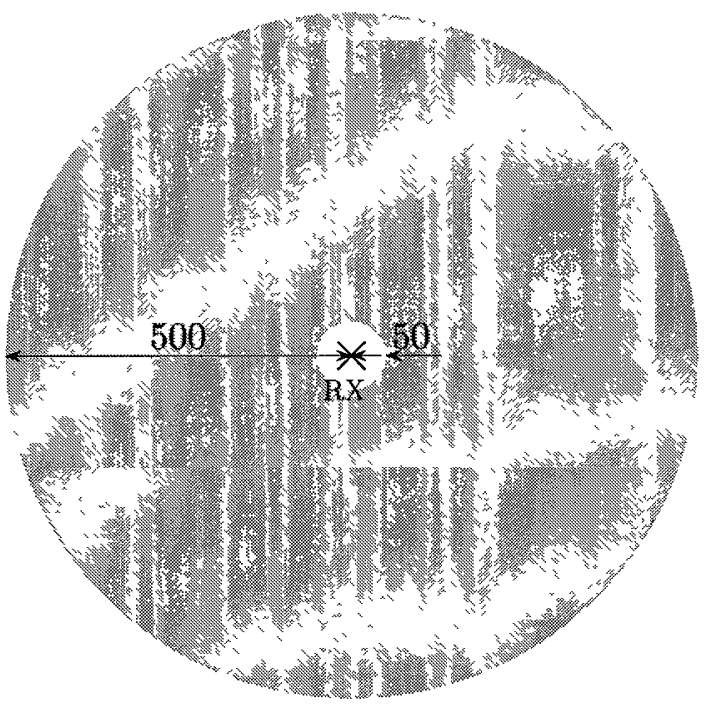

$g(\vec{r})= \begin{cases}(247500 \pi)^{-1} & 50<r<500 \\ 0 & \text { otherwise }\end{cases}$ $g(\vec{r})= \begin{cases}\frac{e^{-\frac{\left\|\vec{r}-\vec{r}_{c}\right\|^{2}}{225^{2} / 8}}}{\left(1-e^{-4}\right) 225^{2} \pi / 8} & \left\|\vec{r}-\vec{r}_{0}\right\|<225 \\ 0 & \text { otherwise }\end{cases}$ where $\vec{r}_{\mathrm{c}}=(275,0)$

\section{B : Gaussian Cluster}
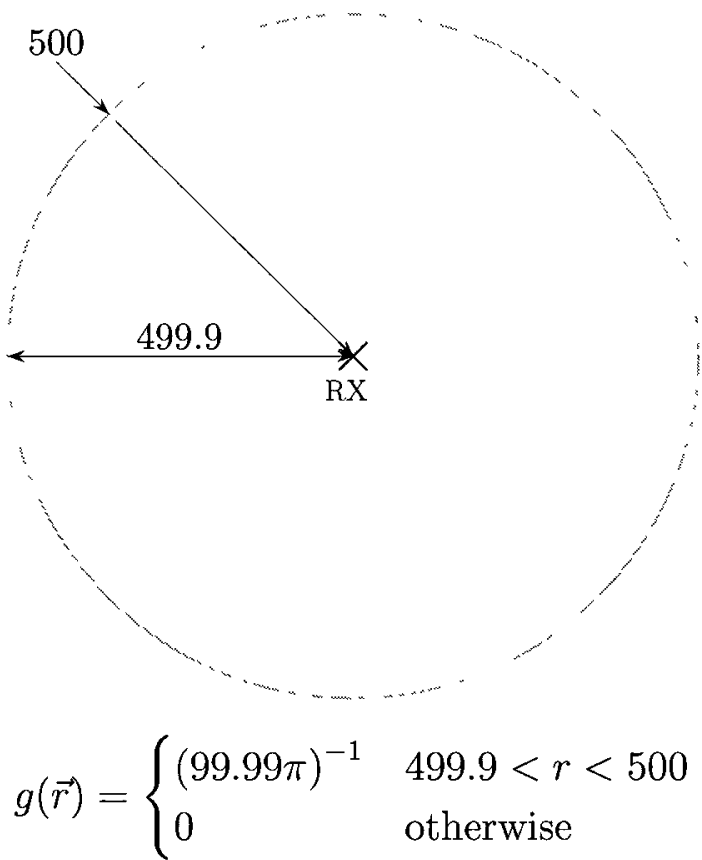

D : Surrounding Thin Ring

Figure 3.1: Interferer layouts used in this thesis. Distances are in meters. 
real world scenario. Instead, we only use it to separate the effect of shadowing from pathloss in order to better understand the effects of each. Indeed, in this layout, $r$, and thus the pathloss, is almost constant.

\subsection{Channel Models}

In this section, we define the propagation channel between two points, as well as the correlation between two paths with one common point. We are interested exclusively in large-scale outdoor propagation scenarios. Also, we consider a single frequency channel at a single moment; there is thus no modeling across frequency or time (we discuss this issue briefly in Section 6.2.5). We consider pathloss, shadowing, and shadowing correlation, but not small-scale fading. In Section 6.2.3, we discuss how small-scale fading can be incorporated into our results without much effort.

Various components come together to make up the channel model between a transmitter and a receiver. These are modeled as RVs, whose statistics are known either through estimation based on empirical measurements in the field, or through physical arguments.

\subsubsection{Average Pathloss}

The average pathloss is a deterministic function of distance, which we denote $p(r)$. Pathloss is often $[4,37,57]$ modeled as power-law: $p(r)=r^{-\beta}$ for $2 \leq \beta \leq 6$. However, to allow for the incorporation of both very near and very far-away interferers, more flexibility in the model is useful.

To this end, we use a model that behaves similarly to free space $(\beta=2)$ before the Fresnel breakpoint and according to a two ray model $(\beta=4)$ for larger distances 
[153]. The model is based on [154] and is given by

$$
p(r)=r^{-2}(1+r / 150)^{-2} .
$$

The distances are in meters, and we see the breakpoint around 150 meters, with the added elegance of a smooth transition. This model is taken as a realistic example only and does not restrict the scope of our work.

\subsubsection{Lognormal Shadowing}

The lognormal distribution is a well established $[10,71,87,124,128]$ model for largescale wireless fading, i.e., shadowing. The shadowing gain (in $\mathrm{dB}$ ) between an IS $i$

and the RX is then given by a Gaussian RV $S_{i}$ with mean 0 , and variance $\sigma_{\mathrm{s}}^{2}(r)$ a function of distance. The power on that path is then scaled by a factor of $e^{\lambda S_{i}}$, with $\lambda=0.1 \ln 10$.

While shadowing is often found to have approximately constant spread $\sigma_{\mathrm{s}}$ over distance, this may not be the case for very short distances. Indeed we may use the following model, taken from [66]

$$
\sigma_{\mathrm{s}}(r)=10(1-\exp (-3 r / 200)) \mathrm{dB},
$$

where the distances are in meters. We see that the shadowing spread stays within $5 \%$ of $10 \mathrm{~dB}$ for distances above 200 meters. Again, this is just a useful example of a model to illustrate our work.

\subsubsection{Spatially-Correlated Jointly Lognormal Shadowing}

Of great importance to this work is the joint distribution of the shadowing paths. While little is known about the exact nature of this distribution, the most common 
(in fact, perhaps the only) approach is to consider the shadowing paths as jointly lognormal (when conditioned on position). This approach could be said to be the most natural to formulate and to use, and goes as follows:

We construct a vector $\vec{S}=\left[S_{\imath}\right]_{\imath=1}^{N}$ that is (jointly) Gaussian when conditioned on $\vec{r}_{1}, \ldots, \vec{r}_{N}$. The vector is fully statistically described by the quantities

$$
\begin{aligned}
\mathbb{E}\left\{S_{\imath}\right\} & =0, \\
\mathbb{E}\left\{S_{\imath}^{2} \mid r_{\imath}\right\} & =\sigma_{\mathrm{s}}^{2}\left(r_{\imath}\right), \\
\mathbb{E}\left\{S_{\imath} S_{\jmath} \mid \vec{r}_{\imath}, \vec{r}_{\jmath}\right\} & =\sigma_{\mathrm{s}}\left(r_{\imath}\right) \sigma_{\mathrm{s}}\left(r_{\jmath}\right) h\left(\vec{r}_{\imath}, \vec{r}_{\jmath}\right),
\end{aligned}
$$

where $h$ is the shadowing correlation model, with $-1 \leq h \leq 1$ and $h\left(\vec{r}_{\imath}, \vec{r}_{\jmath}\right)=h\left(\vec{r}_{\jmath}, \vec{r}_{\imath}\right)$. Then, the correlation matrix of $\vec{S}$ conditioned on $\vec{r}_{1}, \ldots, \vec{r}_{N}$ is given by

$$
\mathbf{K}=\left[\begin{array}{cccc}
\sigma_{1}^{2} & \sigma_{1} \sigma_{2} \rho_{1,2} & \cdots & \sigma_{1} \sigma_{N} \rho_{1, N} \\
\sigma_{1} \sigma_{2} \rho_{1,2} & \sigma_{2}^{2} & \cdots & \sigma_{2} \sigma_{N} \rho_{2, N} \\
\vdots & \vdots & \ddots & \vdots \\
\sigma_{1} \sigma_{N} \rho_{1, N} & \sigma_{2} \sigma_{N} \rho_{2, N} & \cdots & \sigma_{N}^{2}
\end{array}\right]
$$

Shadowing correlation models are discussed in Chapter 2, where we conclude with the choice of the following model, which is inspired by [48]:

$$
h\left(r_{\imath}, r_{\jmath}\right)=\max \left\{1-\theta / \theta_{0}, 0\right\} \cdot \max \left\{1-R / R_{0}, 0\right\}
$$

where $\theta$ and $R$ are defined in Section 2.3.3, and the model has two tunable parameters: $0^{\circ}<\theta_{0} \leq 180^{\circ}$ and $R_{0}>0$. Note that the model is separable in $\theta$ and $R$.

We choose this model among many others for the following reasons:

1. In Chapter 2 we show that this model always yields psd correlation matrices $\mathbf{K}$. This is not the case for several of the existing models. 
2. Furthermore, in Chapter 2 we argue that from among all models that always give psd correlation matrices $\mathbf{K}$, this model seemed most physically realistic. In particular we contrast this model with those expressible in the form $h\left(\vec{r}_{\imath}, \vec{r}_{\jmath}\right)=f\left(\left\|\vec{r}_{\imath}-\vec{r}_{\jmath}\right\|\right)$, notably with $f(x)=e^{-x / d_{0}}[43]$. We argue that these models are difficult to reconcile with the propagation arguments for correlation in shadowing given in [48].

3. The selected model has two tunable parameters, and can therefore approximate a wide range of correlation models with reasonable accuracy, as done in [48].

4. The mathematical form of this model lends itself particularly well to fast simulation using shadowing fields, as we demonstrate in Section 5.3.

We choose the values $\theta_{0}=60^{\circ}$ and $R=6 \mathrm{~dB}$, which are typical values based on [48]. This particular model is illustrated in Figure 3.2. Again, this is only one possible model, and our analysis is independent of the form of $h$.

It is primarily the correlation of $\left\{S_{\imath}\right\}$ that represents the analytical challenge and the simulation cost (for high $N$ ), and shadowing correlation is therefore a central focus of our study.

\subsection{Interferer Transmission}

For simplicity, we assume that each IS transmits with equal constant unit power. In Section 6.2.3, we discuss how random transmit power can be incorporated in both analysis and simulation without excessive effort.

\subsection{Total Interference}

The individual interfering signals are added at the $\mathrm{RX}$ to produce a total interference signal. It is often assumed in interference analysis that the total interference power 


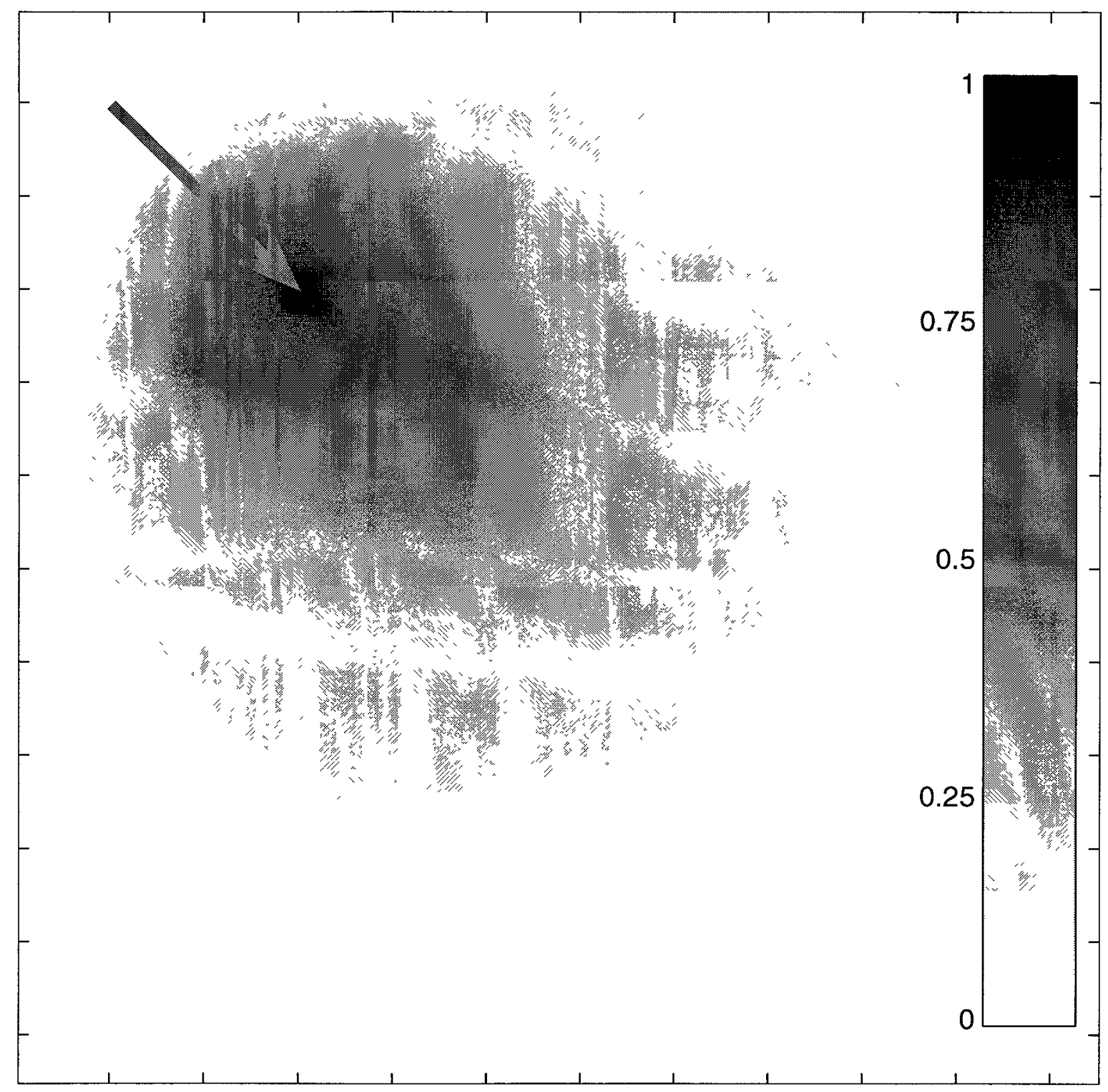

Figure 3.2: Shadowing correlation function (3.5) with $\theta_{0}=60^{\circ}$ and $R_{0}=6 \mathrm{~dB}$ : the correlation coefficient between two shadowed paths beginning at base of the arrow. One path ends at the tip of the arrow, while the other is located anywhere on the plane. The figure is invariant under rotation and scaling. 
is the sum of the individual interference powers, as explained by incoherent signal addition $[42,155-157]$.

We are then interested in finding the statistics of the total interference power given by

$$
I=\sum_{\imath=1}^{N} I_{\imath}, \quad I_{\imath}=p\left(r_{\imath}\right) e^{\lambda S_{\imath}},
$$

where we assume a common constant gain accounting for multiplicative constants such as antenna gains, reference distance, and transmit power, which we set to one without loss of generality. We assume that the RX has an omni-directional receive antenna with normalised unit gain, though it is possible to extend this to a generalised antenna pattern as described in Section 6.2.4. 


\section{Chapter 4}

\section{Analytical Results on the Aggregate Interference}

It would be ideal to find the exact distribution of $I$ in closed form for any choice of channel and system parameters; however, such a solution is unlikely. Firstly,

the complex correlation structure of $\vec{S}$ and its interdependence with $\left\{\vec{r}_{i}\right\}_{i=1}^{N}$ make a closed-form solution unlikely. Secondly, both $g$ and $h$ are almost arbitrary functions of 2 and 4 dimensions respectively, which almost inevitably leads to some involved numerical integration. Nevertheless, there are several things that we can say about $I$ from an analytical point of view.

We first show a simple lognormal approximation for this distribution, which we derive with the aid of a limit theorem that we develop for this purpose. We also study the first two moments of $I$, and observe their asymptotic behaviour. We also propose to reformulate the problem as a stochastic integral. While these results cannot in themselves give us the distribution of $I$, they do help in greatly accelerating the simulation thereof, as described in Section 5.3.

\subsection{Approximate Solution using Exchangeability}

In this section we derive a set of simple equations that approximate, for large $N$, the distribution of $I$ by that of a lognormal. We show in Section 5.2 that this solution, while not solving the problem entirely, provides a good approximation in the case of 
cluster layouts. This solution might also prove useful in the case of a directional RX receive antenna, as suggested in Section 6.2.4.

The method occurs in two steps. First we derive a limit theorem for the sum of exchangeable jointly lognormal RVs. We then map this result onto the physical problem. While this problem is not identical to that of finding the distribution of $I$, there exist enough similarities to justify the mapping, thus providing an approximation that is good under some conditions.

\subsubsection{Limit Theorem on the Sum of Exchangeable Joint Lognormals}

We show that the sum of exchangeable positively correlated jointly lognormal RVs always converges in distribution to a lognormal RV. This stands as a mathematical result in itself in the field of sums of lognormals, but its purpose within this thesis is to formulate a lognormal approximation to the distribution of $I$.

\subsubsection{Problem Formulation}

Let $\left[V_{\imath}\right]_{\imath=1}^{N}$ be a vector of $N$ jointly Gaussian RVs, each with the same mean $\mu$, same

variance $\sigma^{2} \neq 0$, and each pair with the same correlation coefficient $0<\rho \leq 1$. Their correlation matrix can thus be written as

$$
\mathbf{K}_{N \times N}=\sigma^{2}\left(\begin{array}{cccc}
1 & \rho & \cdots & \rho \\
\rho & 1 & \cdots & \rho \\
\vdots & \vdots & \ddots & \vdots \\
\rho & \rho & \cdots & 1
\end{array}\right)
$$

Then $\left[e^{V_{2}}\right]_{\imath=1}^{N}$ is a jointly lognormal vector, characterised by the same parameters. Now let

$$
V=\sum_{\imath=1}^{N} e^{V_{2}}
$$


We say that $V$ follows a sum of exchangeable joint lognormals (SEJLN) distribution with parameters $\mu, \sigma^{2}, \rho, N$. We want to find this distribution, particularly for large $N$.

\subsubsection{Main Result and Proof}

Theorem 12. Let $V$ follow a SEJLN distribution as in (4.2) with parameters $\mu$, $\sigma^{2} \neq 0,0<\rho \leq 1, N \in \mathbb{N}^{*}$. Then, as $N \rightarrow \infty$, the quantity $V / N$ tends in distribution to a lognormal RV with parameters $\left(m_{\infty}, s_{\infty}^{2}\right)$ given by

$$
m_{\infty}=\mu+\frac{1}{2}(1-\rho) \sigma^{2}, \quad s_{\infty}^{2}=\rho \sigma^{2} .
$$

Note that, in the independent case, such as in the CLT, the sum would be normalised by $\sqrt{N}$ because variances would add to each other and the variance of the sum would be $\mathcal{O}(N)$. However, in our correlated case the variance of $V$ is $\mathcal{O}\left(N^{2}\right)$ for $0<\rho \leq 1$, and thus a normalisation by $N$ is appropriate.

In [52] we have given a proof based on a more complicated decomposition. Here, we give a simpler, but essentially similar proof, inspired by the decomposition in [41]: Proof. Let $Z, Z_{1}, \ldots, Z_{N}$ be iid standard Gaussian RVs. Then we can write

$$
V_{\imath}=\mu+\sigma \sqrt{\rho} Z+\sigma \sqrt{1-\rho} Z_{\imath}, \quad \forall i
$$

to obtain the same statistical distribution for $V_{\imath}$ as described in Section 4.1.1.1. This decomposition is along the lines of Theorem 4, where we see that the exhangeble set $\left\{V_{\imath}\right\}$ may be written as a common term $Z$, and individual iid terms $Z_{\imath}$. From (4.2), we have

$$
V / N=e^{\mu+\sigma \sqrt{\rho} Z} \cdot \frac{1}{N} \sum_{\imath=1}^{N} e^{\sigma \sqrt{1-\rho} Z_{\imath}}
$$


Now, from the LLN, we have

$$
\frac{1}{N} \sum_{i=1}^{N} e^{\sigma \sqrt{1-\rho} Z_{\imath}} \stackrel{\mathcal{D}}{\longrightarrow} e^{(1-\rho) \sigma^{2} / 2},
$$

a constant. We may then apply Slutsky's theorem to obtain

$$
V / N \stackrel{\mathcal{D}}{\longrightarrow} e^{\mu+(1-\rho) \sigma^{2} / 2+\sigma \sqrt{\rho} Z},
$$

which is a lognormal RV with parameters given by (4.3).

\subsubsection{Lognormal Approximation for Large $N$}

Given that the SEJLN distribution is lognormal in the limit as $N \rightarrow \infty$, it would be interesting to also examine its behaviour for moderate values of $N$. We reason that since for both $N=1$ and $N \rightarrow \infty$, the SEJLN distribution is lognormal, then it might be approximately lognormal for intermediate values of $N$, and certainly so for large-enough ones. Because a lognormal distribution is uniquely determined by its first and second moments, we can use moment-matching to accurately approximate the SEJLN distribution as long as it remains approximately lognormal. We see in Section 5.2.1 just how good this approximation is. Matching the first two moments of $V$ to those of a lognormal RV, we obtain that $V$ can be approximated in distribution by a lognormal RVs with parameters

$$
\begin{gathered}
m_{V}=\mu+\frac{3}{2} \ln N-\frac{1}{2} \ln \left(1+(N-1) e^{(\rho-1) \sigma^{2}}\right) \\
s_{V}^{2}=\sigma^{2}-\ln N+\ln \left(1+(N-1) e^{(\rho-1) \sigma^{2}}\right) .
\end{gathered}
$$


For high $N$, these expressions simplify to

$$
\begin{aligned}
m_{V} & \approx \mu+\ln N+\frac{1}{2}(1-\rho) \sigma^{2}, \\
s_{V}^{2} & \approx \rho \sigma^{2} \text { for large } N .
\end{aligned}
$$

Since the distribution of $V$ is approximately lognormal for high $N$ per our theorem, it follows that its lognormal parameters are given by (4.9), which is consistent with (4.3), and the additional $\ln N$ term results from the multiplication by $N$.

\subsubsection{Lognormal Approximation to Total Interference Distribution}

Having shown that the SEJLN distribution is asymptotically lognormal, and having obtained expressions for an approximating lognormal distribution for finite $N$, we follow with the second step of the approximate derivation of the distribution of $I$. Indeed, we proceed to map the SEJLN problem onto that of the distribution of $I$.

\subsubsection{Comparing the Two Problems}

Let us make some observations about the individual $e^{V_{\imath}}$ terms, the summands of the SEJLN RV $V$ :

1. They are exchangeable. In fact, the form of correlation matrix (4.1) with $-1 /(N-1) \leq \rho \leq 1$ is the only possible form for an exchangeable set of $N$ RVs [64].

2. They are "augmentable", i.e., adding new terms by increasing $N$ does not change the distribution of the previous terms. This is captured by the fact that $\rho$ does not change with $N$.

3. They are each individually lognormal.

4. They are also jointly lognormal. 
Now, let us look at the same properties for the individual $I_{\imath}$ 's, the summands of I. We have, respectively:

1. As argued before, they are exchangeable.

2. They are also "augmentable". Indeed, adding new interferers should not affect the joint distribution of the previous interferers' powers.

3. They are approximately lognormal, as long as three conditions are met:

(a) The shadowing spread is large enough (say no less than $6 \mathrm{~dB}$ ).

(b) The shadowing spread is not significantly changing over distance, making not only $S_{\imath} \mid r_{\imath}$ but also $S_{\imath}$ approximately Gaussian.

(c) The log-variance of the other terms in $I_{\imath}$ is not too great.

In this case, the lognormal distribution of $e^{\lambda S_{\imath}}$ has an "attractor" property (this is related to the CLT and the Gaussian distribution being "stable".) [14]

4. Given the previous point, it may be vain to consider jointness if the individual $I_{\imath}$ are not even lognormal. However, even the jointness of the terms $S_{\imath}$ is not guaranteed, since their correlation matrix is random (a function of $\vec{r}_{2}$ 's), and therefore we cannot even know if the $I_{\imath}$ 's are approximately jointly lognormal in any sense.

We observe that the two problems are identical in the first two properties, but not in the two others. The last two properties may or may not be fulfilled with any good accuracy, and it is in these two approximations that the inaccuracy of our method lies. We observe in Section 5.2.3 that the approximation is particularly good for a clustered layout. This can be explained as follows: 
1. A clustered layout admits no (or very few) ISs near the RX, which implies that the shadowing spread is (almost) constant for all shadowing paths (see Section $3.2 .2)$.

2. A clustered layout admits only a limited relative spread for $r_{\imath}$, and thus limits the $\log$-variance of $p\left(r_{\imath}\right)$.

3. A clustered layout imposes a quite high expected value of the correlation between any two $S_{2}$ 's, due to their angular proximity. This high correlation seems to affect the jointness of the $S_{\imath}$ 's. This can be intuitively explained thus: the higher the correlation terms, the less "freedom" for the joint distribution of the RVs and thus per force the joint distribution must be close to that of a jointly lognormal set of RVs. Case in point, should it be known that all the correlation coefficients are identically 1 , the joint distribution would admit no freedom at all: all the RVs would be equal and, of course, jointly lognormal.

It would then seem that clustered layouts solve, approximately, the discrepancies between the SEJLN problem and that of the distribution of $I$. As our simulations in Chapter 5 show, our method works well mainly for this kind of layout, probably for the reasons just described, and thus we will in fact make the assumption of a clustered layout in our analysis. This assumption will simplify the channel model somewhat:

1. Since there are few or no ISs near the RX, we may assume the shadowing spread constant (see Section 3.2.2).

2. For the same reason, we may assume the average pathloss as power law with a constant exponent $\beta$ (see Section 3.2.1).

This leads to a simplified expression for the interferers:

$$
I_{\imath}=r_{\imath}^{\beta} e^{\lambda S_{\imath}} .
$$


Having now somewhat restricted the conditions of the interference problem, we may match the problem to the result on SEJLN RVs.

\subsubsection{Cross-Moment-Matching between the Two Problems}

Since we have established that, under the aforementioned conditions, $\left\{I_{\imath}\right\}$ behaves similarly to $\left\{e^{V_{2}}\right\}$, it follows that $I$ behaves similarly to $X$. It would then follow from our limit theorem that $I$ is approximately lognormal for large $N$. We say that this approximating lognormal distribution has parameters $\left(m_{I}, s_{I}^{2}\right)$, which we proceed to derive.

Now, for a given $N$, the SEJLN distribution has three free parameters: $\mu, \sigma$, and $\rho$. It is probably most natural, then, to match the following statistics:

$$
\begin{aligned}
\mathbb{E}\left\{\ln I_{\imath}\right\} & =\mathbb{E}\left\{V_{\imath}\right\}=\mu, \\
\mathbb{E}\left\{\ln ^{2} I_{\imath}\right\} & =\mathbb{E}\left\{V_{\imath}^{2}\right\}=\sigma^{2}+\mu^{2}, \\
\mathbb{E}\left\{\ln I_{\imath} \ln I_{\jmath}\right\} & =\mathbb{E}\left\{V_{\imath} V_{\jmath}\right\}=\rho \sigma^{2}+\mu^{2}, i \neq j .
\end{aligned}
$$

The third expression is in fact a cross-moment of $\left\{\ln I_{2}\right\}$. The relevant statistics of the interference terms can be found from (4.10):

$$
\begin{aligned}
\mathbb{E}\left\{\ln I_{\imath}\right\} & =\mathbb{E}\left\{\lambda S_{\imath}-\beta \ln r_{\imath}\right\}=-\beta G_{1}, \\
\mathbb{E}\left\{\ln ^{2} I_{\imath}\right\} & =\mathbb{E}\left\{\lambda^{2} S_{\imath}^{2}+\beta^{2} \ln ^{2} r_{\imath}-2 \beta \lambda S_{\imath} \ln r_{\imath}\right\}=\lambda^{2} \sigma_{\mathrm{s}}^{2}+\beta^{2} G_{2}, \\
\mathbb{E}\left\{\ln I_{\imath} \ln I_{\jmath}\right\} & =\mathbb{E}\left\{\lambda^{2} S_{\imath} S_{\jmath}+\beta^{2} \ln r_{\imath} \ln r_{\jmath}\right\}-\beta \lambda \mathbb{E}\left\{S_{\jmath} \ln r_{\imath}+S_{\imath} \ln r_{\jmath}\right\} \\
& =\lambda^{2} \sigma_{\mathrm{s}}^{2} G_{\mathrm{cor}}+\beta^{2} G_{1}^{2}, i \neq j,
\end{aligned}
$$

where

$$
\begin{aligned}
G_{n} & =\mathbb{E}\left\{\ln ^{n} r_{\imath}\right\}, \\
G_{\text {cor }} & =\frac{\mathbb{E}\left\{S_{\imath} S_{\jmath}\right\}}{\sigma_{\mathrm{s}}^{2}}, i \neq j,
\end{aligned}
$$


terms that need to be evaluated numerically in general.

Equating (4.11) with (4.12) gives:

$$
\begin{aligned}
\mu & =-\beta G_{1}, \\
\sigma^{2} & =\beta^{2}\left(G_{2}-G_{1}^{2}\right)+\lambda^{2} \sigma_{\mathrm{s}}^{2}, \\
\rho & =\frac{\lambda^{2} \sigma_{\mathrm{s}}^{2}}{\sigma^{2}} G_{\mathrm{cor}} .
\end{aligned}
$$

We substitute these into (4.8) and, by equating $\left(m_{I}, s_{I}^{2}\right)=\left(m_{V}, s_{V}^{2}\right)$, we obtain the solution.

\subsubsection{Simple Semi-Analytical Solution}

From the above derivation, we find that $I$ may be approximated by a lognormal RV with parameters

$$
\begin{aligned}
m_{I} & =\frac{3}{2} \ln N-\beta G_{1}-\frac{1}{2} \ln \left(1+(N-1) e^{\left(\lambda^{2} \sigma_{\mathrm{s}}^{2}\left(G_{\mathrm{cor}}-1\right)-\beta^{2}\left(G_{2}-G_{1}^{2}\right)\right)}\right), \\
s_{I}^{2} & =\lambda^{2} \sigma_{\mathrm{s}}^{2}-\ln N+\beta^{2}\left(G_{2}-G_{1}^{2}\right)+\ln \left(1+(N-1) e^{\left(\lambda^{2} \sigma_{\mathrm{s}}^{2}\left(G_{\mathrm{cor}}-1\right)-\beta^{2}\left(G_{2}-G_{1}^{2}\right)\right)}\right) .
\end{aligned}
$$

Since we are interested in large $N$, and because the SEJLN distribution may not be close to lognormal for smaller $N$, we also formulate a simpler expression for large $N$ :

$$
\begin{aligned}
m_{I} & \approx \ln N+\frac{1}{2} \beta^{2}\left(G_{2}-G_{1}^{2}\right)-\beta G_{1}+\frac{1}{2} \lambda^{2} \sigma_{\mathrm{s}}^{2}\left(1-G_{\mathrm{cor}}\right), \\
s_{I}^{2} & \approx \lambda^{2} \sigma_{\mathrm{s}}^{2} G_{\mathrm{cor}} .
\end{aligned}
$$

$G_{n}$ and $G_{\text {cor }}$ are to be found by numerical integration of the functions $g$ and $h$ :

$$
\begin{aligned}
G_{n} & =\iint_{\mathcal{A}_{g}}(\ln r)^{n} g(\vec{r}) d \vec{r} \\
G_{\mathrm{cor}} & =\iint_{\mathcal{A}_{g}} \iint_{\mathcal{A}_{g}} h\left(\vec{r}_{1}, \vec{r}_{2}\right) g\left(\vec{r}_{1}\right) g\left(\vec{r}_{2}\right) d \vec{r}_{1} d \vec{r}_{2} .
\end{aligned}
$$


These integrals are well-behaved and can be well approximated by a Riemann sum (trapezoidal rule) with a moderate number of terms. $G_{n}$ and $G_{\text {cor }}$ are termed "geometric coefficients", and are an idea borrowed from $[158,159]$. It should be noted that this numerical integration is inevitable, due to the limitless possibilities for the expressions of the functions $g$ and $h$. However, our solution is elegant in that it separates these integrals from the scalar quantities $\beta, \sigma_{\mathrm{s}}$, and $N$, in which it is purely analytical. For this reason, and the brevity of the final equations, we believe this solution to be the simplest possible while not being simplistic.

Another important point to observe in our solution is that only one scalar quantity depends on the shadowing correlation function $h$, namely $G_{\text {cor }}$. This implies a large loss of information about the channel model, which leads us to formulate a guideline: while it is very important to include correlation in our model, only $G_{\text {cor }}=\mathbb{E}\left\{h\left(\vec{r}_{1}, \vec{r}_{2}\right)\right\}$ (where $\vec{r}_{1}, \vec{r}_{2}$ are iid according to $g(\vec{r})$ ) is relevant to the analysis, which is much easier to measure and estimate than all of $h$. In general, we think this kind of analysis can point the way for engineers doing channel measurements to know what statistics are actually relevant to particular problems, thus greatly reducing measurement efforts.

We may interpret this result through Theorem 4: indeed, it would seem that each shadowing term is approximately composed of a lognormal component with spread $\sqrt{G_{\text {cor }}} \sigma_{\mathrm{s}}$ common to every path, as well as an additional lognormal term that is iid for every path. As the paths add, the common term remains, while the iid terms vanish as a consequence of the LLN, which is similar to our proof of the convergence of the SEJLN distribution. This interpretation is also coherent with the physical model presented in Figure 2.5. 


\subsection{Study of Moments}

We now study the first two moments of the total interference $I$. This approach is interesting for three reasons:

1. The moments (of positive integer order) are probably the easiest statistics to derive in such problems.

2. The study of interference problems has historically started with moment analysis [13], which has yielded good initial information.

3. The study of these moments gives us valuable insight into the asymptotic (as $N \rightarrow \infty$ ) behaviour of $I$, which shows the importance of modeling correlation in shadowing, and also leads to some useful equations that can simplify lengthy simulations, as seen in Section 5.3.6.

\subsubsection{Formulation of Moments}

We begin by evaluating the following quantities:

$$
\begin{aligned}
& A=\mathbb{E}\left\{I_{1}\right\}=\mathbb{E}\left\{p\left(r_{1}\right) e^{\lambda S_{1}}\right\}, \\
& B=\mathbb{E}\left\{I_{1}^{2}\right\}=\mathbb{E}\left\{p^{2}\left(r_{1}\right) e^{2 \lambda S_{1}}\right\}, \\
& C=\mathbb{E}\left\{I_{1} I_{2}\right\}=\mathbb{E}\left\{p\left(r_{1}\right) p\left(r_{2}\right) e^{\lambda\left(S_{1}+S_{2}\right)}\right\} .
\end{aligned}
$$

Now, while the set $\left\{S_{\imath} \mid \vec{r}_{\imath}\right\}$ is not exchangeable, since the RVs may have different variances, and pairs may have different correlation coefficients, the set $\left\{S_{\imath}\right\}$ is exchangeable, and so is $\left\{I_{\imath}\right\}$. From this, we may easily formulate the moments:

$$
\begin{aligned}
\mathbb{E}\{I\} & =N A, \\
\mathbb{E}\left\{I^{2}\right\} & =N B+\left(N^{2}-N\right) C, \\
\mathbb{V A R}\{I\} & =N(B-C)+N^{2}\left(C-A^{2}\right) .
\end{aligned}
$$


We observe that, while for independent shadowing we have $C=A^{2} \Rightarrow \operatorname{VAR}\{I\}=$ $\mathcal{O}(N)$, in general for correlated shadowing $\mathbb{V A R}\{I\}=\mathcal{O}\left(N^{2}\right)$. The mean power of $I$ remains the same regardless of correlation. Therefore what was already observed for small $N$ [155] will be even more significant for large $N$ : adding correlation changes (specifically, broadens) the distribution of $I$ significantly. It follows that, given a sufficiently realistic shadowing correlation model, the distribution of $I$ obtained using correlated shadowing will be much more realistic than that obtained using independent shadowing.

Also, because of the asymptotic behaviour of the mean and variance, analysing $I$ as $N \rightarrow \infty$ requires the study of the convergence of $I / N$ (rather than $(I-\mathbb{E}\{I\}) / \sqrt{N}$ in the independent case). Because of the existence of correlation, the CLT cannot be applied for large $N$. Indeed $I / N$ does not necessarily converge to a Gaussian distribution, and may in fact converge to a distribution close to a lognormal with a significant spread, as we see in Section 5.2.3.

We can therefore conclude that correlation in shadowing becomes a dominating factor in the distribution of $I$ as $N$ becomes large.

\subsubsection{Numerical Evaluation of Moments}

While we may estimate the expectations of (4.18) via Monte Carlo simulation, we find it faster and more exact to evaluate them through numerical integration. By first conditioning on $r_{1}$ we have:

$$
A=\int_{r_{\min }}^{r_{\max }} \bar{g}(r) p(r) e^{\frac{1}{2} \lambda^{2} \sigma_{\mathrm{s}}^{2}(r)} d r
$$

where $\bar{g}(r)$ is the pdf of $r_{\imath}$, evaluated from $g(\vec{r})$ as follows:

$$
\bar{g}(r)=r \int_{0}^{2 \pi} g(\vec{r}) d \angle \vec{r}
$$


Similarly,

$$
B=\int_{r_{\min }}^{r_{\max }} \bar{g}(r) p^{2}(r) e^{2 \lambda^{2} \sigma_{\mathbf{s}}^{2}(r)} d r
$$

Finally,

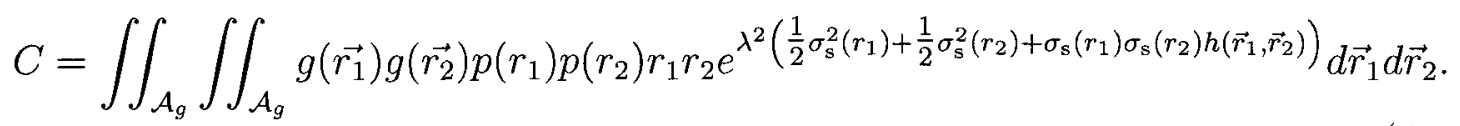

This integral needs to be evaluated in four dimensions for a general correlation model $h$, and is not easy to separate in the case of model (3.5).

\subsubsection{Using Moments in the Large $-N$ Regime}

Having described a fairly straightforward method for obtaining the first two moments of $I$, we may use this information to assist in finding the approximate distribution of $I$ when $N$ is large. To do this, consider first the limiting distribution of $I / N$ as $N \rightarrow \infty$. While we do not know the shape of this distribution, we may conjecture that such a limiting distribution exists, since $I$ is the sum of exchangeable RVs [64]. It is not necessary to know anything about the shape of this limiting distribution to see that, once $N$ is large enough, one would expect the shape of the cdf of $I$ to stabilise, and the cdf merely shift according to $N$. This leads to the following two related ideas:

1. If we know the distribution of $I$ for some large $N$ (e.g., through simulation), we may extrapolate its behaviour for some larger number of interferers $N M$ $(M>1)$, using knowledge of moments to shift the cdf appropriately while maintaining its shape.

2. Conversely, if we obtain the limiting distribution of $I / N$ as $N \rightarrow \infty$ (which we at this point do not know how to do), the distribution of $I$ for some large $N$ can likewise be approximated by shifting the limiting distribution appropriately 
using the knowledge of moments.

In both cases, we derive equations that apply some affine (linear plus constant) transformation to a known cdf in order to obtain the cdf of $I$ for some different large $N$. The linear transformation preserves the shape in the linear and $\mathrm{dB}$ domains, but the added constant will deform the shape in the case of the $\mathrm{dB}$ domain only. To achieve these transformations, three methods present themselves: using the mean only, using the variance only, and using both. We will explore this approach in detail in Section 5.3.6.

\subsubsection{Mean-Matching}

The case of mean-matching is the simplest, as the mean of $I$ is simply proportional to $N$, and we need not compute the moments of $I$ explicitly. We then have

$$
\sum_{i=1}^{N M} I_{\imath} \stackrel{\mathcal{D}}{\approx} M \sum_{\imath=1}^{N} I_{\imath}, \quad M \geq 1, \quad N \text { large }
$$

and

$$
\sum_{\imath=1}^{N} I_{\imath} \stackrel{\mathcal{D}}{\approx} N\left(\lim _{N^{\prime} \rightarrow \infty} \frac{1}{N^{\prime}} \sum_{\jmath=1}^{N^{\prime}} I_{\jmath}\right), \quad N \text { large }
$$

two simple expressions to shift the cdf of $I$ within the large- $N$ regime.

\subsubsection{Variance-Matching}

A different linear transformation may be obtained by matching not the mean, but only the variance between the available and desired distributions. In this case we also have a finite and asymptotic version.

In the finite case, we have

$$
\sum_{\imath=1}^{N M} I_{\imath} \stackrel{\mathcal{D}}{\approx} c \sum_{\imath=1}^{N} I_{\imath}, \quad M \geq 1, \quad N \text { large }
$$


where

$$
c=\sqrt{\frac{N M(B-C)+N^{2} M^{2}\left(C-A^{2}\right)}{N(B-C)+N^{2}\left(C-A^{2}\right)}} .
$$

Now, in the asymptotic case, we observe that

$$
\mathbb{V A R}\{I / N\} \longrightarrow C-A^{2}, \quad \text { as } N \rightarrow \infty
$$

It follows that

$$
\sum_{\imath=1}^{N} I_{\imath} \stackrel{\mathcal{D}}{\approx} c^{\prime}\left(\lim _{N^{\prime} \rightarrow \infty} \frac{1}{N^{\prime}} \sum_{\jmath=1}^{N^{\prime}} I_{\jmath}\right), \quad N \text { large }
$$

where

$$
c^{\prime}=\sqrt{N^{2}+N \frac{B-C}{C-A^{2}}} .
$$

Variance--matching is not very different from mean-matching, in that they both multiply the data by a constant factor, and $c \approx M$ and $c^{\prime} \approx N$ for high $N$.

\subsubsection{Matching both Mean and Variance}

Since the first two moments of $I$ are always easily obtainable from (4.19), the scaling approach can be refined using both moments in the following respective ways:

$$
\sum_{\imath=1}^{N M} I_{\imath} \stackrel{\mathcal{D}}{\approx} b+a \sum_{\imath=1}^{N} I_{\imath}, \quad M \geq 1, \quad N \text { large }
$$

where

$$
\begin{aligned}
& a=\sqrt{\frac{M\left(B-C+N M\left(C-A^{2}\right)\right)}{B-C+N\left(C-A^{2}\right)}}, \\
& b=N A(M-a) .
\end{aligned}
$$


Similarly, beginning with

$$
\begin{aligned}
\mathbb{E}\{I / N\} & \longrightarrow A, \\
\mathbb{E}\left\{(I / N)^{2}\right\} & \longrightarrow C, \\
\operatorname{VAR}\{I / N\} & \longrightarrow C-A^{2}, \quad \text { as } N \rightarrow \infty,
\end{aligned}
$$

we obtain

$$
\sum_{\imath=1}^{N} I_{\imath} \stackrel{\mathcal{D}}{\approx} b^{\prime}+a^{\prime}\left(\lim _{N^{\prime} \rightarrow \infty} \frac{1}{N^{\prime}} \sum_{\jmath=1}^{N^{\prime}} I_{\jmath}\right), \quad N \text { large }
$$

where

$$
\begin{aligned}
& a^{\prime}=c^{\prime} \text { in }(4.30), \\
& b^{\prime}=A\left(N-a^{\prime}\right) .
\end{aligned}
$$

Again, this matching is not very different from the previous approaches, since for large $N$ we have $a^{\prime}$ approaching $N$ and $b^{\prime}$ approaching zero. An important difference is that adding the $b^{\prime}$ term does not allow the approximating distribution to take values in the neighbourhood of zero, which may cause high relative error in the lower tail.

\subsection{Problem Reformulation Using a Stochastic Integral}

It would be interesting to actually find the limiting distribution of $I / N$. While we do not have a solution to this problem, we offer here a reformulation of the problem from an infinite sum into a stochastic integral that does not depend on $N$, which might be a first step towards the analysis of this problem.

This reformulation also motivates an alternative simulation algorithm, namely shadowing fields, which proves computationally efficient for high $N$, and is described in Section 5.3.1. 


\subsubsection{Equivalent Stochastic Integral for $I / N$ as $N \rightarrow \infty$}

Consider a two-dimensional Gaussian shadowing field $S(\vec{r}), \vec{r} \in \mathbb{R}^{2} \backslash 0$, such that if we assign $S_{\imath}=S\left(\vec{r}_{\imath}\right)$, then the $S_{\imath}$ 's have the desired joint distribution. Then $S(\vec{r})$ is a stochastic field. Now, as we observe that since the values $S_{\imath}$ essentially sample this random field, the expression for $I$ is actually a stochastic numerical integration of a function of $S(\vec{r})$. The corresponding integral would take the following form:

$$
\frac{I}{N} \stackrel{\mathcal{D}}{\longrightarrow} \int_{\mathbb{R}^{2}} g(\vec{r}) p(\|\vec{r}\|) e^{\lambda S(\vec{r})} d \vec{r} \text { as } N \rightarrow \infty
$$

\subsubsection{Mathematical Argument}

The following is not an exact proof, as the convergence of various quantities is not proved but assumed. The argument assumes that $S(\vec{r})$ is correlated enough so that small variations in $\vec{r}$ give correspondingly small variations in $S(\vec{r})$ : this is probably the case for the model (3.5), but not if shadowing is, e.g., independent.

Consider a square region $\mathcal{A}$ of side length $\delta$ such that $\mathcal{A}_{g} \subset \mathcal{A}$, and divide $\mathcal{A}$ into $L \times L$ uniform square regions $\mathcal{A}_{l}, l=1, \ldots, L^{2}$. Also, let $\vec{q}$ be the centre of square $\mathcal{A}_{l}$. Let us fix $N=L^{3}$ and let $L \rightarrow \infty$.

We have

$$
\frac{I}{N}=\frac{1}{N} \sum_{\imath=1}^{N} p\left(r_{\imath}\right) e^{\lambda S_{\imath}}=L^{-3} \sum_{l=1}^{L^{2}} \sum_{\jmath=1}^{J_{l}} p\left(r_{l, j}\right) e^{\lambda S_{l, 3}},
$$

where the indices have been remapped as $i \mapsto l, j$, such that for every $l, \vec{r}_{l, j} \in \mathcal{A}_{l}$.

Since $N \rightarrow \infty \Rightarrow L \rightarrow \infty \Rightarrow\left\|\vec{r}_{l, j}-\vec{q}_{l}\right\|<\delta / \sqrt{2} L \rightarrow 0$, we can now make the following approximations:

$$
p\left(r_{l, J}\right) \approx p\left(q_{l}\right)
$$

where $q_{l}=\left\|\vec{q}_{l}\right\|$, and assuming $p(r)$ does not vary too quickly over a small distance. 
Also,

$$
S_{l, \jmath}=S\left(\vec{r}_{l, \jmath}\right) \approx S\left(\vec{q}_{l}\right)=S_{l}
$$

assuming that $\sigma_{\mathrm{s}}(r)$ does not change too quickly over a small distance, and from the fact that the values of $S(\vec{r})$ at two nearby points have a correlation coefficient close to 1 . We then have $S(\vec{r})$ almost constant over $\mathcal{A}_{l}$ with high probability.

These two approximations lead to:

$$
\frac{I}{N} \approx L^{-3} \sum_{l=1}^{L^{2}} p\left(q_{l}\right) e^{\lambda S_{l}} J_{l}
$$

Now $J_{l}$ is a binomial RV with parameters $L^{3}$ and $\int_{\mathcal{A}_{l}} g(\vec{r}) d \vec{r} \approx g\left(\vec{q}_{l}\right) \delta^{2} / L^{2}$, and therefore

$$
\begin{aligned}
\mathbb{E}\left\{J_{l} / L\right\} & =g\left(\vec{q}_{l}\right) \delta^{2}, \\
\operatorname{VAR}\left\{J_{l} / L\right\} & =L^{-1} g\left(\vec{q}_{l}\right) \delta^{2}\left(1-g\left(\vec{q}_{l}\right) \delta^{2} / L^{2}\right) \approx L^{-1} g\left(\vec{q}_{l}\right) \delta^{2} .
\end{aligned}
$$

We observe that the variance of $J_{l} / L$ is $\mathcal{O}\left(L^{-1}\right)$ and hence vanishes. It follows that the quantity converges in distribution to its mean (this is a similar argument to the one used in the proof of the LLN [61, p.275]), and we may write:

$$
\frac{I}{N} \approx(\delta / L)^{2} \sum_{l=1}^{L^{2}} p\left(q_{l}\right) e^{\lambda S_{l}} g\left(\vec{q}_{l}\right) .
$$

This last expression, as $L \rightarrow \infty$, is essentially a Riemann integral expression of (4.36).

\subsection{Summary}

Various analytical tools can help with the derivation of the approximate distribution of $I$. We follow with a chapter on simulation, where we show how these approximate solutions perform against Monte Carlo simulations, and also show how some analytical results may assist in the accelerated simulation of said distribution. 


\section{Chapter 5}

\section{Simulation Results and Optimisation}

Simulation carries significant weight in our work. On the one hand, we use Monte Carlo simulation to verify the accuracy of the approximate solution for the distribution of $I$ developed in Section 4.1. On the other hand, our analytical solution proves accurate only in certain cases, and we may need to resort to a simulation approach in general. To this end, we find that we can greatly simplify the Monte Carlo sim-

ulations via various analytical results from Sections 4.2 and 4.3 , thus resulting in a mixed simulation-numerical-analytical method.

These two approaches really serve to solve the same problem: how to find the distribution of $I$ as quickly as possible and with good accuracy, by any means necessary.

\subsection{Simulation Setup}

We begin by describing the basic simulator used to obtain the cdf of $I$ via Monte Carlo simulation.

\subsubsection{Simulation Platform}

We now describe the software and hardware parameters used to perform the simulations in this thesis. These are of particular interest when we examine the time 
performance of various simulation algorithms in Section 5.3. However, we do not expect the relative time gains between different simulation setups to vary much, for two reasons:

1. The relative simulation times tend to roughly correspond to what would be expected theoretically.

2. We spent a significant amount of time optimising the simulation code, so that the majority of computational time is devoted to mathematical operations and not software overhead.

The hardware and software specifications of the simulation platform are as follows:

- Intel Core $^{\mathrm{TM}_{\mathrm{i}}} 860,2.8 \mathrm{GHz}, 8$ logical cores (4 physical cores with hyperthreading).

- 8 GB RAM.

- Microsoft@ Windows® 7 Professional, 64 bit.

- The MathWorks ${ }^{\text {TM }}$ MATLABß version 7.9.0.529 (R2009b).

We observed that MATLAB automatically uses several of the logical cores in parallel to execute large repetitive tasks. The repetitive nature of the algorithms lends itself particularly well to hardware parallelisation.

\subsubsection{Traditional Approach: Matrix Factorisation}

The principle challenge in the Monte Carlo simulation of $I$ is the joint simulation of $\vec{S}$ given $\left\{\vec{r}_{\imath}\right\}_{2=1}^{N}$. Generating a Gaussian vector of arbitrary correlation matrix $\mathbf{K}$ is often $[48,57,102,113,160,161]$ done by solving for $\mathbf{C}_{N \times N}$ in the equation

$$
\mathbf{K}=\mathbf{C}^{T} \mathbf{C},
$$


for each particular realisation of $\mathbf{K}$. We write $\mathbf{C}=\sqrt[*]{\mathbf{K}}$. The next step is to generate a vector $\vec{Z}=\left[Z_{i}\right]_{i=1}^{N}$ of independent standard Gaussian $\mathcal{N}(0,1)$ RVs. $\vec{S}$ is then obtained from

$$
\vec{S}=\vec{Z} \mathbf{C}
$$

This is implemented as follows:

\section{Algorithm using Matrix FACtorisation}

Ensure: The histogram of $I[k]$ approximates the pdf of $I$.

$$
\text { for } \begin{aligned}
k & =1 \text { to } K \text { do } \\
\text { for } i & =1 \text { to } N \text { do } \\
\vec{r}_{\imath} & \Leftarrow i . i . d . \text { random from } g(\vec{r}) \\
Z_{\imath} & \Leftarrow \text { i.i.d. random } \mathcal{N}(0,1)
\end{aligned}
$$

end for

for $i=1$ to $N$ do

$$
\begin{aligned}
& \bar{K}[i, i] \Leftarrow 1 \\
& \text { for } j=1 \text { to } i-1 \text { do } \\
& \qquad \bar{K}[i, j]=\bar{K}[j, i] \Leftarrow h\left(\vec{r}_{\imath}, \vec{r}_{\jmath}\right)
\end{aligned}
$$

\section{end for}

end for

$\overline{\mathbf{C}} \Leftarrow \sqrt[*]{\overline{\mathbf{K}}}$

$\vec{S} \Leftarrow \operatorname{diag}\left(\sigma_{\mathrm{s}}\left(\vec{r}_{1}\right), \ldots, \sigma_{\mathrm{s}}\left(\vec{r}_{N}\right)\right) \cdot \vec{Z} \cdot \overrightarrow{\mathbf{C}}$

$I[k] \Leftarrow \sum_{\imath=1}^{N} p\left(r_{\imath}\right) e^{\lambda S_{\imath}}$

\section{end for}

There exist various algorithms for calculating $\mathbf{C}$. In the case when $\mathbf{K}$ is positive definite, Cholesky factorisation is a stable algorithm $[113,160]$ that gives a triangular 
solution for $\mathbf{C}$. However, $\mathbf{K}$ is allowed to be psd in general ${ }^{1}$. Also, numerical rounding can make $\mathbf{K}$ slightly non-psd. To fix this double problem there are at least two procedures. One is to modify $\mathbf{K}$ slightly so that it becomes positive definite [113]. However, $\mathbf{K}$ need not be positive definite (as suggested in $[57,102,113]$ ), but may be psd in general. In this case, Cholesky factorisation is not applicable, and matrix diagonalisation (eigenvalue decomposition) $[66,70,114,142]$ should be used. Also, should some of the resulting eigenvalues of $\mathbf{K}$ be slightly negative due to rounding, they can be set to zero [114]. Then, if there are eigenvalues equal to zero, $\mathbf{K}$ is not full rank and therefore not positive definite, but $\mathbf{C}$ can still be found. Additionally, if $\mathbf{K}$ is highly-correlated, a fast approximation exists [162].

While Cholesky factorisation fails for singular matrices $\mathbf{K}$, we have observed in simulations that this event is extremely rare using model (3.5) with double-precision arithmetic. Therefore, we simply use Cholesky factorisation, and reject the extremely rare simulations where this method fails.

\subsubsection{Representing the Simulation Results}

To obtain an accurate estimate of the cdf of $I$, we generate $K=1000000$ trials of $I$, and plot their empirical cdfs. This tends to give smooth curves at least in the probability interval $\left[10^{-4}, 1-10^{-4}\right]$. The cdf is plotted on "lognormal paper" [157], on which the axes are deformed in such a way that all (and only) lognormal distributions appear as straight lines with positive slope. This is becoming common practice in interference work [22,23], and is useful in our case, since we propose a lognormal approximation to the distribution of $I$; thus, we can evaluate whether a lognormal approximation is possible by looking at the linearity of the estimated cdf on lognormal paper.

\footnotetext{
${ }^{1}$ The case when $\mathbf{K}$ is psd but not positive definite corresponds to a singular matrix. In practice, for randomly generated positions $\vec{r}_{\imath}$ and using double-precision arithmetic, we find this event to be extremely rare in our simulations, and thus Cholesky factorisation is almost safe.
} 


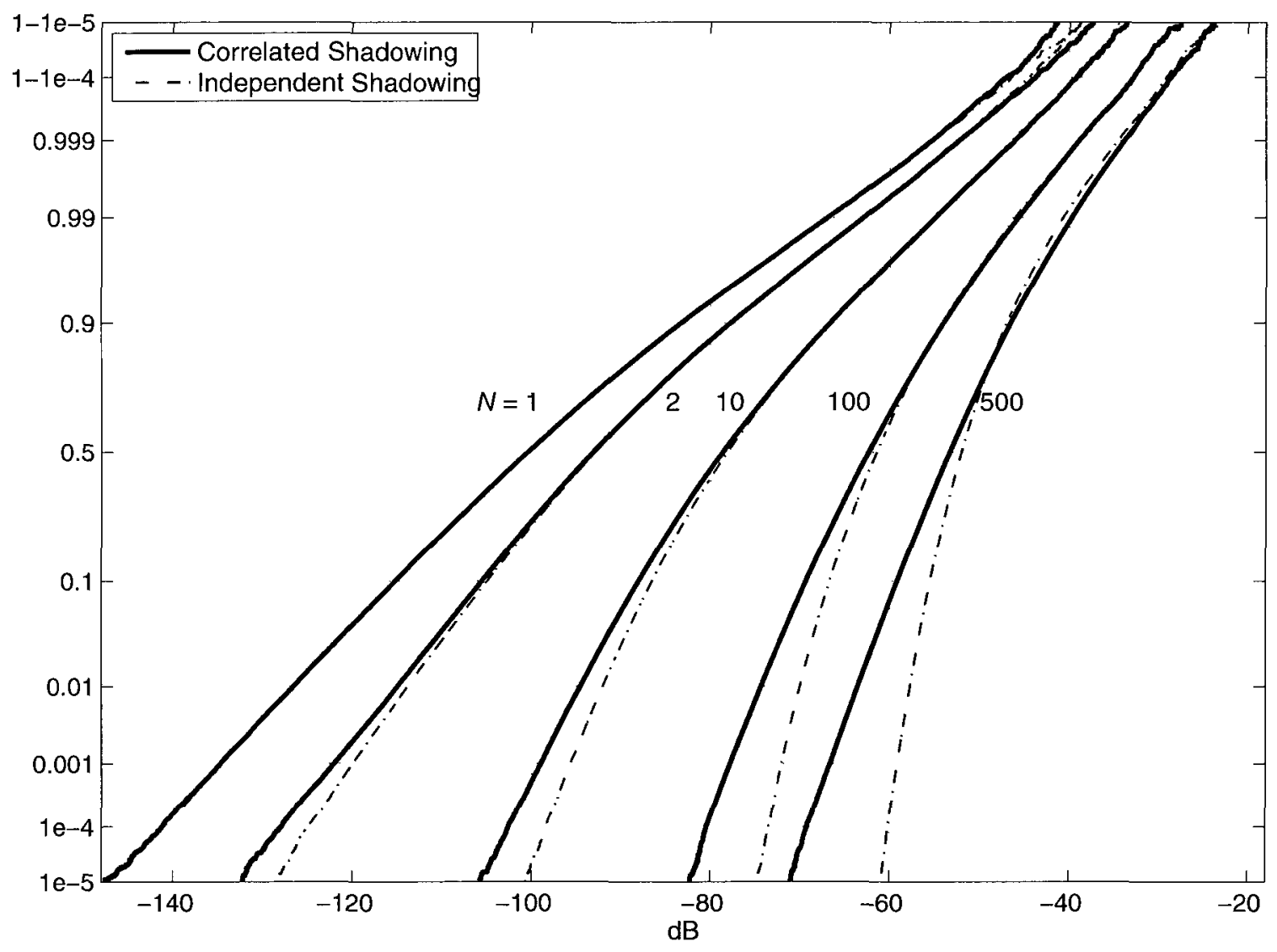

Figure 5.1: Interference cdfs on lognormal paper: with and without shadowing correlation. 


\subsubsection{Correlated versus Independent Shadowing}

As a first exercise, let us observe the interference cdfs as obtained from the described simulation approach. We compare these empirical cdfs to those obtained from assuming only independent shadowing, but with the same marginal distribution. Figure 5.1 illustrates this comparison for varying $N$. While for $N=1$ the obtained cdfs are essentially the same (theoretically, they are identical, since there is no correlation among just one IS), and while for small $N$ correlation does not change the cdf significantly, for higher $N$ the gap increases between the cdf using independent versus correlated shadowing. Specifically, we observe a broadening of the cdf when correlation is included, with respect to the cdf obtained using independent shadowing, an effect which increases with $N$. This is expected from the rate of growth of the variance of $I$, as predicted from (4.19). This supports our claim that shadowing correlation should not be neglected when studying the interference from large networks.

\subsection{Evaluating the Accuracy of the Lognormal Approxima- tion Method}

In Section 4.1.2 we developed a simple semi-analytical approximation to the distribution of $I$. The argument is predicated on the convergence of the SEJLN distribution to a lognormal. We first test the accuracy and rate of convergence of this claim, after which we evaluate how well, and in what cases, our semi-analytical solution works.

\subsubsection{Accuracy of Convergence of the SEJLN Distribution to the Log- normal}

Equations (4.8) and (4.9) both give, with a different degree of accuracy, the parameters of a lognormal approximation to the SEJLN distribution. We wish to evaluate the validity of these approximations as a function of the SEJLN parameters. Since 
$e^{\mu}$ is a scaling factor, we may set $\mu=0$ without loss of generality. We then fix the parameters $\rho$ and $\sigma$ and increase $N$. We show simulations for $\sigma=6 \mathrm{~dB}$ and $12 \mathrm{~dB}$, and for $\rho=0.5,0.05$, and 0.005 . Note that for the equations to apply, the value of $\sigma$ must be converted from $\mathrm{dB}$ to natural units as follows: $\sigma[$ nat $]=\lambda \times \sigma[\mathrm{dB}]$. Therefore, $\sigma \cong 1.382$ and 2.763 .

In Figures 5.2 through 5.7, we show Monte Carlo simulations of the SEJLN distribution computed using (4.4), which is an exact algorithm for simulating the SEJLN distribution in $\mathcal{O}(N)$ time. We compare the simulations to the lognormals with parameters (4.8) and (4.9). We plot the distributions on lognormal paper to evaluate the convergence to the lognormal distribution.

We can make the following observations from these figures:

1. As per our theorem, for all $0<\rho \leq 1$ and $\sigma^{2} \neq 0$, the SEJLN distribution tends toward a lognormal distribution with known parameters as $N \rightarrow \infty$.

2. The SEJLN distribution is (trivially) lognormal for $N=1$. For $N \geq 2$, the SEJLN distribution becomes less and less lognormal until a certain $N$, and then begins to converge back to a lognormal distribution. Once it is again approximately lognormal for a certain $N$, it remains approximately lognormal for any larger $N$.

3. The convergence of the SEJLN to a lognormal is faster for higher $\rho$ and for lower $\sigma$.

4. Though the SEJLN distribution converges to a lognormal, this convergence is not uniform over the whole range of values and in fact never converges at arbitrarily large values, where the behaviour is instead known to be that of the heaviest term in the sum times the number of these heaviest terms, for $-1 \leq \rho<1$ (as long as the matrix remains psd) [17]. This is notably visible in 


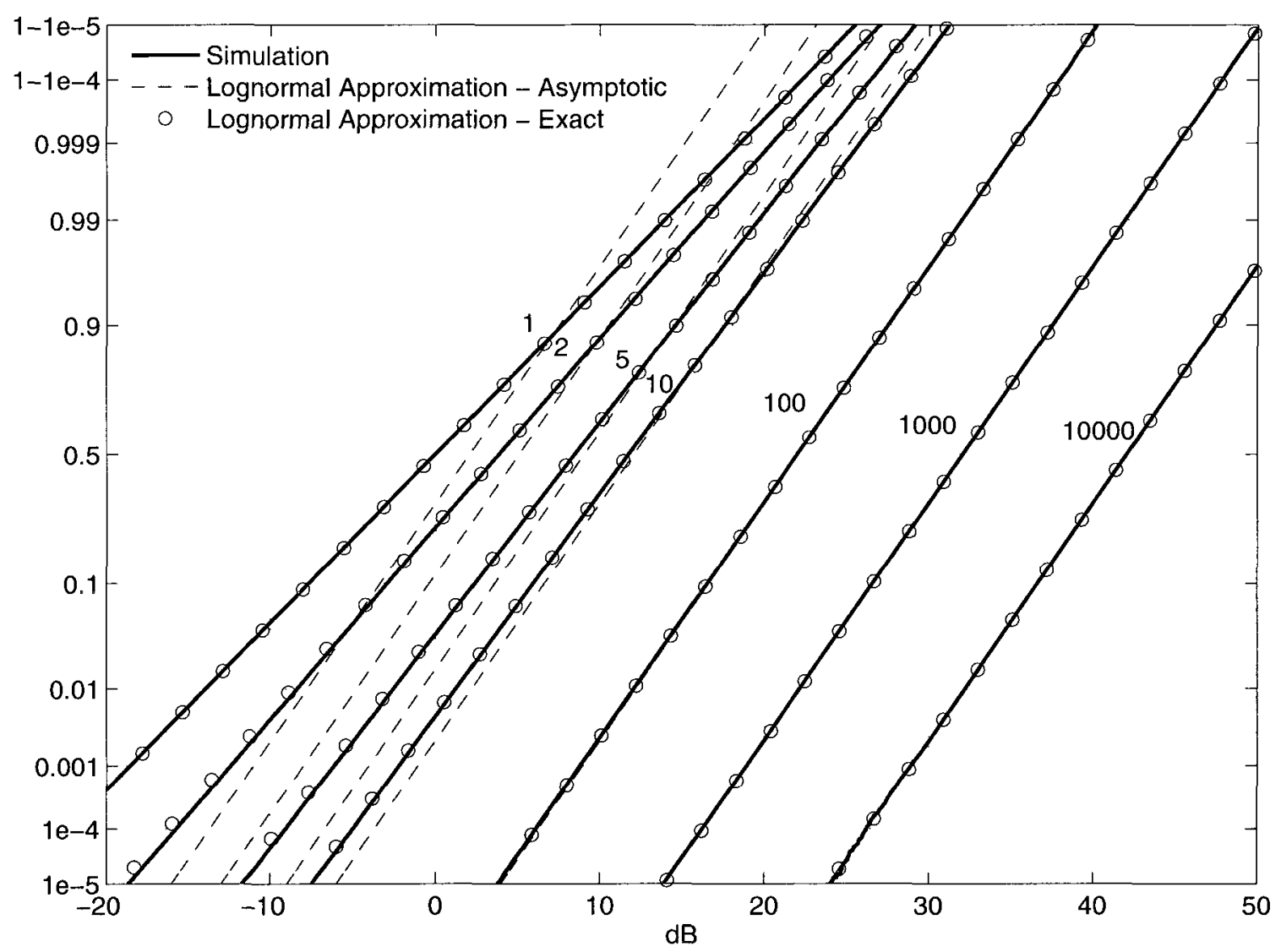

Figure 5.2: SEJLN cdf on lognormal paper, $\sigma=6 \mathrm{~dB}, \rho=0.5$, with varying $N$. 


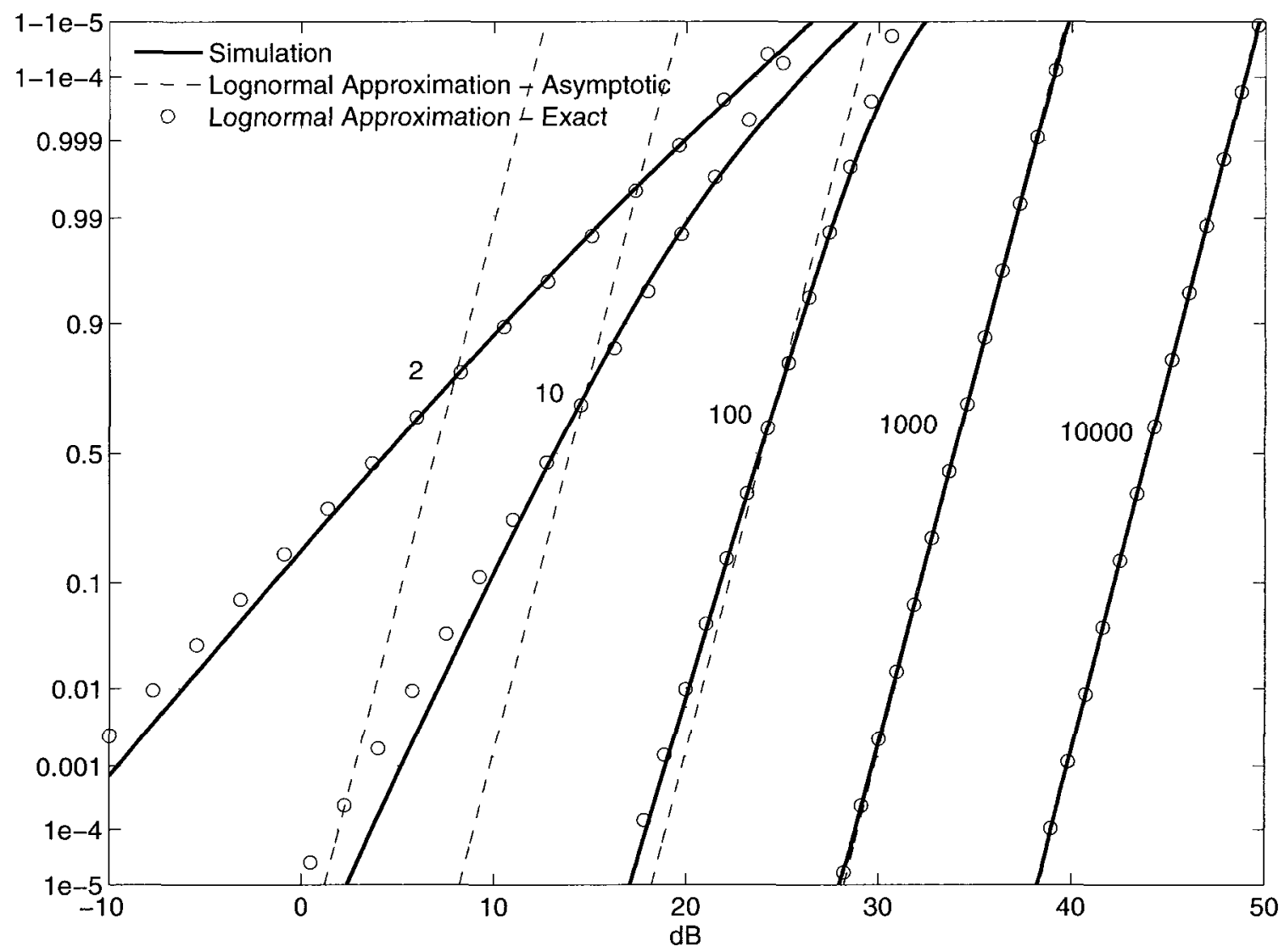

Figure 5.3: SEJLN cdf on lognormal paper, $\sigma=6 \mathrm{~dB}, \rho=0.05$, with varying $N$. 


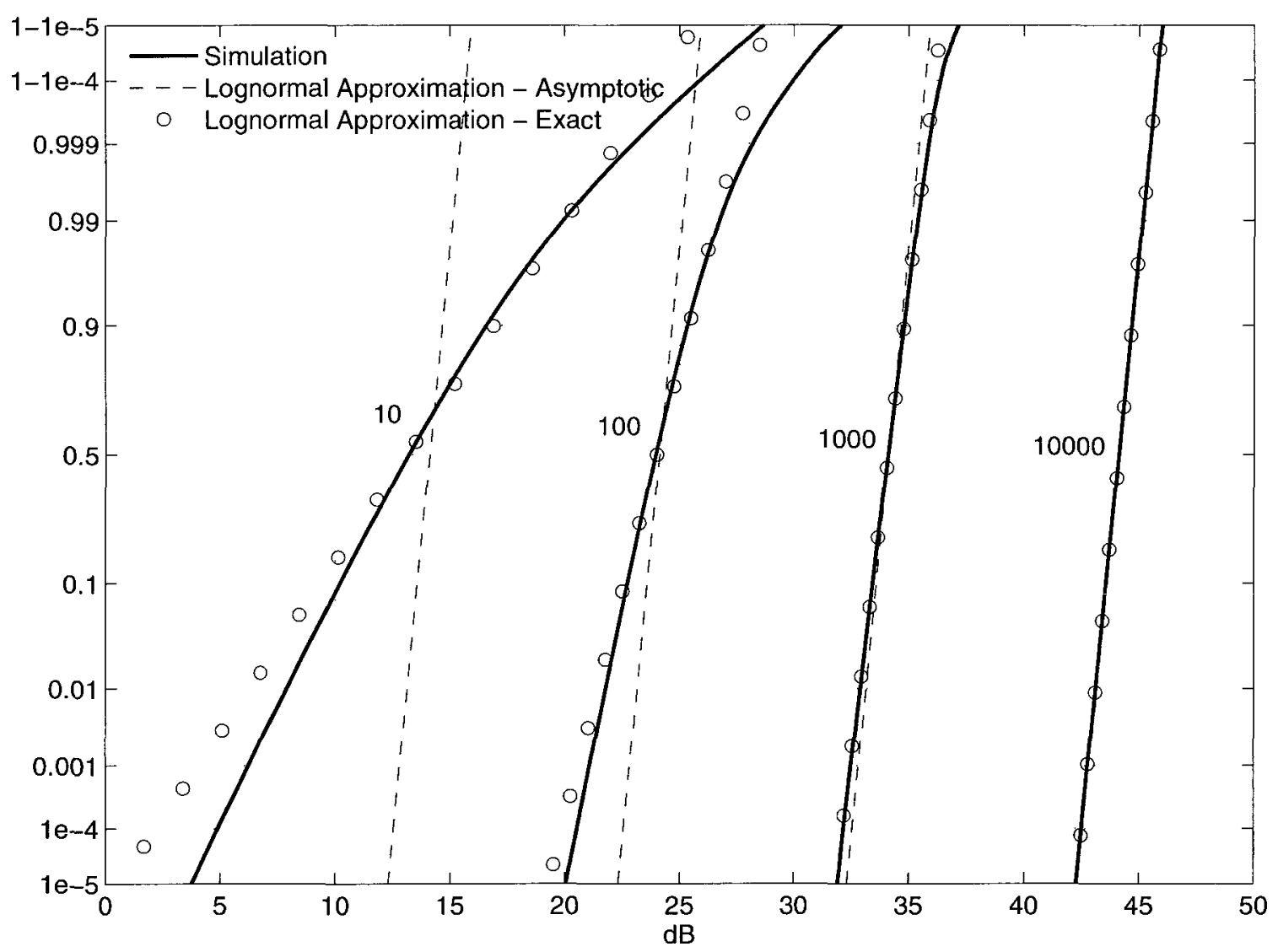

Figure 5.4: SEJLN cdf on lognormal paper, $\sigma=6 \mathrm{~dB}, \rho=0.005$, with varying $N$. 


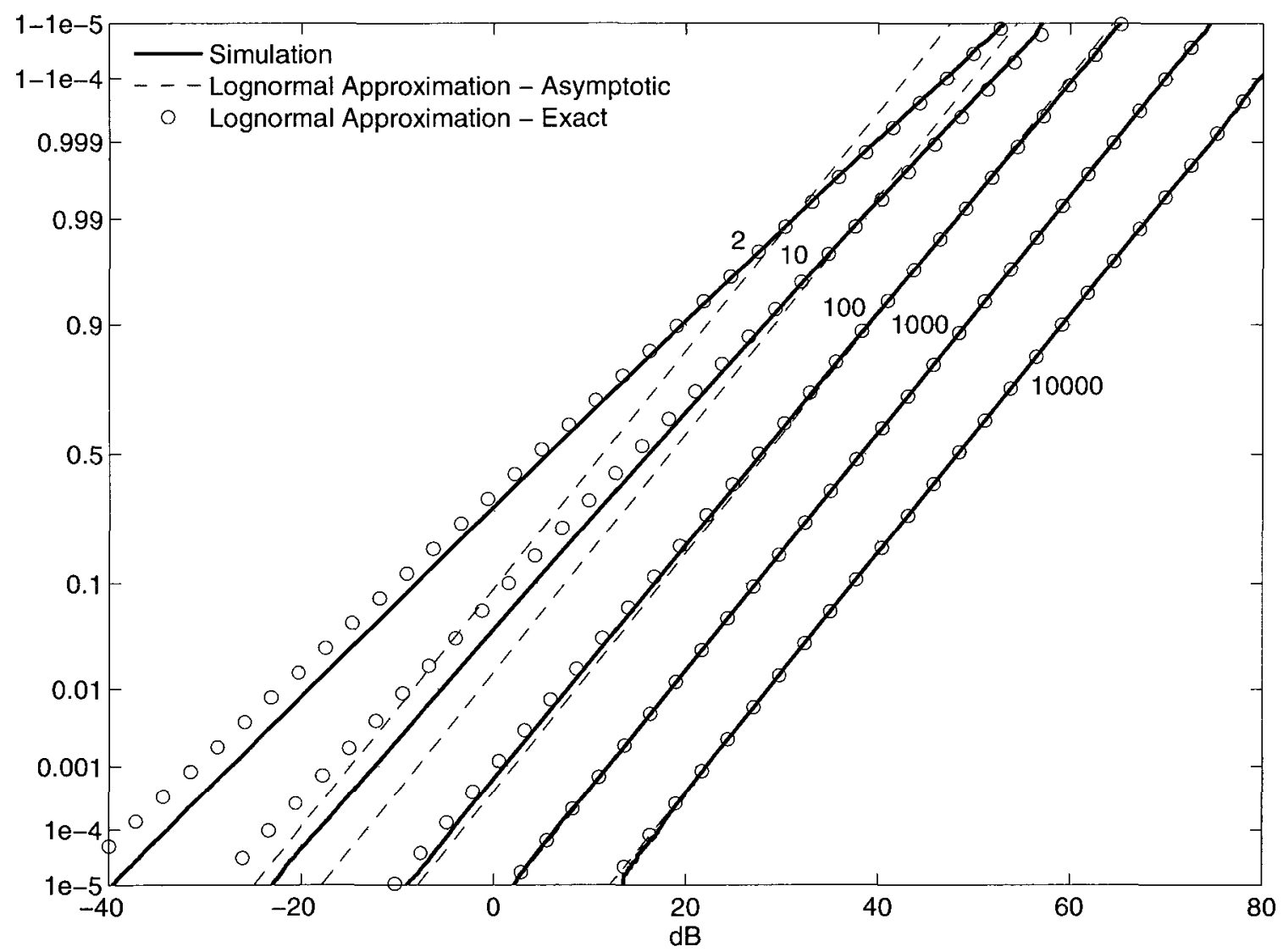

Figure 5.5: SEJLN cdf on lognormal paper, $\sigma=12 \mathrm{~dB}, \rho=0.5$, with varying $N$. 


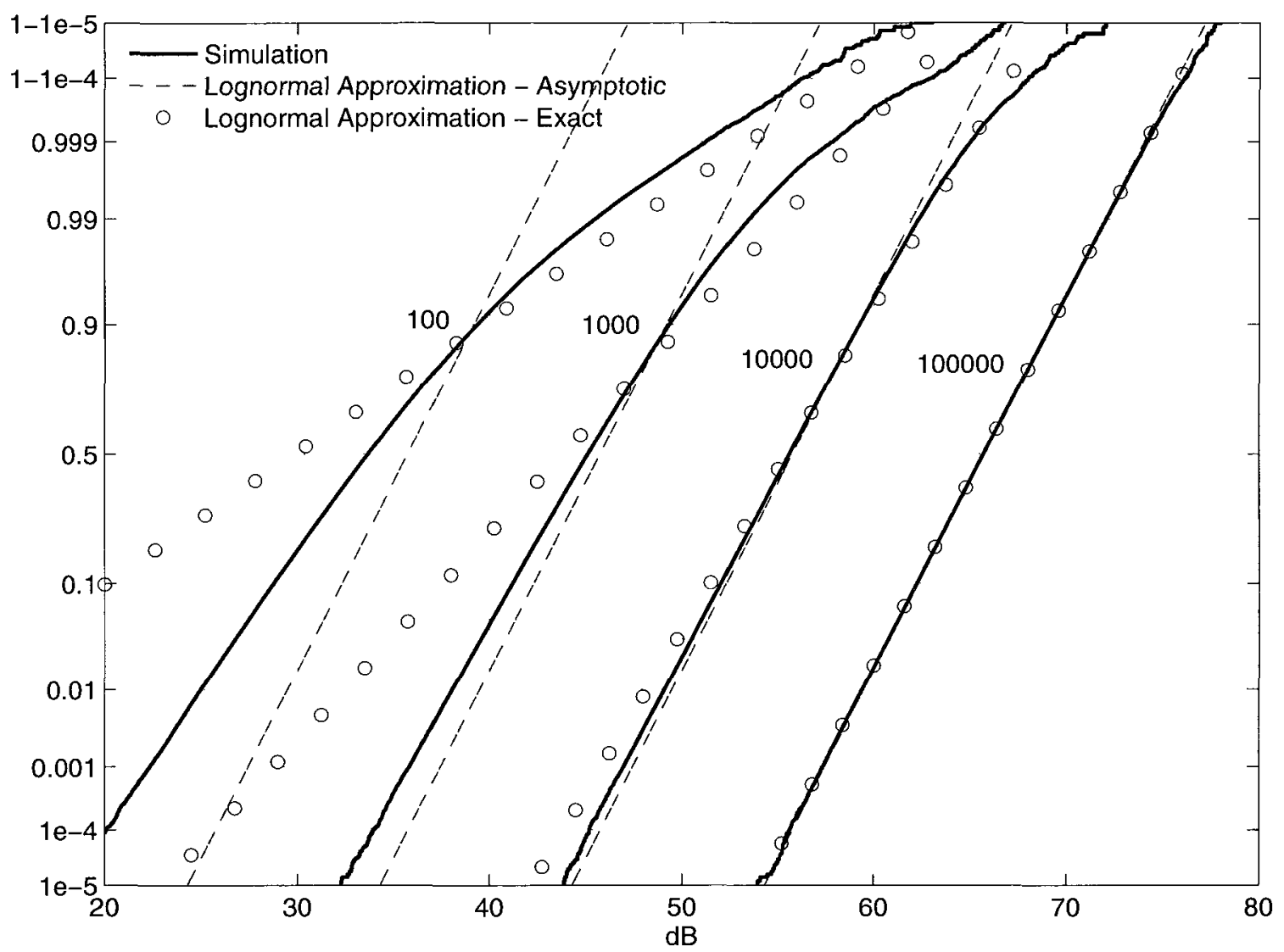

Figure 5.6: SEJLN cdf on lognormal paper, $\sigma=12 \mathrm{~dB}, \rho=0.05$, with varying $N$. 


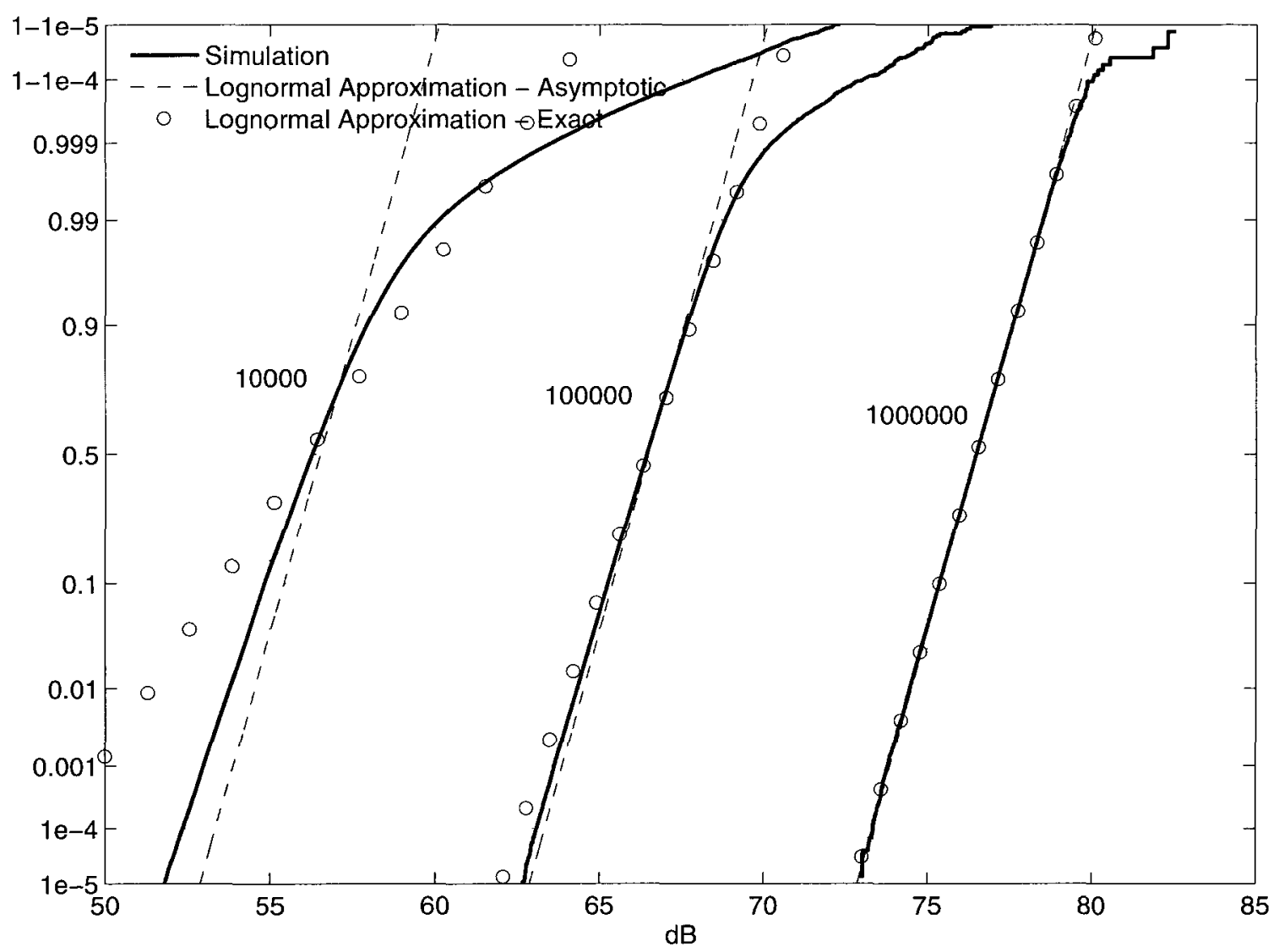

Figure 5.7: SEJLN cdf on lognormal paper, $\sigma=12 \mathrm{~dB}, \rho=0.005$, with varying $N$. 
Table 5.1: Geometric coefficients for each layout in Figure 3.1.

\begin{tabular}{l|lll}
\hline Layout: & $G_{1}$ & $G_{2}-G_{1}^{2}$ & $G_{\text {cor }}$ \\
\hline \hline A : Square Cluster & 5.866 & 0.06763 & 0.5775 \\
B : Gaussian Cluster & 5.617 & 0.04369 & 0.6701 \\
C : Surrounding Ring & 5.738 & 0.1959 & 0.1138 \\
D : Surrounding Thin Ring & 6.215 & $3.331 \cdot 10^{-9}$ & $0.1667 \cong \frac{1}{6}$ \\
\hline
\end{tabular}

Figure 5.7. This is not in contradiction with our result, since we have proved convergence in distributıon, i.e., convergence at every point $x$ (see Definition 14), but not necessarily in the limit behaviour as $x \rightarrow \infty$.

We have thus confirmed the convergence of the SEJLN distribution to the lognormal, and have given a general feel for the rate of this convergence as a function of $\rho$ and $\sigma$. We now verify the accuracy of its application to finding the distribution of $I$.

\subsubsection{Numerical Evaluation of the Geometric Coefficients}

The first step in finding the approximating distribution to that of $I$ is to evaluate the necessary geometric coefficients $G_{1}, G_{2}$, and $G_{\mathrm{cor}}$. The first two coefficients depend only on $g(\vec{r})$, and may therefore be computed directly for any layout, while the last coefficient, $G_{\text {cor }}$, also requires the knowledge of the correlation model. To evaluate $G_{\text {cor }}$, we choose the same model described in Section 3.2.3 and shown in Figure 3.2.

Table 5.1 gives the geometric coefficients for every layout in Figure 3.1. Some details on how to compute these values in practice follow:

- For most coefficients, it is sufficient to evaluate the integrals in (4.17) directly via Riemann integration. However, we find it more judicious to evaluate $G_{1}$ and $G_{2}-G_{1}^{2}$ (which can be interpreted as a mean and variance of $\ln r$ respectively), since this second quantity is often quite small compared to $G_{2}$, and 
the expressions are very sensitive to rounding errors. We find that 1000 uniform integration steps per dimension for $G_{n}$, and 50 steps per dimension for $G_{\mathrm{cor}}$, are sufficient for the integral to converge (given that for a higher number of steps, the integrals remain within 1\%). The computational time for evaluating all three coefficients is around 1.5 seconds, and represents the only computationally-intensive step of our method.

- Finding $G_{\text {cor }}$ in Layout D directly through Riemann integration is not practical, as the ring is so thin that most points on the grid would not land inside it. Instead, when we consider that between $r=499.9$ and $r=500$ we have the $\mathrm{dB}$ ratio $R \cong 0.00087$, which corresponds to a negligible relative difference of 0.00014 in the correlation coefficient when $R_{0}=6 \mathrm{~dB}$, we may approximate $h\left(\vec{r}_{1}, \vec{r}_{2}\right) \cong h(\theta)=\max \left\{1-\theta / \theta_{0}, 0\right\}$. This leads to the approximation

$$
\begin{aligned}
G_{\mathrm{cor}} & \cong\left(\frac{1}{\pi\left(r_{\max }^{2}-r_{\min }^{2}\right)} \int_{r_{\max }}^{r_{\min }} r d r\right)^{2} \cdot \int_{0}^{2 \pi} \int_{0}^{2 \pi} h(\theta) d \theta_{1} d \theta_{2} \\
& =\frac{1}{(2 \pi)^{2}} \int_{0}^{2 \pi} \int_{0}^{2 \pi} h(\theta) d \theta_{1} d \theta_{2}=\frac{1}{2 \pi} \int_{-\pi}^{\pi} \max \left\{1-\frac{\left|\theta_{1}\right|}{\theta_{0}}, 0\right\} d \theta_{1}=\frac{\theta_{0}}{2 \pi}
\end{aligned}
$$

- For uniform annular regions (Layouts $\mathrm{C}$ and $\mathrm{D}$ ), it is simple to evaluate $G_{n}$ analytically. We may rewrite, from (4.17),

$$
\begin{aligned}
G_{n} & =\frac{2}{r_{\max }^{2}-r_{\min }^{2}} \int_{r_{\min }}^{r_{\max }}(\ln r)^{n} r d r \quad \Rightarrow \\
G_{1} & =\frac{r_{\max }^{2}\left(\ln r_{\max }-\frac{1}{2}\right)-r_{\min }^{2}\left(\ln r_{\min }-\frac{1}{2}\right)}{r_{\max }^{2}-r_{\min }^{2}} \\
G_{2} & =\frac{r_{\max }^{2}\left(\left(\ln r_{\max }-1\right) \ln r_{\max }+\frac{1}{2}\right)-r_{\min }^{2}\left(\left(\ln r_{\min }-1\right) \ln r_{\min }+\frac{1}{2}\right)}{r_{\max }^{2}-r_{\min }^{2}} .
\end{aligned}
$$

We now examine how well the resulting lognormal approximation performs, first for clustered, then for non-clustered geometries. 


\subsubsection{Lognormal Approximation to $I$ in a Clustered Layout}

We begin with the clustered layouts, which are A and B in Figure 3.1. Both have a high average shadowing correlation $G_{\text {cor }}>0.5$, and a rather small spread of values

of $r$ (a useful metric for this is $\sqrt{G_{2}-G_{1}^{2}} / G_{1}$ ). We make the approximation that the shadowing spread is constant: $\sigma_{\mathrm{s}}$, and the average pathloss is power law with exponent $\beta$. We may therefore apply the lognormal approximations in (4.8) and (4.9). The results are shown in Figures 5.8-5.11.

If we look back at simulations of the SEJLN cdf for $\rho=0.5$ (Figures 5.2 and 5.5), we observe that the convergence to a lognormal cdf is very good for $N=1000$, and indeed even for smaller $N$. If we assume that the SEJLN problem can be mapped onto the problem of the distribution of $I$, we would expect a similar behaviour. Indeed the convergence is almost exact in the case of Layout A (Figures 5.8 and 5.9), while in the case of Layout B it is somewhat inexact at the lower tail, as we see that the cdf is not quite lognormal. The accuracy of the method rapidly deteriorates when we switch to non-cluster layouts with lower average correlation.

\subsubsection{Lognormal Approximation to $I$ in a Non-Clustered Layout}

We now examine the performance of our approximations in the case of Layout $\mathrm{C}$. The simulations are again performed assuming constant shadowing spread and pathloss exponent. The results in Figure 5.12 confirm that our method does not perform at all well in this case.

Now, recall that the convergence of the SEJLN to a lognormal distribution is proved exactly. When we translate the problem to that of the distribution of $I$, we make two approximations: we assume that each $I_{\imath}$ has a lognormal distribution, and we assume that $\left\{I_{i}\right\}$ is a jorntly lognormal set. It would be interesting to understand the effects of these two approximations to explain the failure of the approximation 


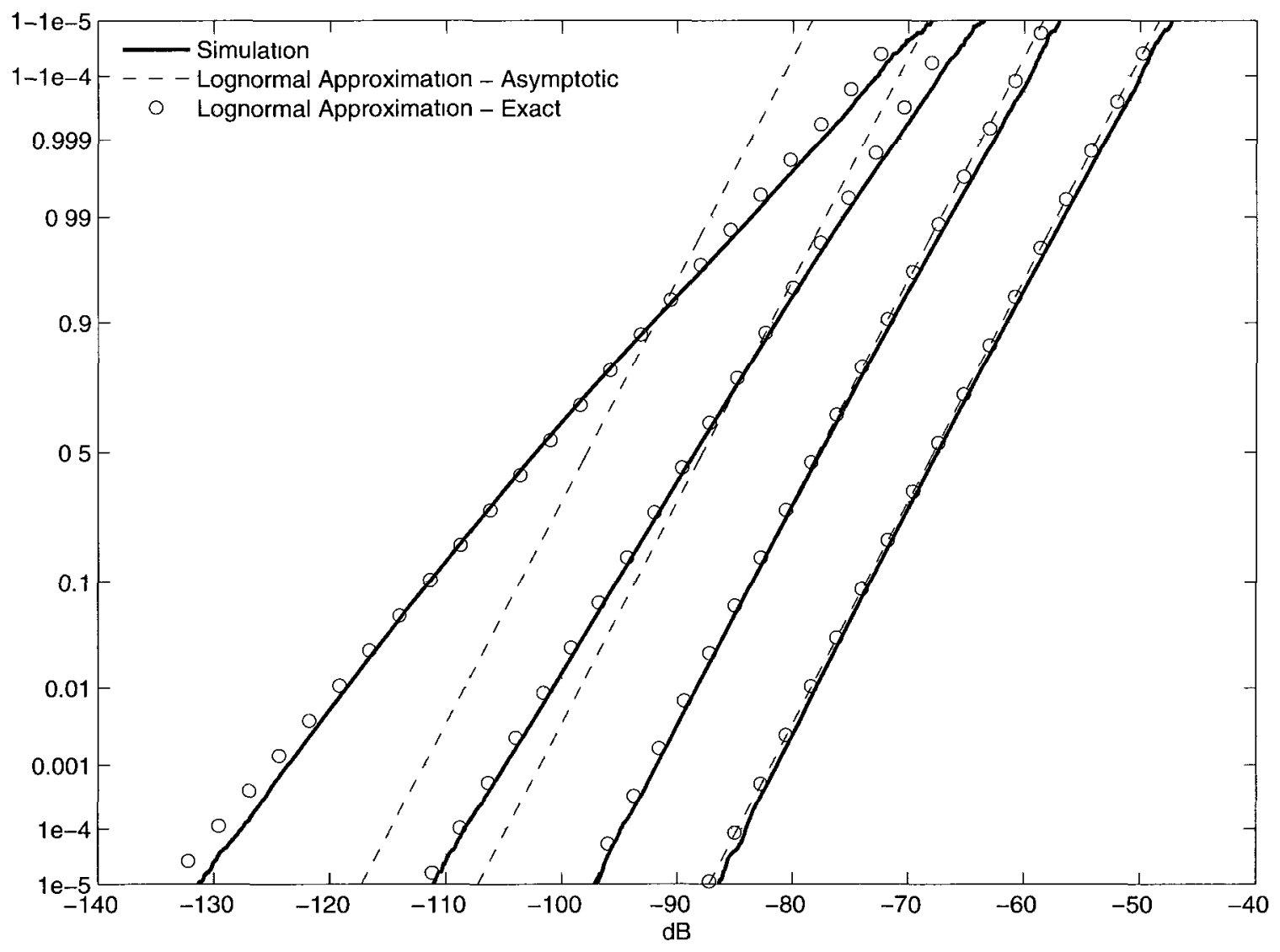

Figure 5.8: Total interference cdf on lognormal paper for Layout $\mathrm{A}$, with $\sigma_{\mathrm{s}}=6 \mathrm{~dB}$, $\beta=4$, and $N=1,10,100,1000$ from left to right. 


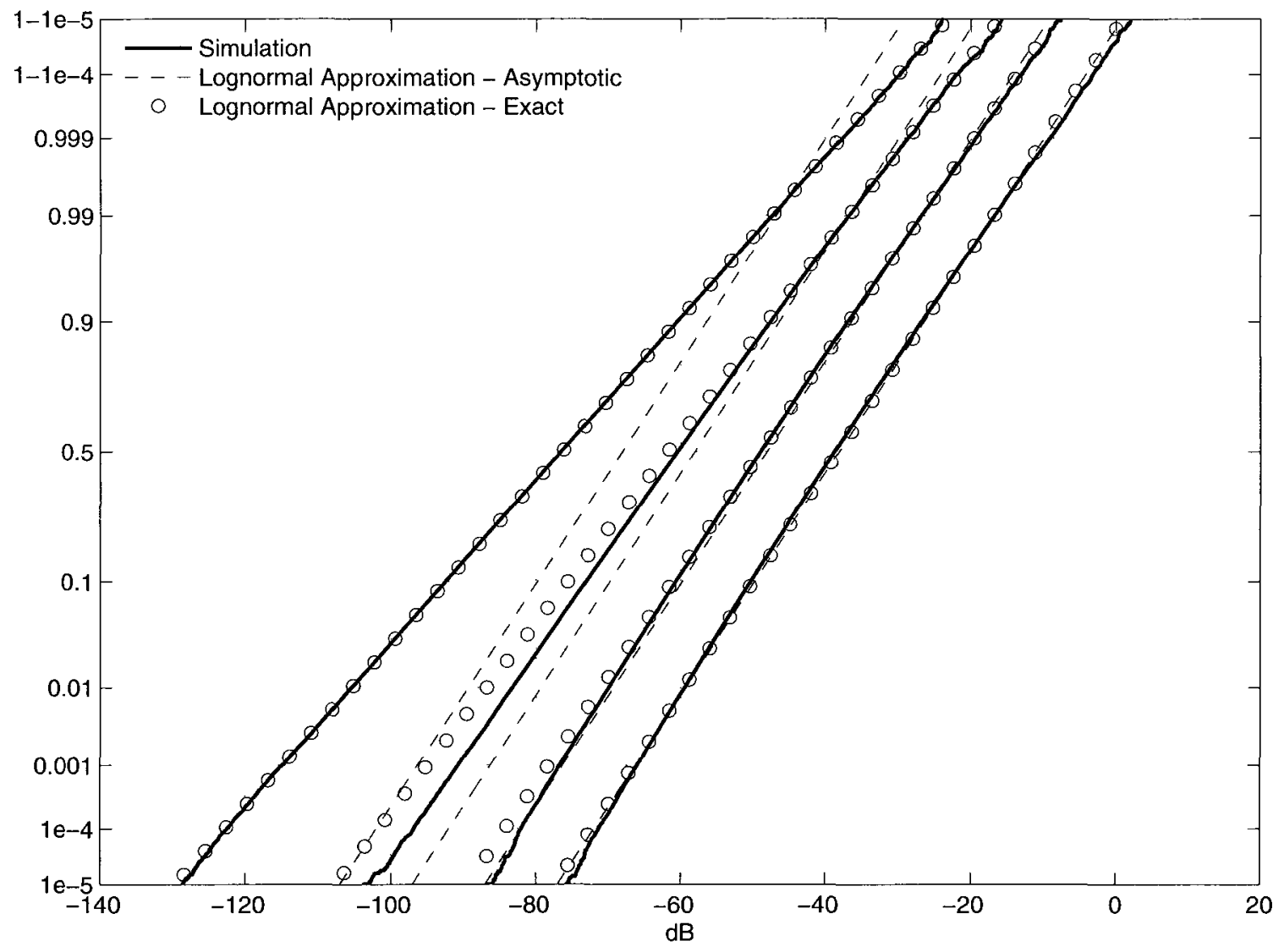

Figure 5.9: Total interference cdf on lognormal paper for Layout A, with $\sigma_{\mathrm{s}}=12 \mathrm{~dB}$, $\beta=3$, and $N=1,10,100,1000$ from left to right. 


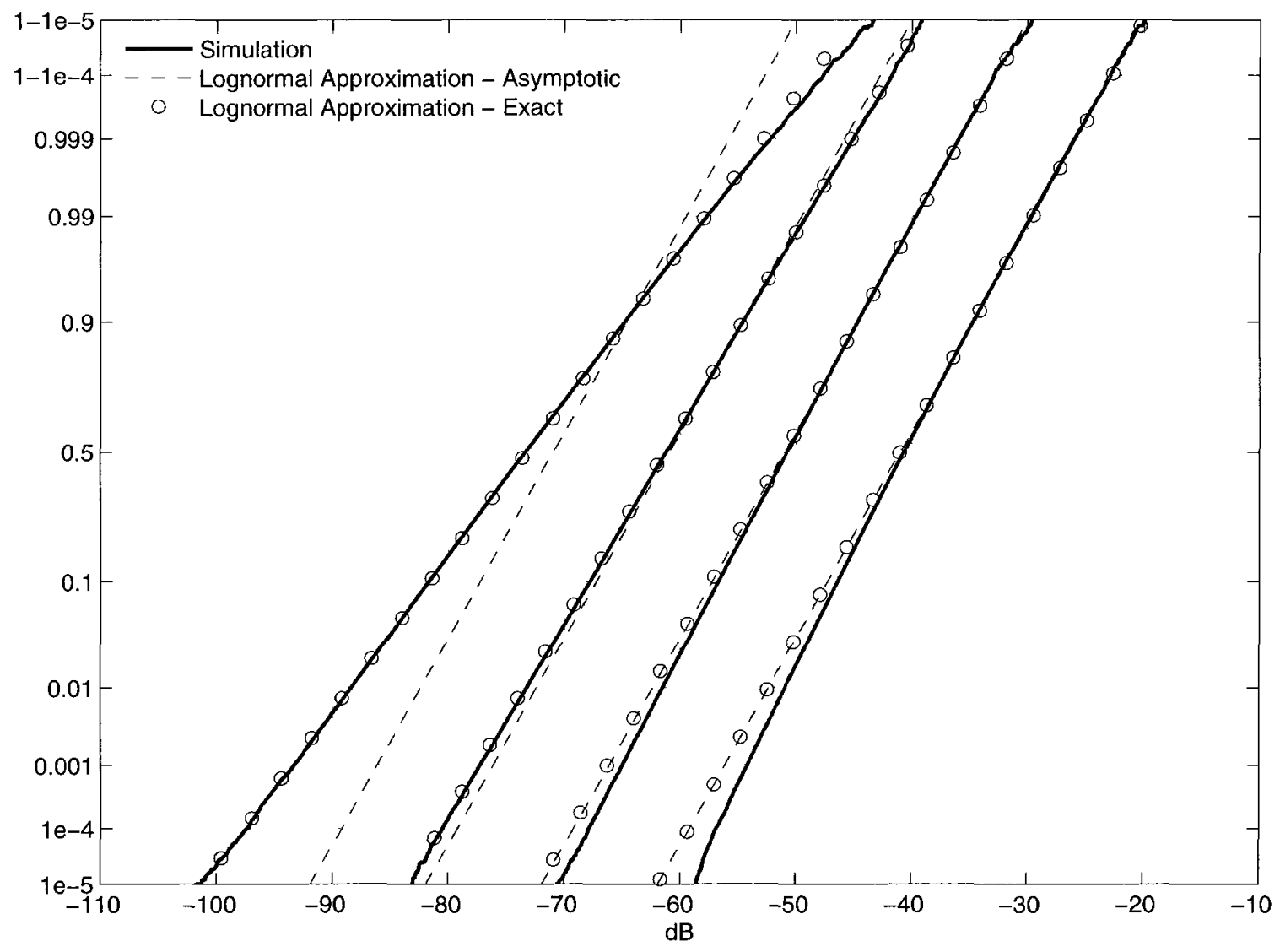

Figure 5.10: Total interference cdf on lognormal paper for Layout B, with $\sigma_{\mathrm{s}}=6 \mathrm{~dB}$, $\beta=3$, and $N=1,10,100,1000$ from left to right. 


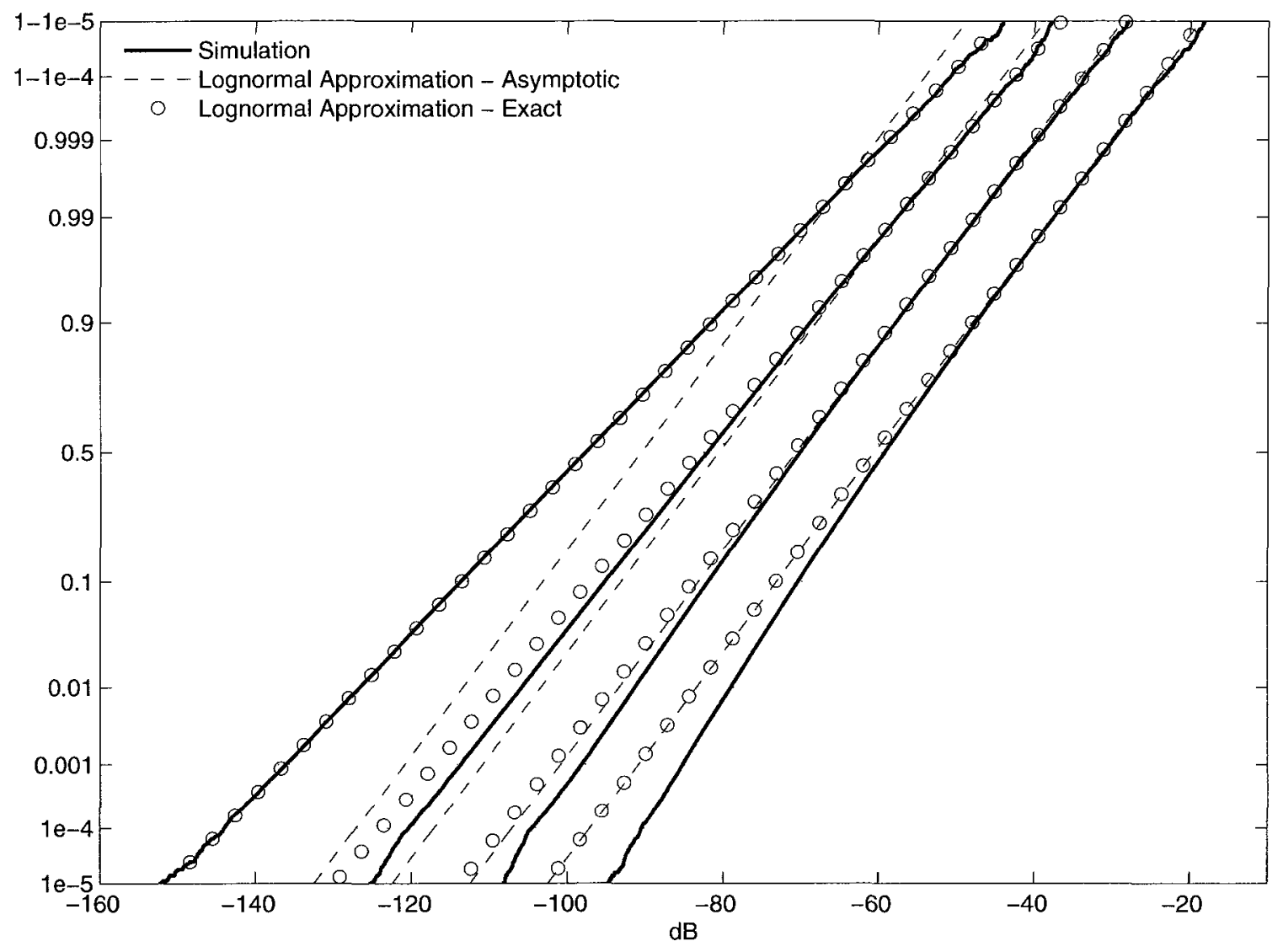

Figure 5.11: Total interference cdf on lognormal paper for Layout B, with $\sigma_{\mathrm{s}}=12 \mathrm{~dB}$, $\beta=4$, and $N=1,10,100,1000$ from left to right. 


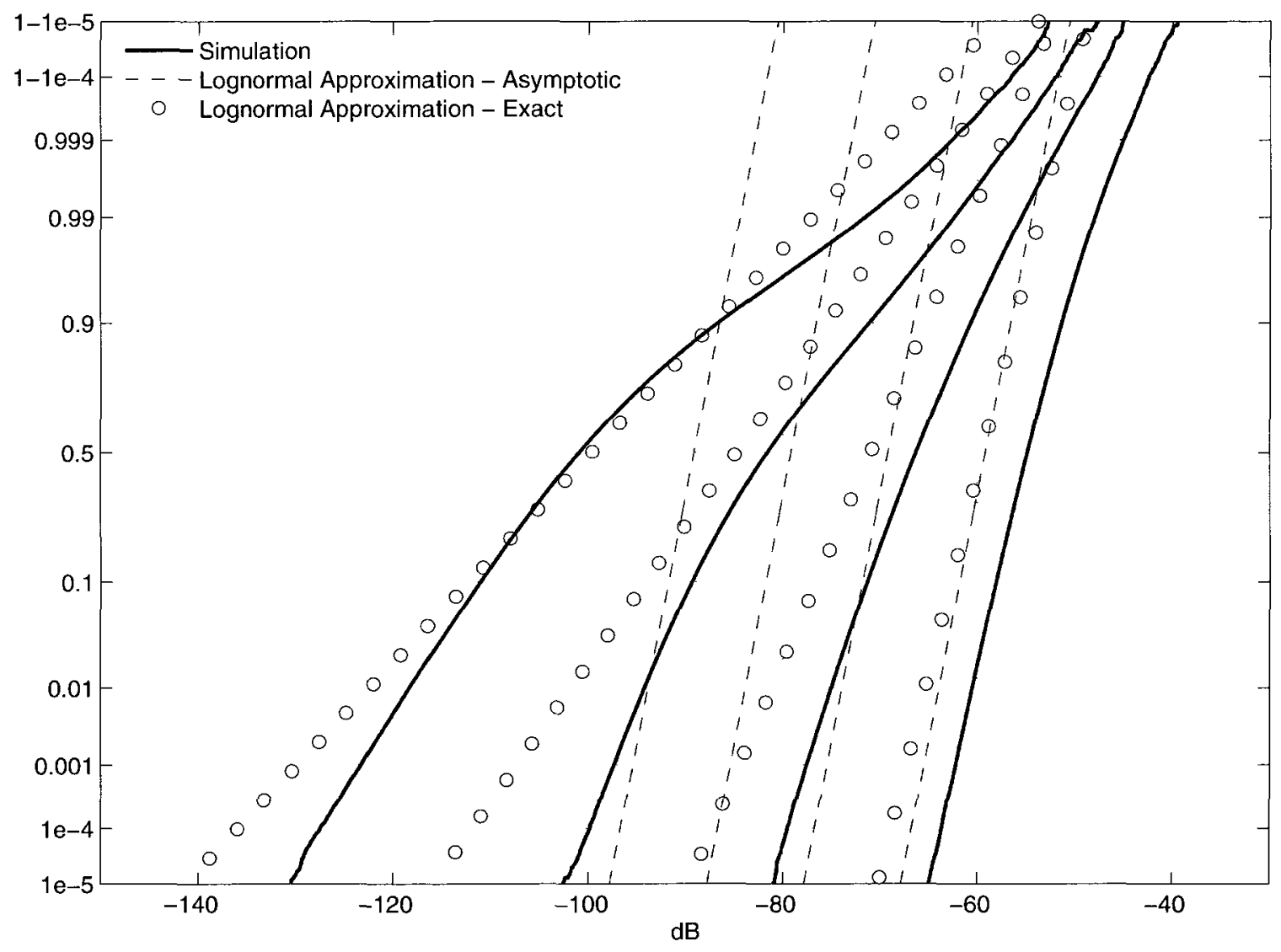

Figure 5.12: Total interference cdf on lognormal paper for Layout $\mathrm{C}$, with $\sigma_{\mathrm{s}}=6 \mathrm{~dB}$, $\beta=4$, and $N=1,10,100,1000$ from left to right. 


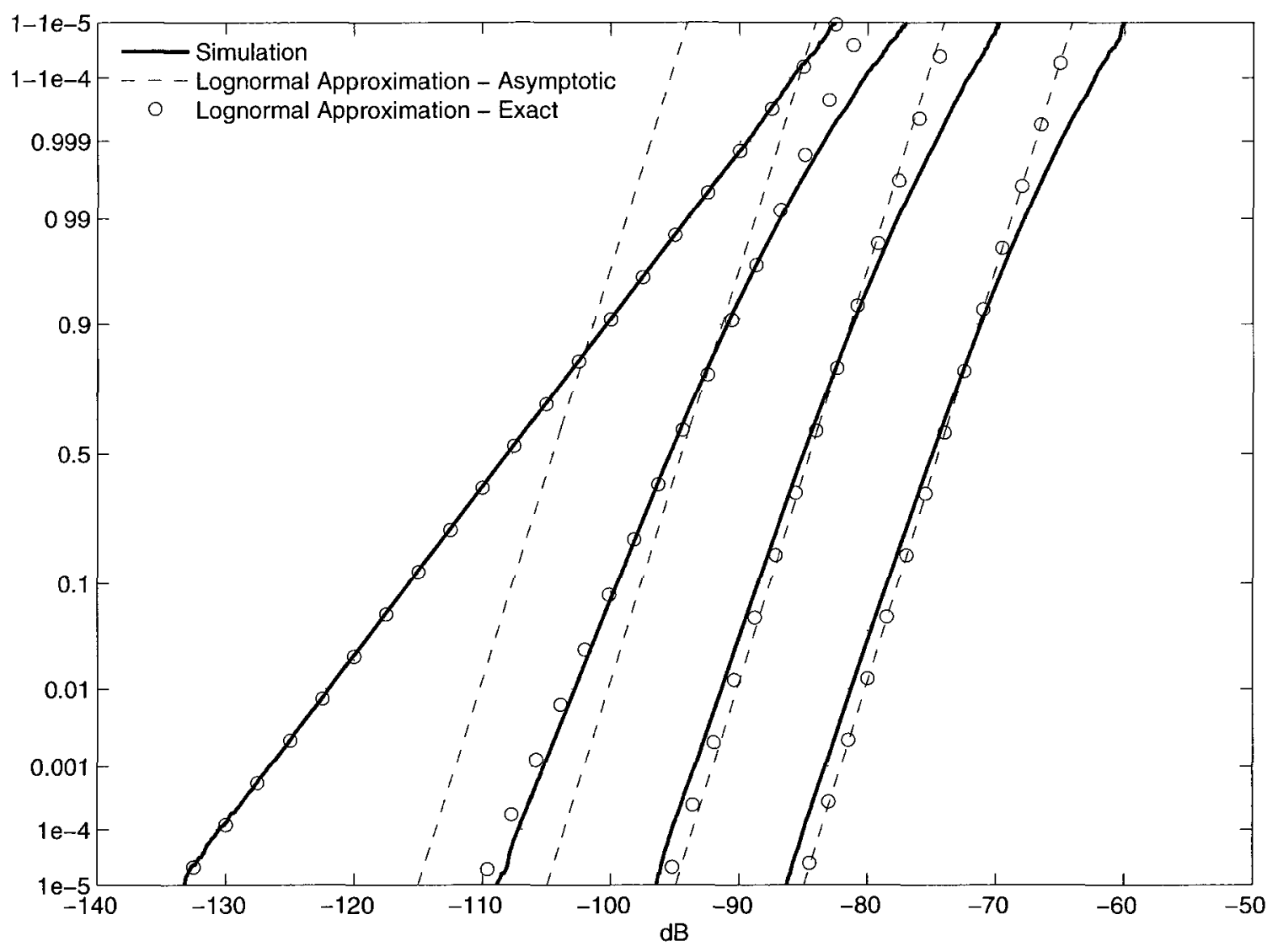

Figure 5.13: Total interference cdf on lognormal paper for Layout $\mathrm{D}$, with $\sigma_{\mathrm{s}}=6 \mathrm{~dB}$, $\beta=4$, and $N=1,10,100,1000$ from left to right. 
in some cases. Firstly, it can be observed from Figure 5.12 for $N=1$ that $I_{\imath}$ is not close to a lognormal. The question is: does jointness even matter? To evaluate this, we consider Layout D (admittedly an artificial case), where we see that $r$ is quasi-constant, and the total interference is well approximated by

$$
I \approx(499.95)^{-\beta} \sum_{\imath=1}^{N} e^{\lambda S_{\imath}}
$$

which, when $\sigma_{\mathrm{s}}$ is constant, is exactly a sum of lognormals (but not jointly lognormal!) formulation. We observe the simulation results in Figure 5.13. In the case of $N=1$, we indeed observe that $I_{\imath}$ is almost perfectly lognormal. However, for $N=1000$, we observe that the distribution of $I$ is not close to lognormal. In this case we have $\sigma[\mathrm{dB}]] \cong \sigma_{\mathrm{s}}=6 \mathrm{~dB}$ and $\rho \cong G_{\text {cor }}=0.1667$. Looking back at Figure 5.3, where $\sigma$ is

the same, and $\rho$ is lower, we still observe almost perfect convergence when $N=1000$; we would therefore expect even better convergence for $\rho=0.1667$, but Figure 5.13 contradicts this.

Because the only difference between (5.5) and the SEJLN problem is jointness, we may conclude that lack of jointness in $\left\{S_{\imath}\right\}$ is a significant impediment to our method. Indeed, we observe in the next section that, in the case of Layout $\mathrm{C}$, while the cdf of $I$ does indeed converge, it is not to a lognormal. A different, multi-faceted approach is required to solve the general problem of the distribution of $I$.

\subsection{Simulating I Rapidly: Combined Simulation-Numerical- Analytical Approach}

The lognormal approximation, combined with the cross-moment matching approach, yields a good approximation for a good subset of large interference problems, but it is clearly inadequate in the general case. The complexity of finding the distribution 
Table 5.2: Simulation settings after calibration.

\begin{tabular}{ll}
\hline Parameter & Value \\
\hline \hline$\underline{\text { Physical Parameters }}$ & \\
$g(\vec{r})$ & Layout C \\
$r_{\min }$ & 50 \\
$r_{\max }$ & 500 \\
$p(r)$ & $r^{-2}(1+r / 150)^{-2}$ \\
$\sigma_{\mathrm{s}}(r)$ & $10(1-\exp (-3 r / 200)) \mathrm{dB}$ \\
$h\left(\vec{r}_{1}, \vec{r}_{2}\right)$ & $\max \left\{1-\theta / \theta_{0}, 0\right\} \cdot \max \left\{1-R / R_{0}, 0\right\}$ \\
$\theta_{0}$ & $60^{\circ}$ \\
$R_{0}$ & $6 \mathrm{~dB}$ \\
$\underline{\text { Simulator Parameters }}$ & \\
$K$ & 1000000 \\
$K_{\vec{r}}$ & 10000 \\
$K_{\mathrm{Ch}}$ & 10000 \\
$D_{\Theta}$ & 12 \\
$D_{\mathrm{R}}$ & 10 \\
$F_{\Theta}$ & 2 \\
$F_{\mathrm{R}}$ & 6 \\
\hline
\end{tabular}


of $I$ in the general case may be too daunting. Simultaneously, the simulation of $I$ for large $N$ is very costly in computational time and memory. In this section, we show how we can approximate the distribution of $I$ well (within $1 \mathrm{~dB}$ ) and quickly (over 1000 times faster for large $N$ ), using a combination of Monte Carlo simulation, image processing techniques, numerical integration, and analytical results.

To this end, we first describe the use of shadowing fields in general, and how they can bring the computational complexity down to $\mathcal{O}(N)$; we also show their particular applicability to the correlation model described in (3.5). We then examine how a judicious reuse of random samples can accelerate the computation by an order of magnitude or more. Finally, we show how to use the equations derived in Section 4.2.3 to extrapolate the distribution of $I$ for very large $N$ from smaller simulations.

The simulations in this section are performed using the parameters in Table 5.2. The simulator parameters are adjusted through a calibration process, which we describe in Section 5.3.4.

\subsubsection{Shadowing Fields}

A shadowing field is a random field in two dimensions, such that it is a Gaussian (given lognormal shadowing) process with a specific auto-correlation function. This auto-correlation is such that, when interferers with positions $\vec{r}_{\imath}$ are placed on the field, and the value of the field at the point $\vec{r}_{\imath}$ is taken as the value of $S_{\imath}$, then $\vec{S} \mid \vec{r}_{1}, \ldots, \vec{r}_{N}$ has the desired correlation matrix $\mathbf{K}$. This can be compared by analogy to gravitational (electric, etc.) fields, where the field gives the acceleration of a mass placed at any point, whether there actually is a mass at that point or not.

The idea of generating shadowing fields has already been explored $[37,38,40,42]$ with correlation functions of the form $h(d)$. We have argued in Section 2.5 that such models may not reflect true shadowing spatial correlation characteristics. Furthermore, correlation as a separable function of $\theta$ and $R$ can be easily simulated by 

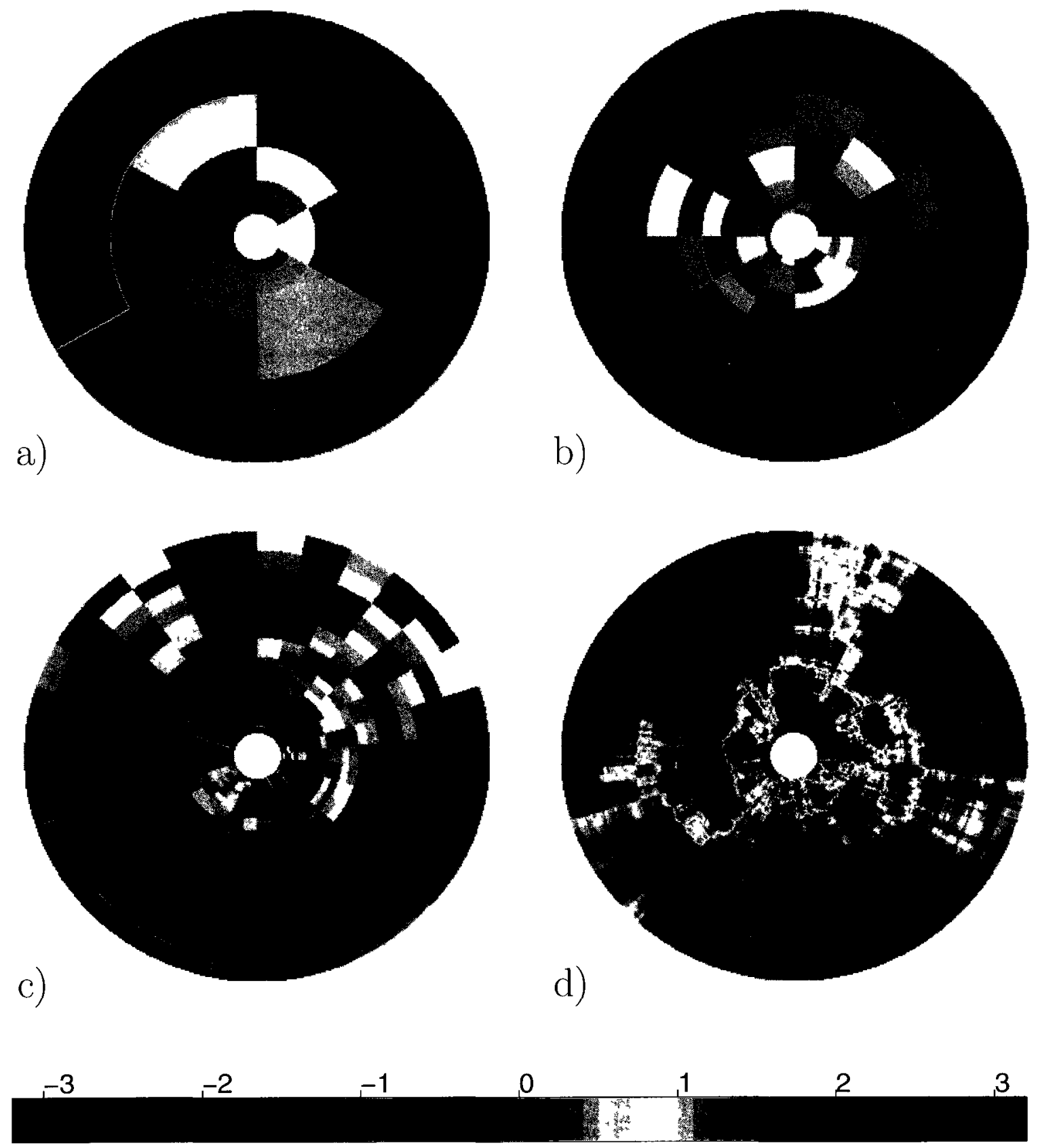

Figure 5.14: Shadowing field realisations for $r_{\min }=50, r_{\max }=500, \theta_{0}=60^{\circ}, R_{0}=$ $6 \mathrm{~dB}$, with increasing resolution: $D_{\Theta}=6 n, D_{\mathrm{R}}=5 n, F_{\Theta}=n, F_{\mathrm{R}}=3 n$, where a) $n=1$, b) $n=2$, c) $n=5$, d) $n=50$. The colour of the areas corresponds to the value of $S_{\imath} / \sigma_{\mathrm{s}}\left(\vec{r}_{\imath}\right)$ therein. 
using a geometric transformation. The accuracy of our method is limited only by the quantisation level.

Consider a random field (a two-dimensional RP) $\bar{M}$ of continuous parameters $(x, y)$. Let the random field be stationary, with an auto-correlation function such that the correlation between the field at two points, $\overline{\mathbf{M}}\left(x_{\imath}, y_{\imath}\right)$ and $\overline{\mathbf{M}}\left(x_{\jmath}, y_{\jmath}\right)$, correspond to the desired shadowing correlation under some transformation.

Consider what we call the $\log$ - polar $^{2}$ transformation:

$$
\begin{aligned}
& \mathcal{T}_{\mathrm{LP}}:(\theta, R) \longmapsto 10^{01 R}(\cos \theta, \sin \theta), \\
& \mathcal{T}_{\mathrm{LP}}:[0,2 \pi] \times\left[10 \log _{10} r_{\min }, 10 \log _{10} r_{\max }\right] \longmapsto\left\{\vec{r}: r_{\min } \leq r \leq r_{\max }\right\}
\end{aligned}
$$

Let us choose the auto-correlation of $\overline{\mathbf{M}}$ as

$$
\begin{aligned}
& \eta_{x}(\xi)= \begin{cases}1-|\xi| / \theta_{0} & |\xi| \leq \theta_{0}, \\
0 & \theta_{0} \leq|\xi| \leq 2 \pi-\theta_{0}, \\
1+(|\xi|-2 \pi) / \theta_{0} & 2 \pi-\theta_{0} \leq|\xi| \leq 2 \pi .\end{cases} \\
& \eta_{y}(v)= \begin{cases}1-|v| / R_{0} & |v| \leq R_{0}, \\
0 & |v|>R_{0},\end{cases} \\
& \mathbb{E}\{\overline{\mathbf{M}}(x+\xi, y+v) \overline{\mathbf{M}}(x, y)\}=\eta_{x}(\xi) \eta_{y}(v),
\end{aligned}
$$

for $\overline{\mathbf{M}}$ defined on $[0,2 \pi] \times\left[10 \log _{10} r_{\min }, 10 \log _{10} r_{\max }\right]$. We find that the field $\overline{\mathbf{M}}$, under transformation $\mathcal{T}_{\mathrm{LP}}$, has the correlation properties of (3.5), i.e.,

$$
\begin{aligned}
& \mathcal{T}_{\mathrm{LP}}:\left(x_{\imath}, y_{\imath}\right) \longmapsto \vec{r}_{\imath}, i=1,2, \ldots \\
& \mathbb{E}\left\{\overline{\mathbf{M}}\left(x_{1}, y_{1}\right) \overline{\mathbf{M}}\left(x_{2}, y_{2}\right)\right\}=h\left(\vec{r}_{1}, \vec{r}_{2}\right) .
\end{aligned}
$$

\footnotetext{
${ }^{2} \mathrm{~A}$ polar representation of certain shadowing fields is suggested in [37].
} 
Therefore, we may write

$$
S_{\imath}=S\left(\vec{r}_{\imath}\right)=\sigma_{\mathrm{s}}\left(r_{\imath}\right) \overline{\mathbf{M}}\left(\mathcal{T}_{\mathrm{LP}}^{-1}\left(\vec{r}_{\imath}\right)\right)
$$

and $S_{\imath}$ 's have the same correlation matrix as in (5.2), while $S(\vec{r})$ is the same stochastic process as described in Section 4.3.

For numerical purposes, $\overline{\mathbf{M}}$ can be approximated by a discrete-parameter matrix $\mathbf{M}_{D_{\Theta} \times D_{\mathrm{R}}}$, with a regularly-spaced quantisation grid along $\theta$ and $R$. Correlation of the form (3.5), triangular in both dimensions, can be obtained by using a uniform square filter $\mathbf{F}_{F_{\Theta} \times F_{\mathrm{R}}}$, ideally choosing $F_{\Theta}$ and $F_{\mathrm{R}}$ so that we have exactly

$$
\begin{aligned}
& F_{\Theta} / D_{\Theta}=\theta_{0} / 2 \pi \\
& F_{\mathrm{R}} / D_{\mathrm{R}}=R_{0} / 10 \log _{10}\left(r_{\max } / r_{\min }\right),
\end{aligned}
$$

with $\mathbf{F}$ equal everywhere to $1 / \sqrt{F_{\Theta} F_{\mathrm{R}}}$.

To obtain the value of the discretised field $\mathbf{M}$ at some point, we must round the coordinates $\mathcal{T}_{\mathrm{LP}}^{-1}\left(\vec{r}_{\imath}\right)$ to the nearest quantisation point. Therefore the algorithm is limited in precision by the finite spatial quantisation. On the other hand, the computational cost of generating one field grows as $\mathcal{O}\left(F_{\Theta} F_{\mathrm{R}} D_{\Theta} D_{\mathrm{R}}\right)=\mathcal{O}\left(D_{\Theta}^{2} D_{\mathrm{R}}^{2}\right)$, and so it is critical to choose the number of quantisation points $D_{\Theta} D_{\mathrm{R}}$ properly to balance precision and computational time. Figure 5.14 shows realisations of the same shadowing fields at different resolutions.

Shadowing fields have the additional advantages of requiring only $\mathcal{O}(N)$ memory (rather than $\mathcal{O}\left(N^{2}\right)$ for matrix factorisation), and being able to accommodate interferer mobility easily: indeed, while matrix factorisation only gives shadowing values at the $N$ specified locations, shadowing fields give the value of the (potential) shadowing everywhere. This is also useful when $N$ is uncertain, random, or variable. 


\subsubsection{Efficient Filtering for Separable Triangular Correlations}

The choice of the correlation model (3.5) is particularly fortunate from the point of view of computational efficiency, because it benefits from two properties: separability and a triangular shape.

\subsubsection{Separability}

The nature of the correlation model (3.5) is such that it can be expressed as the product of a function of $\theta$ and a function of $R$. It follows [60] that the resulting twodimensional process in the $\theta-R$ plane is also separable. It can therefore be simulated by filtering over each dimension separately, which reduces the general filtering cost from $\mathcal{O}\left(F_{\Theta} F_{\mathrm{R}} D_{\Theta} D_{\mathrm{R}}\right)$ (as in, e.g., [38]) to $\mathcal{O}\left(\left(F_{\Theta}+F_{\mathrm{R}}\right) D_{\Theta} D_{\mathrm{R}}\right)$.

\subsubsection{Optimised Box Filters}

The triangular form in $\theta$ and $R$ of the correlation expression in (3.5) requires the use of rectangular (box) filters applied to a white Gaussian process. Computationally this is very efficient, as the filtering requires no multiplications. Additionally, it can be implemented even more efficiently [163], with the number of additions now approximately $2 D_{\ominus}$ and $2 D_{\mathrm{R}}$ in each respective dimension, rather than $\left(F_{\ominus}-1\right) D_{\ominus}$ and $\left(F_{\mathrm{R}}-1\right) D_{\mathrm{R}}$ respectively. This is due to the fact that adjacent outputs of a box filter differ only by two input values. This makes the total computation cost for one field realisation $\mathcal{O}\left(D_{\Theta} D_{\mathrm{R}}\right)$, which is independent of the filter size and hence of the correlation distances $\theta_{0}$ and $R_{0}$.

\subsubsection{Optimised Shadow Fields Algorithm}

A general shadowing fields algorithm would have such a form:

Basic Shadowing Field Algorithm 
Ensure: The histogram of $I[k]$ approximates the pdf of $I$.

$$
\text { for } \begin{aligned}
k & =1 \text { to } K \text { do } \\
\text { for } i & =1 \text { to } N \text { do } \\
\vec{r}_{\imath} & \Leftarrow i . \text { r.d. random from } g(\vec{r})
\end{aligned}
$$

end for

$$
\begin{aligned}
& \mathbf{M} \Leftarrow \text { shadowing field realisation }{ }^{\dagger} \\
& \text { for } i=1 \text { to } N \text { do } \\
& S_{\imath} \Leftarrow \sigma_{\mathrm{s}}\left(r_{\imath}\right) \mathbf{M}\left[\mathcal{T}_{\mathrm{LP}}^{-1}\left(\vec{r}_{\imath}\right)\right] \text { (nearest index) }
\end{aligned}
$$

end for

$$
I[k] \Leftarrow \sum_{\imath=1}^{N} p\left(r_{\imath}\right) e^{\lambda S_{\imath}}
$$

end for

Now the correlation model (3.5) benefits from separability in the $R$ and $\theta$ domains, as well as from the triangular form of both auto-correlations. It is also important to remember to wrap the field in the $\theta$ direction so as to ensure angular continuity. These elements produce the following detailed implementation:

\section{${ }^{\dagger}$ FAst Shadowing Field Generation}

Ensure: $\mathbf{M}$ is Gaussian, correlated approximately as (3.5).

$\mathrm{Z}_{D_{\Theta} \times\left(D_{\mathbf{R}}+F_{\mathrm{R}}-1\right)} \Leftarrow$ i.i.d. random $\mathcal{N}(0,1)$

Initialise a temporary matrix $\mathbf{W}_{D_{\Theta} \times\left(D_{\mathrm{R}}+F_{\mathrm{R}}-1\right)}$

for $m=1$ to $D_{\mathrm{R}}+F_{\mathrm{R}}-1$ do

$\mathbf{W}[1, m] \Leftarrow \sum_{n=1}^{F_{\ominus}} \mathbf{Z}[n, m]$

end for

$$
\begin{aligned}
& \text { for } n=1 \text { to } D_{\Theta}-1 \text { do } \\
& \begin{array}{l}
n^{*} \Leftarrow\left(n+F_{\Theta}-1\right) \bmod D_{\Theta}+1 \\
\text { for } m=1 \text { to } D_{\mathrm{R}}+F_{\mathrm{R}}-1 \mathbf{d o} \\
\quad \mathbf{W}[n+1, m] \Leftarrow \mathbf{W}[n, m]-\mathbf{Z}[n, m]+\mathbf{Z}\left[n^{*}, m\right]
\end{array}
\end{aligned}
$$


end for

end for

for $n=1$ to $D_{\Theta}$ do

$\mathbf{M}[n, 1] \Leftarrow \sum_{m=1}^{F_{\mathrm{R}}} \mathbf{W}[n, m]$

end for

for $m=1$ to $D_{\mathrm{R}}-1$ do

for $n=1$ to $D_{\Theta}$ do

$\mathbf{M}[n, m+1] \Leftarrow \mathbf{M}[n, m]-\mathbf{W}[n, m]+\mathbf{W}\left[n, m+F_{\mathrm{R}}\right]$

end for

end for

\subsubsection{Reusing Random Samples}

Converting the simulation algorithm from matrix factorisation to shadowing fields can bring a great gain in simulation time for large $N$. We may, however, augment these gains by using an additional technique, based on reusing random samples, that can be applied to both algorithms to greatly decrease their computational time at a negligible cost in accuracy.

We begin with the observation that both algorithms, while quite different, are also fundamentally similar: both require the generation of $N K$ positions from $g(\vec{r})$, and also the generation of a large set of independent Gaussian RVs. These RVs are then linearly combined to obtain the required correlation structure, after which we may compute $I_{i}$ and $I$ according to (3.6). We further observe that the generation of the independent Gaussians and that of the interferer positions are two separate processes.

This leads to the idea of generating fewer random quantities of both kinds, and pairing them in different combinations so as to achieve a similar amount of randomness as if each realisation was different. We call $K_{\vec{r}}$ the number of times the positions of 
the interferers are generated, for a total sample size of $N K_{\vec{r}}$ from $g(\vec{r})$. We also call $K_{\mathrm{Ch}}$ the total number of channel realisations generated, though this has a somewhat different meaning in both algorithms. We impose the following conditions:

$$
\begin{aligned}
K / K_{\vec{r}} & \in \mathbb{N}^{*}, \\
K / K_{\mathrm{Ch}} & \in \mathbb{N}^{*}, \\
K_{\vec{r}} K_{\mathrm{Ch}} / K & \in \mathbb{N}^{*} .
\end{aligned}
$$

An important implementation consideration is which of the two, positions or Gaussians, to generate first, as these need to be stored in memory during the simulation to allow for reuse. Let us examine the details of implementing reuse for each algorithm separately.

\subsubsection{Reuse in Matrix Factorisation}

Matrix factorisation requires the generation and factorisation of $K_{\vec{r}} N \times N$ matrices, but the generation of only $N \times K_{\mathrm{Ch}}$ independent Gaussians. It is therefore more judicious, for large $N$, to generate the Gaussian RVs first and store them for reuse, and then to generate and discard the interferers' positions and correlation matrices one by one. This can be implemented as follows:

\section{Matrix FaCtorisation Algorithm with Reuse}

Ensure: The histogram of $I[k]$ approximates the pdf of $I$.

$$
\begin{aligned}
& \text { for } k=1 \text { to } K_{\mathrm{Ch}} \text { do } \\
& \text { for } n=1 \text { to } N \text { do } \\
& Z_{n}[k] \Leftarrow i . i . d \text {. random } \mathcal{N}(0,1)
\end{aligned}
$$

end for

end for

for $k=1$ to $K_{\vec{r}}$ do 


$$
\begin{aligned}
& \text { for } i=1 \text { to } N \text { do } \\
& \quad \vec{r}_{i} \Leftarrow i . i . d \text {. random from } g(\vec{r}) \\
& \text { end for } \\
& \text { for } i=1 \text { to } N \text { do } \\
& \quad \bar{K}[i, i] \Leftarrow 1 \\
& \quad \text { for } j=1 \text { to } i-1 \text { do } \\
& \quad \bar{K}[i, j]=\bar{K}[j, i] \Leftarrow h\left(\vec{r}_{i}, \vec{r}_{j}\right) \\
& \quad \text { end for } \\
& \text { end for } \\
& \overline{\mathbf{C}} \Leftarrow \sqrt[*]{\overline{\mathbf{K}}} \\
& \text { for } l=1 \text { to } K / K_{\vec{r}} \operatorname{do} \\
& k^{*} \Leftarrow l+(k-1) K / K_{\vec{r}} \\
& l^{*} \Leftarrow\left(k^{*}-1\right) \bmod K_{\mathrm{Ch}}+1 \\
& \vec{S} \Leftarrow \operatorname{diag}\left(\sigma_{\mathrm{s}}\left(\vec{r}_{1}\right), \ldots, \sigma_{\mathrm{s}}\left(\vec{r}_{N}\right)\right) \cdot \vec{Z}\left[l^{*}\right] \cdot \overline{\mathbf{C}} \\
& I\left[k^{*}\right] \Leftarrow \sum_{i=1}^{N} p\left(r_{i}\right) e^{\lambda S_{i}} \\
& \text { end for } \\
& \text { end for }
\end{aligned}
$$

Here the calculation of $l^{*}$ establishes the way reused random values are assigned to each other. Of course, there are many equivalent such mappings, as all the random values are iid, and thus exchangeable.

\subsubsection{Reuse in Shadowing Fields}

Applying reuse in shadowing fields requires a reversed approach: because the shadowing fields algorithm is asymptotically faster than matrix factorisation, it is better to first generate the channel realisations (namely, the fields), whose memory cost is $D_{\Theta} D_{\mathrm{R}} K_{\mathrm{Ch}}$, and does not depend on $N$. A possible implementation is: 


\section{Basic Shadowing Field Algorithm with Reuse}

Ensure: The histogram of $I[k]$ approximates the pdf of $I$.

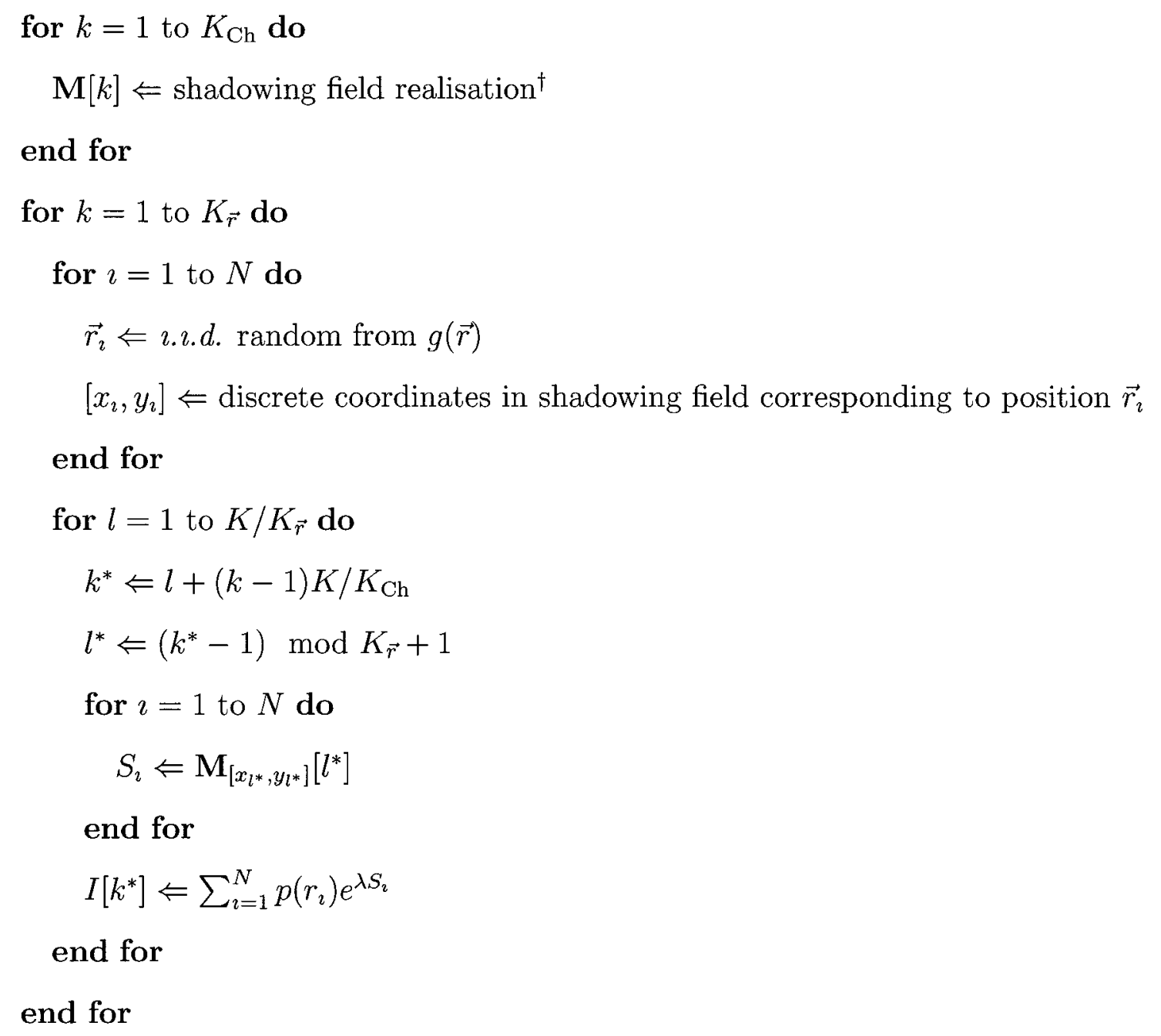

\subsubsection{Simulator Calibration}

In order to apply shadowing fields and random sample reuse to a simulation problem, decisions need to be made regarding some parameters that did not exist in the initial problem statement, but are required to use the approximating methods. We call this process calibration, and require that the simulation parameters are such that the resulting approximating distributions do not differ in most places by more than $1 \mathrm{~dB}$ 
from the simulations with matrix factorisation and no reuse, which are considered exact. While increasing these parameters gives more accurate results, simulation time suffers, and as such a compromise must be struck.

The first step is to choose a suitable resolution for the shadowing fields. For simplicity, we change $D_{\Theta}$ and $D_{\mathrm{R}}$ jointly and proportionally, and allow only values that give integer values of $D_{\Theta} \theta_{0} / 2 \pi$ and $D_{\mathrm{R}} R_{0} / 10 \log _{10}\left(r_{\max } / r_{\min }\right)$. In the case of the parameters chosen in Table 5.2, we may set $D_{\Theta}=6 n, D_{\mathrm{R}}=5 n$, which gives $F_{\Theta}=n$ and $F_{\mathrm{R}}=3 n$, with $n \in \mathbb{N}^{*}$.

Also, Figure 5.14 shows the effect of increasing $n$ on the appearance of realisations of shadowing fields, showing greater detail with increasing $n$, but at a cost of computational time $\mathcal{O}\left(n^{2}\right)$. In Figure 5.15, we observe the effect of the resolution on the distribution of $I$ simulated using shadowing fields, with $n=1,2,3$. First we observe that, for $N=1$, there is no significant difference between any of the simulations, since there is in fact no correlation, and we have merely shown that all algorithms produce the same marginal distribution for $I_{2}$. For $N=10$, we observe some significant distortion in the lower tail, which seems little affected by changing the field resolution. For higher $N$, however, we clearly see an improvement with increasing resolution: while $n=1$ gives rather poor results in both tails, $n=2,3$ give very accurate results across the whole range of values. Thus we consider $n=2$ sufficient at this point, which leads to the simulation parameters listed in Table 5.2.

The next step is choosing the amount of reuse for both channel and interferer position realisations. We set $K_{\mathrm{Ch}}=K_{\vec{r}}$ for simplicity, and can subsequently define a reuse factor $m=K / K_{\vec{r}}=K / K_{\mathrm{Ch}}$. We now examine the effect of the reuse factor on the distortion of the simulated distribution of $I$. Figures 5.16 and 5.17 show the effect of reuse on matrix factorisation and shadowing fields, respectively. We first observe that reusing random samples causes distortion in the far tails. This can be predicted from the fact that far tails are associated with rare events, whose 


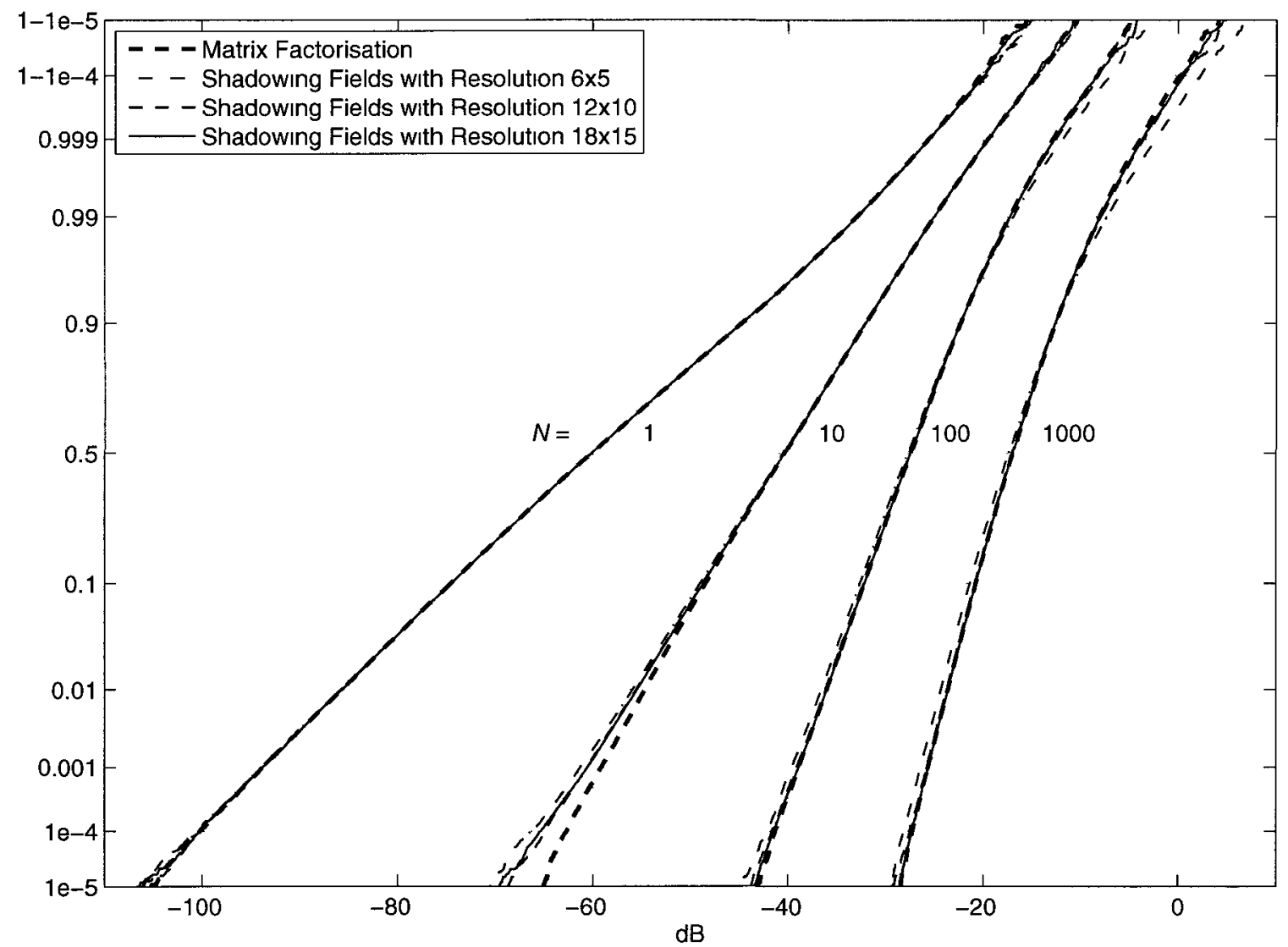

Figure 5.15: Calibration of field resolution parameters $D_{\Theta}=6 n$ and $D_{\mathrm{R}}=5 n$, and the resulting simulated cdfs of $I$. 


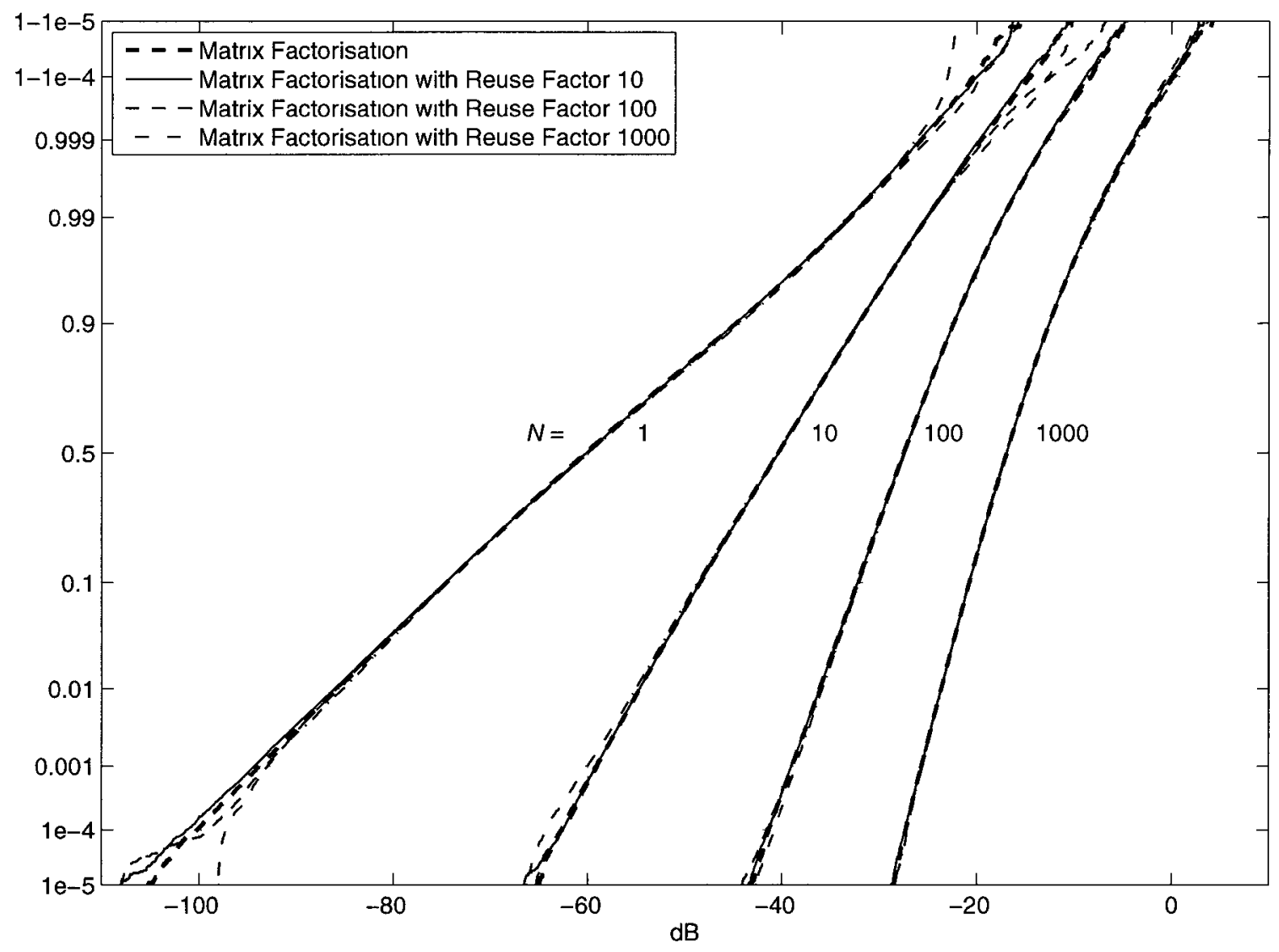

Figure 5.16: Effect of random sample reuse in matrix factorisation algorithm on the cdf of $I . K=1000000, K_{\vec{r}}=K_{\mathrm{Ch}}=K / m$. 


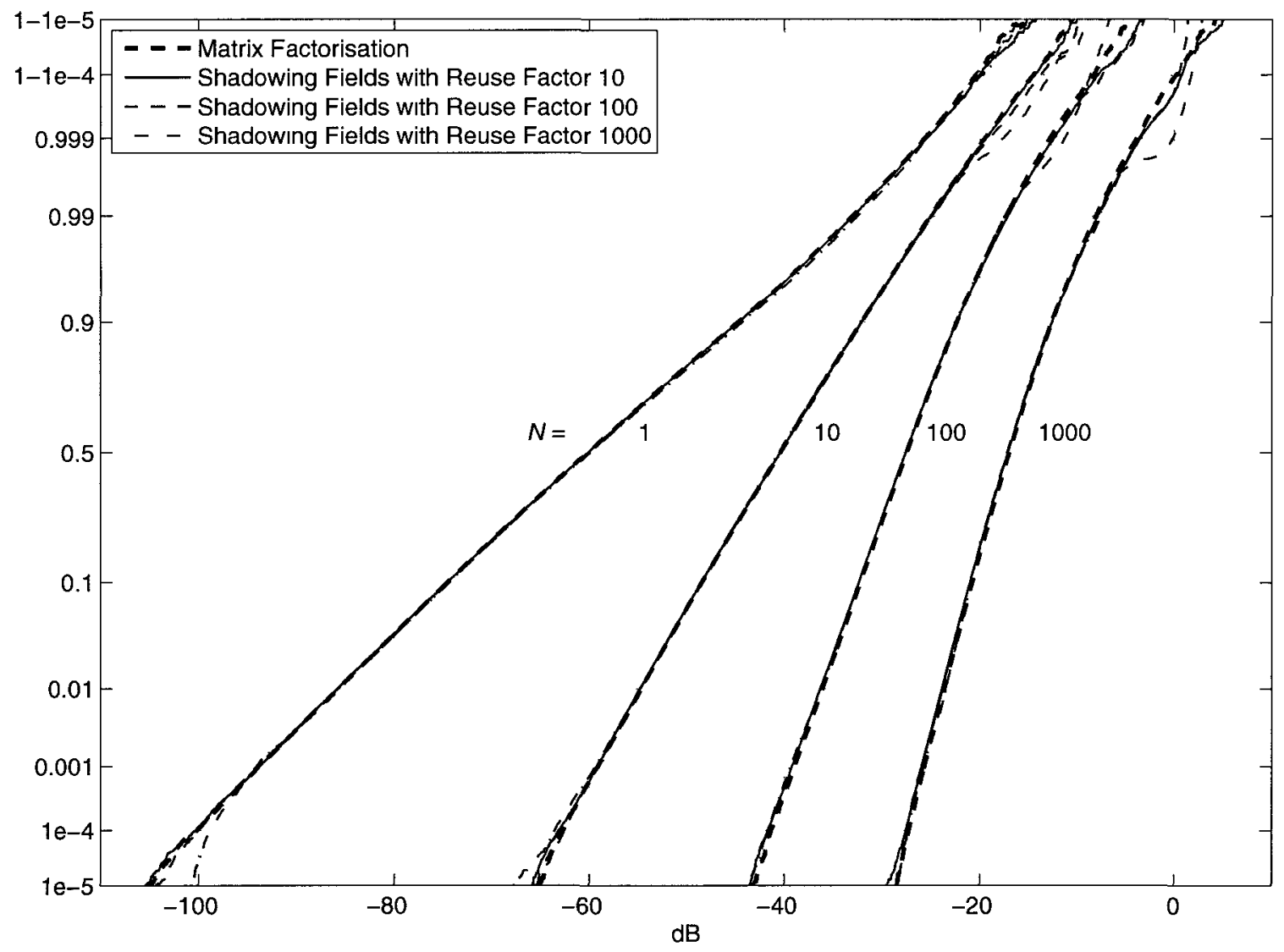

Figure 5.17: Effect of combining random sample reuse with calibrated $\left(D_{\Theta}=\right.$ $\left.12, D_{\mathrm{R}}=10\right)$ shadowing fields algorithm on the cdf of $I . K=1000000, K_{\vec{r}}=$ $K_{\mathrm{Ch}}=K / m$. 
occurrence is seriously affected by repetition of random values. We also observe that the maximum possible reuse of $m=1000$ causes too much distortion in certain cases, but $m=100$ gives very reasonable accuracy. We thus choose $K_{\mathrm{Ch}}=K_{\vec{r}}=10000$.

We also observe that the performance with reuse is usually worse for smaller $N$ (=10), and improves with increasing $N$. This can be interpreted from the fact that, given a particular $K_{\vec{r}}$ and $K_{\mathrm{Ch}}$, the number of total independent RVs generated by the simulator increases with $N$, and thus there is more "randomness" in the system, which leads to more accurate distributions. This trend is encouraging, as it confirms that our approach is well-suited for large $N$.

\subsubsection{Time Performance Comparison}

Once we have calibrated the simulator to produce accurate results, we perform a comparison of the computation time against $N$ required for a simulation using Cholesky factorisation versus using shadowing fields as described previously. Additionally, we show the performance of both algorithms with reuse of random samples (each sample is reused $K / K_{\vec{r}}=K / K_{\mathrm{Ch}}=100$ times). The performances of all four algorithms are shown in Figure 5.18.

The first observation to make is that shadow fields always outperform matrix factorisation, except for $N=1$, where there is in fact no correlation and generating shadow fields is entirely redundant. Furthermore, we observe that for $N$ beyond about 20, the time required for Cholesky factorisation increases drastically, and takes on a sharper trend than shadowing fields, proving that the shadowing fields algorithm always eventually outperforms matrix factorisation. Indeed, as can be observed from the algorithm, shadowing fields require an initial investment to generate the field realisations, after which the computational cost is linear in $N$, which is confirmed by the asymptotic behaviour of both shadowing fields algorithms. On the other hand, both Cholesky factorisation curves show an asymptotic trend between $\mathcal{O}\left(N^{2}\right)$ and 


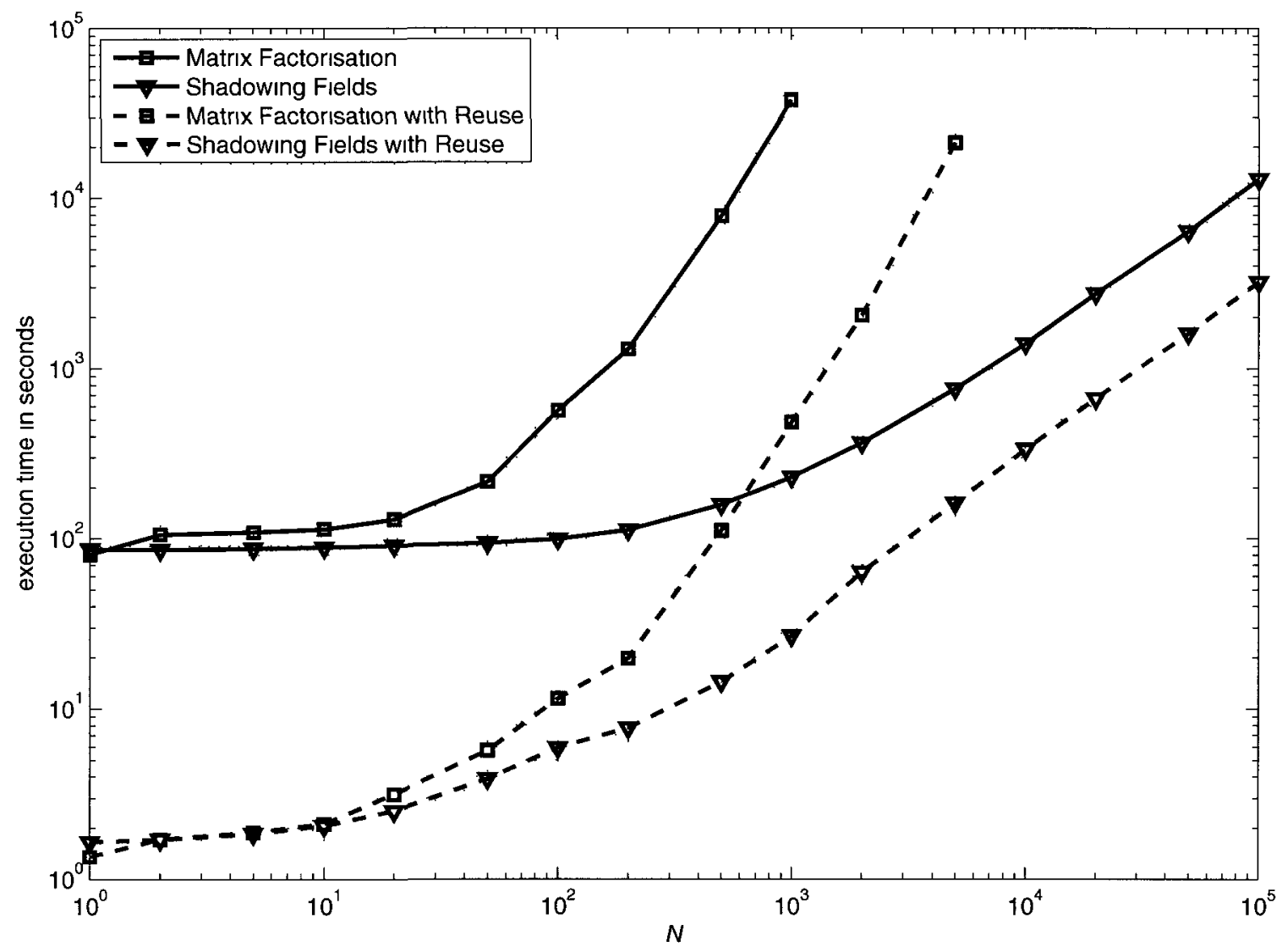

Figure 5.18: Execution time performance of matrix (Cholesky) factorisation versus shadowing fields, and of no reuse versus random sample reuse. Simulation parameters are given in Table 5.2 . 
$\mathcal{O}\left(N^{3}\right)$. That the observed growth is less than $\mathcal{O}\left(N^{3}\right)$ can be explained in part by the heavy computational cost associated with constructing the correlation matrices (cost of $\mathcal{O}\left(N^{2}\right)$ ), and in part by the fact that the correlation matrix is relatively sparse, which can reduce the computational cost.

Turning now to the use of random sample reuse, we observe the most significant gain for matrix factorisation. Indeed, while using less random values (by a factor of $K / K_{\vec{r}}=K / K_{\mathrm{Ch}}=100$ ), we observe a time gain of a factor of 78 at $N=1000$. This is because both generating the correlation matrix and factorising it, the two most expensive computations, are now done 100 fewer times, giving nearly the same gain in time. On the other hand, the shadowing field approach presents more modest gains: while for small $N$ the time gain is of a factor of 50, asymptotically it tapers off to only a factor of 4 . The bottleneck in this case is extracting the values from the shadowing field realisations based on the interferers' positions. This operation by its very nature does not benefit from reuse.

While the exact performance of each algorithm is dependent on software implementation and the hardware platform, the general trends and conclusions hold, since the results are consistent with the predicted complexity of each algorithm.

\subsubsection{Moment-Corrected Extrapolations for High $N$}

As explained in Section 4.2.3, under proper normalisation, the distribution of $I$ converges as $N$ grows. It does not matter to what exact distribution this convergence happens, only that it occurs. In fact, due to the complexity of the problem, it is not even certain that the limit distribution has any particular closed form (though we show in Section 5.2.3 the approximate convergence to a lognormal distribution in some cases).

This convergence can be exploited in accelerating simulations. Indeed, if we want the distribution of $I$ for $N M$ interferers, such that $N, N M \in \mathbb{N}^{*}, N$ is large, and 
$M>1$, we may merely simulate $I$ with $N$ interferers, and then use the knowledge of the moments of $I$ as a function of $N$ to correct the simulated distribution to match the desired one. Because of convergence, the shape (in the linear domain) of the distribution does not change when $N$ is very high; only the scale and offset parameters do.

In Section 4.2.3, we have given three formulae for extrapolating distributions. Each comes in two versions: a finite one, where the distribution for $N M$ interferers is obtained from that for $N$ interferers, and an infinite one, where the distribution for $N$ interferers is obtained from that of $I / N$ as $N \rightarrow \infty$. Because we do not have an efficient method to obtain the limiting distribution as $N \rightarrow \infty$ (though we hope that such a method will be developed in the future), we only use the first, finite, approach.

The first approach, given by (4.24), is based on simply matching the mean of $I$, which is exactly proportional to $N$. This method is the simplest, does not require the numerical computation of $A, B$, and $C$, and requires only that we multiply the simulated values of $I$ by $M$, equivalently shifting the distribution to the right by $10 \log _{10} M \mathrm{~dB}$ on lognormal paper. Figure 5.19 shows the distribution of $I$ for $N=10000$ (circles), as well as various simulations for lower $N$ and the appropriate correction factor $M=10000 / N$.

The second approach, given by (4.26), is based instead on matching only the variance of $I$. This method requires the numerical computation of $A, B$, and $C$, to obtain $c$ in (4.27). It is similar to the first approach, in that only a multiplying factor is applied to the simulated values of $I$, equivalently shifting the distribution to the right by $10 \log _{10} c \mathrm{~dB}$ on lognormal paper. Figure 5.20 is made along the same lines as Figure 5.19, but with variance-matching instead.

The third approach, given by (4.31), is based on matching both the mean and the variance of $I$. This method again requires the numerical computation of $A, B$, and $C$, to obtain $a$ and $b$ in (4.32). This last method is different from the first two in that 


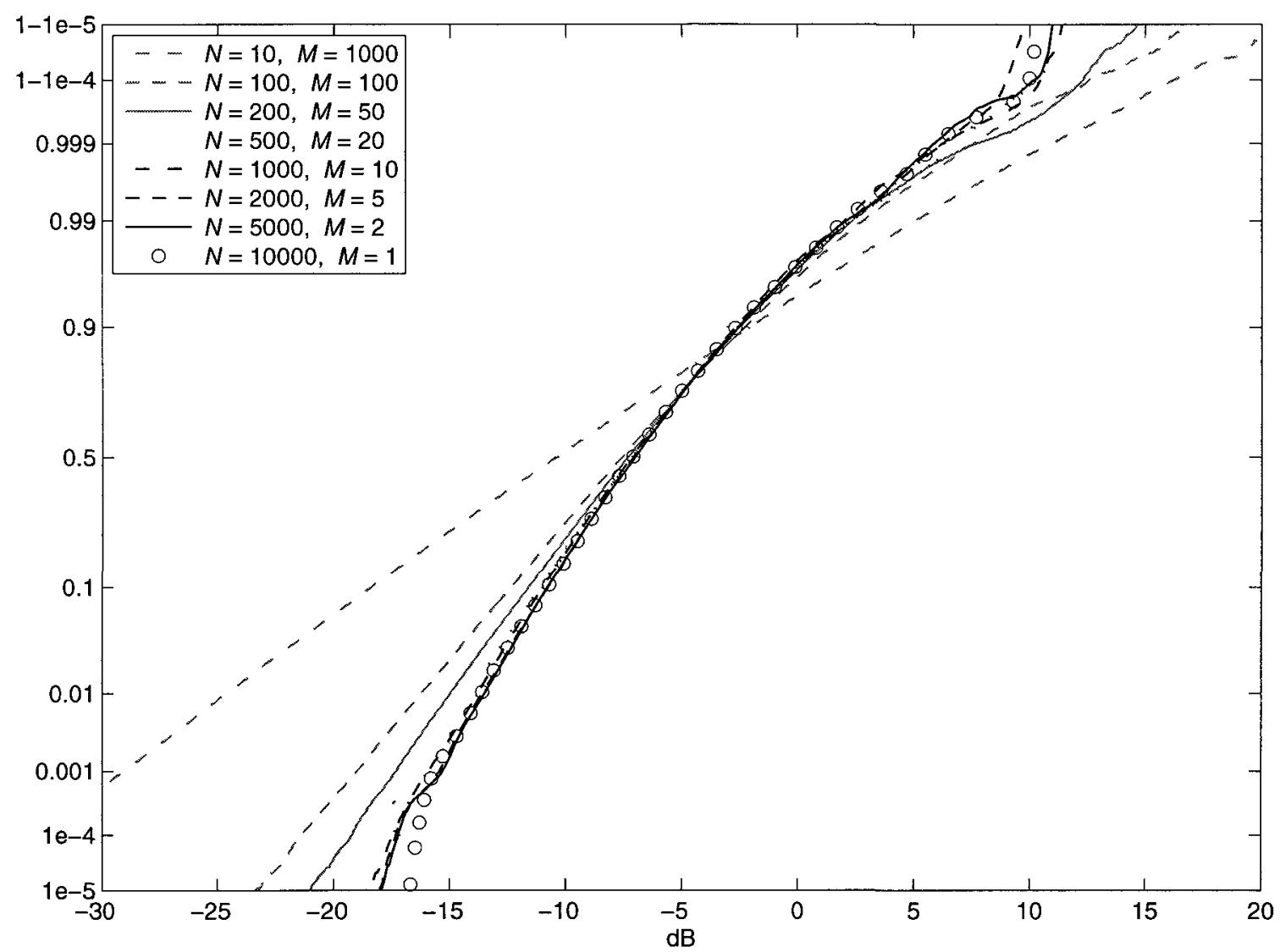

Figure 5.19: Using mean-matching (4.24) to extrapolate the cdf of $I$ for very high $N$. All simulations are done using shadowing fields with reuse. 


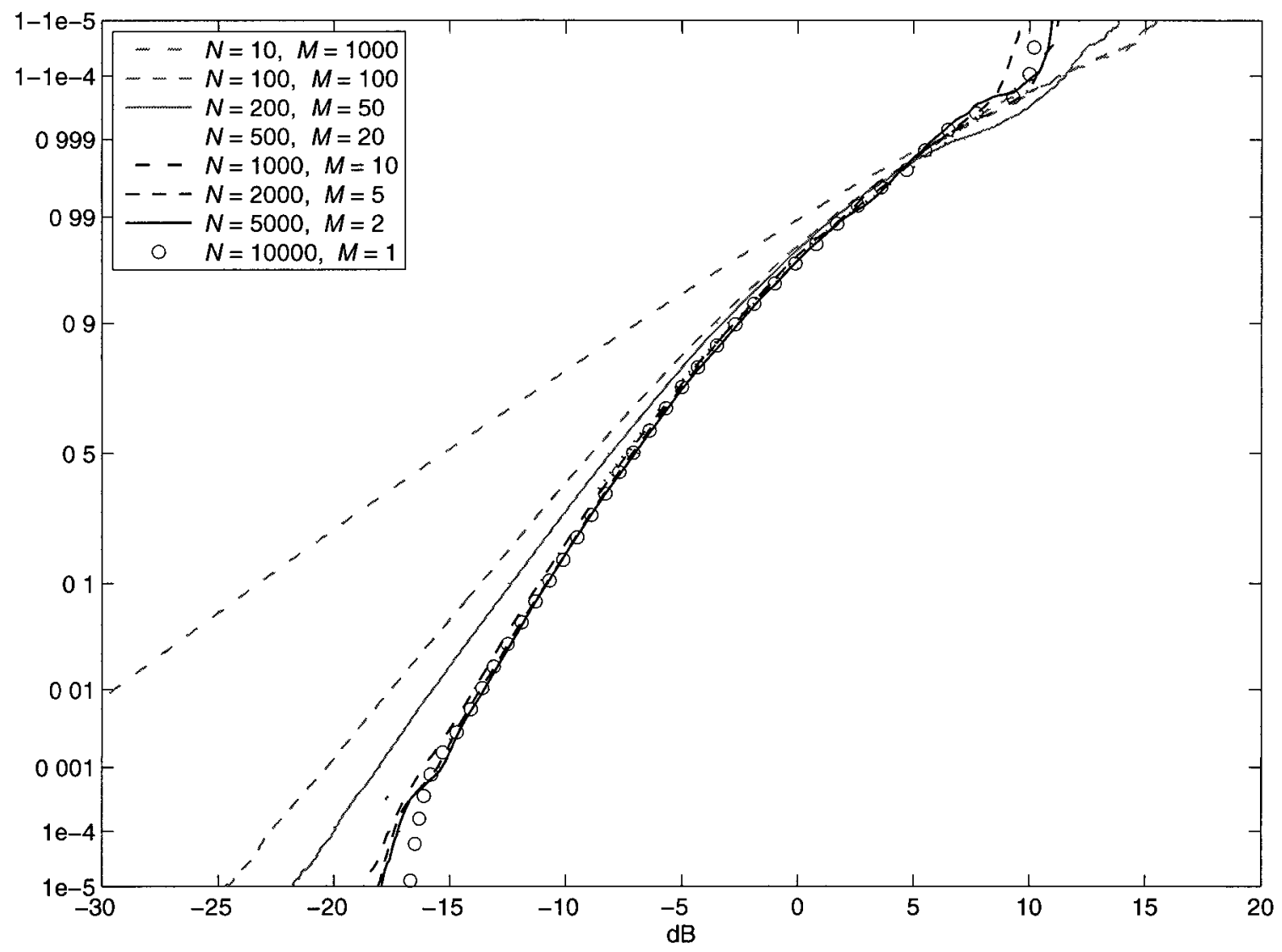

Figure 5.20: Using variance -matching (4.26) to extrapolate the cdf of $I$ for very high $N$. All simulations are done using shadowing fields with reuse. 


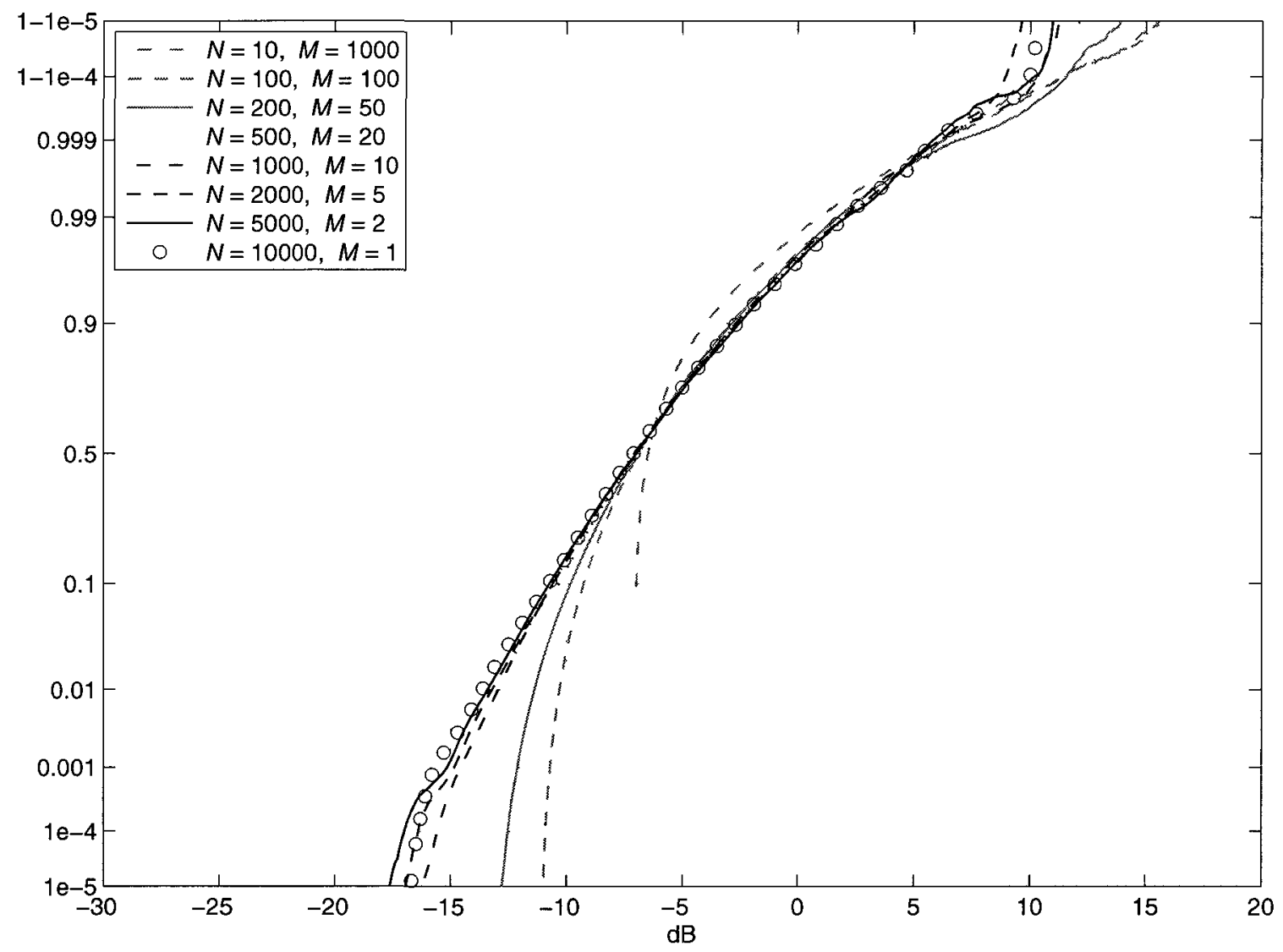

Figure 5.21: Using two-moment-matching (4.31) to extrapolate the cdf of $I$ for very high $N$. All simulations are done using shadowing fields with reuse. 
it proposes not merely a linear, but rather an affine transformation on the values of $I$, which leads to distortion, particularly in the lower tail, when plotted on lognormal paper. Figure 5.21 is made along the same lines as the previous two figures, but matching both mean and variance (equivalently, the first two moments).

The second and third methods require the numerical evaluations of the integrals in (4.20), (4.22), and (4.23). The first two integrals are one-dimensional, and are performed using basic Riemann integration with 100000 uniform points, with negligible computational time. The integral in (4.23) is four-dimensional, and is performed again using Riemann integration with $50^{4}$ uniform points, with a computational time of approximately 1.25 seconds. The values obtained for the scenario described in Table 5.2 and used in Figures 5.20 and 5.21 are:

$$
\begin{aligned}
& A=2.972 \cdot 10^{-5}, \\
& B=1.256 \cdot 10^{-7}, \\
& C=2.342 \cdot 10^{-9} .
\end{aligned}
$$

For all three methods, we observe that good convergence (within $1 \mathrm{~dB}$ ) can be obtained after about $N \geq 500$. Looking back at Figure 5.18, we observe that a simulation with $N=500$ requires about 14.4 seconds of computational time, when using both shadowing fields and random sample reuse. With the additional computational cost of $A, B$, and $C$, the distribution of $I$ for any $N$ can be well-approximated in under 16 seconds, with the help of extrapolation.

Comparing the performance of the three extrapolation methods at $N=500$, we see no particular difference in the overall accuracy (the only difference is in the far tails, where a slight accuracy trade-off can be made). We may therefore recommend mean-matching as the simplest approach, as it does not require the computation of $A, B$, and $C$. 


\subsubsection{Optimising Other Correlation Models}

While we have argued for the many advantages of the model in (3.5), many other models could be realised with shadowing fields with a little creativity, and of course reuse and extrapolation are methods that do not depend at all on the correlation model. To generate shadowing fields according to other correlation functions, one must first find a transformation (similarly as with $\mathcal{T}_{\mathrm{LP}}$ ) under which the underlying field is stationary. After that, one might exploit the particular form of the correlation (e.g., autoregressive) for an efficient implementation, or, generally, execute convolutions with the appropriate kernel using the fast Fourier transform (FFT) algorithm, as shown in [164]. The complexity of FFT is $\mathcal{O}\left(D_{\Theta} D_{\mathrm{R}} \ln D_{\Theta} D_{\mathrm{R}}\right)$ [163], not much higher than that for separable triangular correlations. Further discussions on generating stationary Gaussian fields can be found in $[60,165]$.

Furthermore, the FFT algorithm can be executed with significant time gains on specialized hardware, notably on many supported Graphics Processing Units [166], which are commercially available and already included in many current desktop computers.

Finally, an important consideration is to ensure that the correlation model used is feasible, as explained in Chapter 2. Otherwise, generating shadowing fields would be by definition impossible. 


\section{Chapter 6}

\section{Conclusion and Future Work}

In this thesis, we formulate a new interference problem that incorporates many flexible and realistic components that were only present in part in various (sometimes very different) problems studied in the literature of wireless interference. Furthermore, we demonstrate two solutions, one analytical for a limited set of layouts, the other simulation-based, that offer very simple, fast, and accurate solutions to this very involved problem.

\subsection{Achievements of this Thesis}

The principle contributions of our work are as follows:

1. A new problem formulation that allows full flexibility of the models describing the pathloss, the layout of ISs, and the shadowing spread and correlation. The incorporation of correlation in shadowing is particularly important in increasing the realism of the model. The new formulation incorporates the respective strengths of works in the areas of sums of lognormal RVs [13, 14, 17-23], in-

terference from Poisson fields [4,11,24-35], and shadowing fields [37-42], while transcending the limitations of each. 
2. A thorough literature review and comparative study of existing models for correlation in shadowing, accompanied by a formulation of mathematical and physical criteria required from a good model, and the identification of an optimal model according to these criteria.

3. A limit theorem in the field of sums of correlated lognormal RVs.

4. A very simple semi-analytical approximation in the case of large interferer clusters.

5. A highly optimised simulation algorithm that solves the problem for an arbitrarily large network in seconds, where typical simulations used to take hours.

Some interesting simple insights emerge that may be used as "rules of thumb" for further research.

1. The correlation model for shadowing given by (3.5) and based on [48] has many overwhelming advantages over many other models in existence. We therefore recommend its use to anyone working with correlation unless there are very specific reasons against it, and until research reaches a much better point of understanding of correlation in shadowing.

2. The sum of exchangeable and augmentable strictly-correlated lognormals converges to a lognormal in distribution. The convergence is more rapid for a higher correlation coefficient and for a lower spread.

3. The total interference from a large cluster of ISs may be well-approximated by a lognormal RV with easy-to- find parameters.

4. Furthermore, the above approximation does not require the knowledge of the entire shadowing correlation function, but rather only of its average value for the 
given IS layout. This quantity is much easier to obtain from field measurements than the complete correlation function.

5. An accurate cdf of the total interference from any network, no matter how large, may be computed with 16 seconds on a personal computer when making use of our algorithms.

6. Generating an equivalent stochastic process and then sampling it (as we have done with shadowing fields) may be computationally more efficient than calculating the correlation matrix of a discrete set of correlated random quantities and performing correlation matrix factorisation. This is particularly true if the correlation structure has some interesting properties.

7. When parallel sets of RVs are generated in Monte Carlo simulation, it may be possible to reduce the number of generated terms and recombine them in a way that keeps enough randomness in the simulation to obtain accurate results. The time and memory gains can be substantial.

8. When looking at large sums of similar RVs that have some structure (e.g., they are exchangeable), the distribution is expected to converge to a particular shape after a certain number of terms. It is not necessary to know the shape of this limiting distribution, but rather only to have information about the scaling of the mean and variance of the sum, so as to be able to extrapolate the sum distribution for higher numbers of terms.

9. A difficult problem might have a good solution that is a judicious hybrid of analysis, numerical techniques, and Monte Carlo simulation. The joint goal of accuracy, low computational load, and minimal human effort can thus be achieved, and this on a complex problem that models reality well. Such results are no doubt of interest to developers in industry. 
10. Exchangeability is a very strong assumption that is difficult to violate in a large network of similar devices. The results in this branch of probability, particularly in the asymptotic regime [64], should be studied carefully; they may yield very powerful results in many scientific branches that involve large numbers of similar components, where simulation becomes prohibitively expensive.

In summary, we both present a new problem formulation that is more realistic and flexible than previous approaches, and give some efficient solutions to solve it. The work does not stop there: our approach is not closed, but indeed extensible to include even more realism. We suggest further research along those lines, for which we have hopefully laid solid foundations. In the next section we show some of the most obvious model extensions, and how they can be analysed and simulated.

\subsection{Immediate Model Extensions}

Due to time limitations, we were not able to pursue certain generalisations of the model described in Chapter 3, nor perform a simulation campaign that would do them justice. We present here some quick ideas on how to extend our work in these

directions, some of which we may incorporate into [54]. We do not expect these extensions to conflict in any way; they may be added cumulatively.

\subsubsection{Random Number of Interferers}

In the same way that we have argued that in large networks IS positions would only be known statistically, it also makes sense that knowledge of $N$ is merely statistical. A Poisson distribution might be a good candidate for a model, particularly if the ISs each transmit with a low probability, and the Poisson approximation to a Binomial distribution applies. 
Incorporating a random $N$ would not be too difficult, and requires the following changes:

- To obtain the lognormal approximation for $I$ when $N$ is random, a very simple computational approach can be taken. Indeed, the expressions for $m_{I}$ and $s_{I}$ contain $N$ explicitly: we may therefore compute the approximating lognormal distribution corresponding to each possible value of $N$, and then take the weighted mixture of these lognormals, which would give the following approximation for the cdf of $I$ :

$$
\sum_{n=1}^{\max N} \mathbb{P}(N=n) \Phi\left(\frac{\ln x-m_{I} \mid N}{s_{I} \mid N}\right)
$$

where $\Phi(x)$ is the standard Gaussian cdf. The result would not necessarily be lognormal. This would be quick to compute, as $G_{n}$ and $G_{\text {cor }}$ are the only real computational cost, and they do not vary with $N$.

- The moments of $I$ would be updated from (4.19) to

$$
\begin{aligned}
\mathbb{E}\{I\} & =\mathbb{E}\{N\} A, \\
\mathbb{E}\left\{I^{2}\right\} & =\mathbb{E}\{N\} B+\left(\mathbb{E}\left\{N^{2}\right\}-\mathbb{E}\{N\}\right) C, \\
\mathbb{V A R}\{I\} & =\mathbb{E}\{N\}(B-C)+\mathbb{E}\left\{N^{2}\right\} C-\mathbb{E}^{2}\{N\} A^{2} .
\end{aligned}
$$

The appropriate substitutions then need to be made in Section 4.2.3. In the case of mean-matching, the scaling factor simply becomes $\mathbb{E}\{N\}$.

- Simulation would not be much affected: indeed, it would simply require generating a random value for $N$ before each trial of $I$. The difficulty again comes with random sample reuse, as it would require matching vectors of incompatible sizes. A possible solution would be to generate a fixed number of ISs each time that would correspond to the maximum value of $N$, then, after performing 
reuse, to reject some interferers in a random fashion so as to obtain the desired distribution for $N$. This would add only a marginal computational cost, as long as the spread of $N$ is not high.

\subsubsection{Non-Independent Interferer Positions}

It is also not too difficult to incorporate statistical dependencies between the IS positions as described, e.g., in $[151,152]$. The following considerations must be made:

- The lognormal approximation of Section 4.1.2 can still be performed in principle. The difference comes in the evaluation of the cross-moments of $\ln I_{2}$ in (4.12): while $\mathbb{E}\left\{\ln I_{\imath}^{n}\right\}$ remains the same, $\mathbb{E}\left\{\ln I_{\imath} I_{j}\right\}, i \neq j$ needs to be rewritten: the quantity $\mathbb{E}\left\{\ln r_{\imath} \ln r_{j}\right\}$ does not evaluate to $G_{1}^{2}$, but to a new geometric coefficient that must be evaluated through a four-dimensional integral. This new coefficient may be interpreted as "additional correlation" on top of the shadowing correlation contained in $G_{\text {cor }}$. The equations that follow, up to (4.16), need to be appropriately rewritten.

- The evaluation of the moments of $I$ would remain essentially the same, if we denote $g(\vec{r})$ the marginal distribution of every $\vec{r}_{i}$. What would be different is the integral evaluation of $C$ in $(4.23)$, where $g\left(\overrightarrow{r_{1}}\right) g\left(\overrightarrow{r_{2}}\right)$ would be replaced by the corresponding second-order joint distribution.

- There are no additional simulation issues in our context. It is simply necessary to replace the generation of independent $\vec{r}_{\imath}$ 's with an algorithm that generates the desired position statistics.

\subsubsection{Small-Scale Fading and Variable Interferer Transmit Power}

Let us consider both small-scale fading and random transmit power together for mathematical convenience. Indeed, since small-scale fading tends to be almost decorrelated 
at a distance of a few wavelengths, we may expect the fading on the propagation path of each IS to be roughly independent, quite contrarily to shadowing. On the other hand, we assume that all ISs transmit with iid powers, though this may not in general be true, and actually depends on the medium access and network layers. Still, a lot can be done with the iid assumption for both fading and transmit power. We can consider them jointly by considering the product of the two for each IS and calling it $T_{\imath}$. Then $\left\{T_{\imath}\right\}_{\imath=1}^{N}$ is again a set of iid RVs. The total interference then takes the form

$$
I=\sum_{\imath=1}^{N} I_{\imath}, \quad I_{\imath}=p\left(r_{\imath}\right) e^{\lambda S_{\imath}} T_{\imath}
$$

or, simplified for cluster geometries,

$$
I_{\imath}=r_{\imath}^{\beta} e^{\lambda S_{\imath}} T_{\imath}
$$

We may then make the following substitutions:

- In (4.12), we need to add the following terms

$$
\begin{array}{ll}
\text { add } \mathbb{E}\left\{\ln T_{1}\right\} & \text { to } \mathbb{E}\left\{\ln I_{\imath}\right\}, \\
\text { add } \mathbb{E}\left\{\ln ^{2} T_{1}\right\}-2 \beta G_{1} \mathbb{E}\left\{\ln T_{1}\right\} & \text { to } \mathbb{E}\left\{\ln ^{2} I_{\imath}\right\}, \\
\text { add } \mathbb{E}^{2}\left\{\ln T_{1}\right\}-2 \beta G_{1} \mathbb{E}\left\{\ln T_{1}\right\} & \text { to } \mathbb{E}\left\{\ln I_{\imath} \ln I_{\jmath}\right\},
\end{array}
$$

and make the corresponding substitutions in (4.14)-(4.16).

- In (4.19)

$$
\begin{aligned}
& A \rightarrow A \mathbb{E}\left\{T_{1}\right\}, \\
& B \rightarrow B \mathbb{E}\left\{T_{1}^{2}\right\}, \\
& C \rightarrow C \mathbb{E}^{2}\left\{T_{1}\right\}
\end{aligned}
$$


and applying them to all equations in Section 4.2.3. These updated equations should then be used in simulation extrapolations in Section 5.3.6, though there is in fact no change for the mean-matching method, which does not depend on $A, B$, or $C$.

- In (4.36), the result would simply be multiplied by $\mathbb{E}\left\{T_{1}\right\}$, which is verified in [51] and is a consequence of the LLN.

- The simulation algorithms do not change much: all that is needed is to generate iid trials of $T_{2}$, and then incorporate them into $I[k]$. The only difficulty is how to do random sample reuse: indeed we now have not two, but potentially four (if one separates fading and transmit power) random quantities for each IS, which can be separated or coupled in many different combinations. It is not evident which is the best way to do this, and several ideas should be tried.

\subsubsection{Directional Victim Receiver Antenna}

Our work also extends easily to consider any azimuth angle gain pattern of the RX receive antenna. We call $G(\angle \vec{r})$ the antenna power gain [167], and therefore each $I_{\imath}$ is multiplied by $G\left(\angle \vec{r}_{2}\right)$.

The necessary changes to our work are then as follows:

- It is necessary to replace $g(\vec{r})$ with $g(\vec{r}) G(\angle \vec{r})$ everywhere in Section 4.3.

- The analysis in Section 4.1.2, which has been shown to give a good approximation for cluster geometries, is also of interest in the case of a highly-directive RX antenna. Indeed, assume that the ISs are not distributed according to a cluster geometry, but are located all around the RX (e.g., layout C in Figure 3.1); and assume also that the $\mathrm{RX}$ receive antenna is directional, with a high gain from a relatively narrow angular region. We could then say that most of the effective 
interference power is coming from that narrow angular region, which is similar to the cluster geometry case.

In order to update the analysis, we would replace $g(\vec{r})$ with $g(\vec{r}) \bar{G}(\angle \vec{r})$ in Section 4.1 .2 , where $\bar{G}(\angle \vec{r})=k_{G} \cdot G(\angle \vec{r})$ such that $\int_{\mathcal{A}_{g}} g(\vec{r}) \bar{G}(\angle \vec{r}) d \vec{r}=1$, and also multiply the total interference power by $k_{G}$. The integrals (4.17) for $G_{n}$ and $G_{\text {cor }}$ would need to be updated accordingly. This substitution can be justifiable for high $N$, given that the integral approximation in Section 4.3 is then appropriate, where we have made the same substitution. Thus a higher antenna power gain has approximately the same effect as a proportional increase in the density of interferers.

We would then expect the new average correlation coefficient $G_{\text {cor }}$ to be much higher, and the approximations given in Section 4.1.2 to be accurate.

- The quantities $A, B$, and $C$ would be updated from (4.18) to

$$
\begin{aligned}
& A=\mathbb{E}\left\{I_{1}\right\}=\mathbb{E}\left\{p\left(r_{1}\right) e^{\lambda S_{1}} G\left(\angle \vec{r}_{1}\right)\right\}, \\
& B=\mathbb{E}\left\{I_{1}^{2}\right\}=\mathbb{E}\left\{p^{2}\left(r_{1}\right) e^{2 \lambda S_{1}} G^{2}\left(\angle \vec{r}_{1}\right)\right\}, \\
& C=\mathbb{E}\left\{I_{1} I_{2}\right\}=\mathbb{E}\left\{p\left(r_{1}\right) p\left(r_{2}\right) e^{\lambda\left(S_{1}+S_{2}\right)} G\left(\angle \vec{r}_{1}\right) G\left(\angle \vec{r}_{2}\right)\right\}
\end{aligned}
$$

and the integrals in (4.20), (4.22), and (4.23) would be updated accordingly.

- The simulation algorithms would not change in principle. Simply, each $I_{i}$ would be multiplied by $G\left(\angle \vec{r}_{i}\right)$ before the summation.

\subsubsection{Other Dimensions: Time and Frequency}

The work in this thesis presents what might be called a "snapshot analysis", i.e., it studies the distribution of $I$ at any given time. This, however, tells us little about how $I$ might evolve in time, due to RX or IS mobility, and possibly due to the changing 
channel. Similarly, the analysis is single-frequency: it would be interesting to know how the interference correlates across frequencies, e.g., in frequency-division multiplexing systems. To incorporate these two additional dimensions, one would have to start by finding good models for shadowing correlation in time and in frequency.

\subsection{Long-Term Research Questions}

Our work in wireless interference and propagation, and in the applied mathematical challenges therein, has inspired us to think about larger problems in this field. We hope to pursue some of these topics in our future research career.

- As we mentioned before, the theory of exchangeable RVs may be a very powerful tool with wide applications to problems where there are many similar components and simulation is prohibitive. We hope to study this mathematical theory further, as we believe it may have a wide range of applications across the physical sciences.

- Consider the joint distribution of two or more shadowing paths originating at the same point. Field measurements can give us an idea of the correlation coefficient among them. In simulations it is further assumed that the joint distribution of these paths is jointly lognormal (that is, their logarithms form a Gaussian vector). This is a useful assumption for simulations, since the correlation matrix can specify the entire joint distribution, and there is an easy construction of the Gaussian vector. However, there is probably no physical or empirical evidence for the jointness of shadowing paths. It would be interesting to study, via either measurements or a powerful theoretical model, what the joint distribution of shadowing paths is and whether it can be well-approximated by a jointly Gaussian structure (copula [17]). Some of our results indicate that two joint distributions with the same marginal cdfs and the same correlation matrix may 
nevertheless give rise to very different total interference statistics. Of course, this presents a great analytical challenge.

- Also, can we truly say that shadowing is exactly lognormal? Some counterarguments come to mind, particularly surrounding the shape of the tails. In particular, the lognormal RV takes values on $[0,+\infty)$, which suggests that a receiver might actually receive an arbitrarily high interference power from a finite-power transmitter, albeit very rarely. It would be well to study whether this physical contradiction is statistically significant, effectively biasing analytical results, or if it is benign. Also, can a better cdf be designed for shadowing that does not have this problem, while still being faithful to empirical data? The truncated lognormal model $[10,144]$ attempts to solve this problem, but its sharp transition at the truncation point does not seem plausible in a physical phenomenon.

- Additionally, why does shadowing appear to be lognormal? By "why" we mean, how can it be explained from basic well-established principles of wave propagation physics? The traditional explanation has usually been based on a multiplicative CLT argument. However, a deeper reflection shows that the proposed explanation is physically unconvincing, and may not correspond to measurements. Notably, measurements show that the shadowing $\mathrm{dB}$ spread typically does not vary (much) with distance, which is not what the traditional argument would predict. Some researchers, notably Jari Salo [168], are currently working on better explanations for the lognormality of shadowing, which might lead us to a deeper understanding of this phenomenon, and perhaps a better propagation model.

- It would be valuable also to examine the literature on field measurements pertaining to average path loss, and especially shadowing, in order to have a better 
understanding of how the mathematical models came about, and whether there is any more information to be extracted from this data. For example, how constant is the shadowing $\mathrm{dB}$ spread versus distance? This would be helpful to get a deeper theoretical understanding of these propagation phenomena.

It is our intuition that the above problems could be examined by some kind of ray-tracing arguments, as [168] suggests. Furthermore, ray-tracing approaches may be becoming a viable alternative to field measurements, for two reasons:

1. Several indoor and outdoor ray-tracing simulations have demonstrated excellent predictions of real received power measurements [169-172].

2. The cost of the heavy computations required is falling rapidly, while the cost of doing measurements in the field follows no such law.

We have demonstrated in this thesis that correlated shadowing makes a big difference in interference analysis, at least for large networks. If it is true that ray-tracing can predict reality well, it would be most interesting to see if we can bridge a statistical wireless model that includes correlated shadowing with a ray-tracing model. We would then be able to evaluate how close our work corresponds to reality. The difficulty is that ray-tracing is mostly deterministic, and based on ray propagation in a fixed environment, whereas shadowing and fading assume statistically distributed obstacles. A first step in bridging the two approaches would then require randomising the environment in which ray-tracing is performed, and extracting some useful statistics therefrom. 


\section{Bibliography}

[1] K. David, Ed., Technologies for the Wireless Future: Wireless World Research Forum. Wiley, Oct. 2008, vol. 3.

[2] S. R. Hall, A. W. Jeffries, S. E. Avis, and D. D. N. Bevan, "Performance of open access femtocells in 4G macrocellular networks," WWRF Meeting 20, Tech. Rep., Apr. 2008.

[3] R. Hekmat and P. V. Mieghem, "Interference power sum with log-normal components in ad-hoc and sensor networks," in Proc. International Symposium on Modelıng and Optımızatıon in Mobıle, Ad Hoc, and Wireless Networks, Apr. 2005.

[4] M. Win, P. Pinto, and L. Shepp, "A mathematical theory of network interference and its applications," Proc. IEEE, vol. 97, pp. 205-230, Feb. 2009.

[5] M. Salem, A. Adinoyi, and H. Yanikomeroglu, "Integrating self-organizing nomadic relays into OFDMA fixed-relay cellular networks," under review in IEEE Trans. Mobıle Comput., Oct. 2010.

[6] M. H. Ismail and M. M. Matalgah, "Outage probability analysis in cellular systems with noisy Weibull-faded lognormal-shadowed links," in Proc. IEEE Symposıum on Computers and Communıcatıons, June 2005, pp. 269-274. 
[7] F. Berggren, "An error bound for moment matching methods of lognormal sum distributions," Eur. Trans. Telecommun., vol. 16, pp. 573-577, Mar. 2005.

[8] W. Mohr, "Heterogeneous networks to support user needs with major challenges for new wideband access systems," Wireless Pers. Commun., vol. 22, pp. 109137, Aug. 2002.

[9] B. Hagerman, "Downlink relative co-channel interference powers in cellular radio systems," in Proc. IEEE Vehicular Technology Conference, vol. 1, July 1995, pp. 366-370.

[10] J. Salo, L. Vuokko, H. M. El-Sallabi, and P. Vainikainen, "An additive model as a physical basis for shadow fading," IEEE Trans. Veh. Technol., vol. 56, pp. 13-26, Jan. 2007.

[11] M. Aljuaid and H. Yanikomeroglu, "Investigating the Gaussian convergence of the distribution of the aggregate interference power in large wireless networks," IEEE Trans. Veh. Technol., vol. 59, no. 9, pp. 4418-4424, Nov. 2010.

[12] IEEE Commun. Mag., Jan. 2011, Network Testing Series.

[13] L. Fenton, "The sum of log-normal probability distributions in scatter transmission systems," IEEE Trans. Commun., vol. 8, pp. 57-67, Mar. 1960.

[14] S. S. Szyszkowicz, "Interference in cellular networks: Sum of lognormals modeling," Master's thesis, Carleton University, Jan. 2007, can be obtained at www.sce.carleton.ca/ ${ }^{\sim}$ sz/SzyszkowiczMASc.pdf.

[15] S. S. Szyszkowicz and H. Yanikomeroglu, "On the tails of the distribution of the sum of lognormals," in Proc. IEEE International Conference on Communications, June 2007, pp. 5324-5329. 
[16] _ _ - "Fitting the modified-power-lognormal to the sum of independent lognormals distribution," in Proc. IEEE Global Telecommunications Conference, Nov.-Dec. 2009, p. 6.

[17] S. Asmussen and L. Rojas-Nandayapa, "Asymptotics of sums of lognormal random variables with Gaussian copula," Stat. Probabil. Lett., vol. 78, pp. 2709 2714, Nov. 2008.

[18] M. Di Renzo, F. Graziosi, and F. Santucci, "Cooperative spectrum sensing in cognitive radio networks over correlated log-normal shadowing," in Proc. IEEE Vehicular Technology Conference, Apr. 2009.

[19] M. Di Renzo, F. Graziosi, and F. Santucci, "Further results on the approximation of log-normal power sum via Pearson type IV distribution: a general formula for log-moments computation," IEEE Trans. Commun., vol. 57, no. 4, pp. 893-898, Apr. 2009.

[20] M. Di Renzo, L. Imbriglio, F. Graziosi, and F. Santucci, "Smolyak's algorithm: A simple and accurate framework for the analysis of correlated log-normal power-sums," IEEE Commun. Lett., vol. 13, no. 9, pp. 673-675, Sept. 2009.

[21] X. Gao, H. Xu, and D. Ye, "Asymptotic behavior of tail density for sum of correlated lognormal variables," Internat. J. Math. Math. Sci., pp. 1-28, 2009.

[22] C. Tellambura and D. Senaratne, "Accurate computation of the MGF of the lognormal distribution and its application to sum of lognormals," IEEE Trans. Commun., vol. 58, no. 5, pp. 1568-1577, May 2010.

[23] A. S. H. Mahmoud, "New quadrature-based approximations for the characteristic function and the distribution function of sums of lognormal random variables," to appear in IEEE Trans. Veh. Technol., p. 10, Dec. 2010. 
[24] E. S. Sousa and J. A. Silvester, "Optimum transmission ranges in a direct-sequence spread-spectrum multihop packet radio network," IEEE J. Sel. Areas Commun., vol. 8, no. 5, pp. 762-771, June 1990.

[25] E. Sousa, "Performance of a spread spectrum packet radio network link in a Poisson field of interferers," IEEE Trans. Inf. Theory, vol. 38, no. 6, pp. 1743 1754, Nov. 1992.

[26] J. Ilow, D. Hatzinakos, and A. Venetsanopoulos, "Performance of FH SS radio networks with interference modeled as a mixture of Gaussian and alpha-stable noise," IEEE Trans. Commun., vol. 46, no. 4, pp. 509-520, Apr. 1998.

[27] J. Ilow and D. Hatzinakos, "Analytic alpha-stable noise modeling in a Poisson field of interferers or scatterers," IEEE Trans. Signal Process., vol. 46, no. 6, pp. 1601-1611, June 1998.

[28] X. Yang and A. P. Petropulu, "Co-channel interference modeling and analysis in a Poisson field of interferers in wireless communications," IEEE Trans. Signal Process., vol. 51, no. 1, pp. 64-76, Jan. 2003.

[29] R. H. Etkin, "Spectrum sharing: Fundamental limits, scaling laws, and selfenforcing protocols," Ph.D. dissertation, University of California, Berkeley, 2006.

[30] A. Hasan and J. G. Andrews, "The guard zone in wireless ad hoc networks," IEEE Trans. Wireless Commun., vol. 6, no. 3, pp. 897-906, Mar. 2007.

[31] A. Ghasemi and E. Sousa, "Interference aggregation in spectrum-sensing cognitive wireless networks," IEEE J. Sel. Topıcs Signal Process., vol. 2, pp. 41-56, Feb. 2008. 
[32] E. Salbaroli and A. Zanella, "Interference analysis in a Poisson field of nodes of finite area," IEEE Trans. Veh. Technol., vol. 58, no. 4, pp. 1776-1783, May 2009.

[33] P. Pinto, A. Giorgetti, M. Z. Win, and M. Chiani, "A stochastic geometry approach to coexistence in heterogeneous wireless networks," IEEE J. Sel. Areas Commun., vol. 27, no. 7, pp. 1268-1282, Sept. 2009.

[34] K. Gulati, B. L. Evans, J. G. Andrews, and K. R. Tinsley, "Statistics of cochannel interference in a field of Poisson and Poisson-Poisson clustered interferers," IEEE Trans. Signal Process., vol. 58, no. 12, pp. 6207-6222, Dec. 2010.

[35] M. Aljuaid and H. Yanikomeroglu, "Impact of secondary users field size on spectrum sharing opportunities," in Proc. IEEE Wireless Communicatıons and Networking Conference, Apr. 2010, pp. 1-6.

[36] M. Haenggi and R. K. Ganti, "Interference in large wireless networks," Foundatıons and Trends in Networkıng, vol. 3, no. 2, pp. 127-248, Feb. 2009.

[37] K. Kumaran, S. E. Golowich, and S. Borst, "Correlated shadow-fading in wireless networks and its effect on call dropping," Wirel. Netw., vol. 8, pp. 61-71, Jan. 2002.

[38] I. Forkel, M. Schinnenburg, and M. Ang, "Generation of two-dimensional correlated shadowing for mobile radio network simulation," in Proc. International Symposıum on Wireless Personal Multımedıa Communıcatıons, vol. 2, Sept. 2004, pp. 314-319.

[39] R. Fraile, J. Nasreddine, N. Cardona, and X. Lagrange, "Multiple diffraction shadowing simulation model," IEEE Commun. Lett., vol. 11, pp. 319-321, Apr. 2007. 
[40] Z. Wang, E. Tameh, and A. Nix, "Joint shadowing process in urban peer-topeer radio channels," IEEE Trans. Veh. Technol., vol. 57, pp. 52-64, Jan. 2008.

[41] R. Fraile, J. F. Monserrat, J. Gozalvez, and N. Cardona, "Mobile radio bidimensional large-scale fading modelling with site-to-site cross-correlation," Eur. Trans. Telecommun., vol. 19, pp. 101-106, Jan. 2008.

[42] D. Catrein and R. Mathar, "Gaussian random fields as a model for spatially correlated log-normal fading," in Proc. Australasıan Telecommunicatıon Networks and Applications Conference, Dec. 2008, pp. 153-157.

[43] M. Gudmundson, "Correlation model for shadow fading in mobile radio systems," IEE Electron. Lett., vol. 27, no. 23, pp. 2145-2146, Nov. 1991.

[44] P. Agrawal and N. Patwari, "Correlated link shadow fading in multi-hop wireless networks," IEEE Trans. Wireless Commun., vol. 8, no. 8, pp. 4024-4036, Aug. 2009.

[45] N. Patwari and P. Agrawal, "Effects of correlated shadowing: Connectivity, localization, and RF tomography," in Proc. Internatıonal Conference on Informatıon Processing in Sensor Networks, Apr. 2008, pp. 82-93.

[46] B. Kasiri, M. Naderi, and B. Abolhassani, "A new realistic relay selection method based on correlated shadowing for multihop cellular networks," in Proc. IEEE Internatıonal Conference on Computer and Electrical Engineering, Dec. 2008, pp. 669-673.

[47] — , "The effect of correlated link shadowing on the performance of multihop cellular networks," in Proc. IEEE Internatıonal Conference on Computer and Electrical Engineering, Dec. 2008, pp. 951-955. 
[48] T. Klingenbrunn and P. Mogensen, "Modelling cross-correlated shadowing in network simulations," in Proc. IEEE Vehıcular Technology Conference, vol. 3, Sept. 1999, pp. 1407-1411.

[49] S. S. Szyszkowicz, H. Yanikomeroglu, and J. S. Thompson, "On the feasibility of wireless shadowing correlation models," IEEE Trans. Veh. Technol., vol. 59, no. 9, pp. 4222-4236, Nov. 2010.

[50] S. S. Szyszkowicz, F. Alaca, H. Yanikomeroglu, and J. S. Thompson, "Efficient simulation using shadowing fields of many wireless interferers with correlated shadowing," in Proc. IEEE Vehıcular Technology Conference, May 2010.

[51] — "Aggregate interference distribution from large wireless networks with correlated shadowing: Approaches to simulation and analysis," submitted to IEEE Trans. Veh. Technol., pp. 1--35, Sept. 2010, manuscript VT-2010-01105.

[52] S. S. Szyszkowicz and H. Yanikomeroglu, "Limit theorem on the sum of identically distributed equally and positively correlated joint lognormals," IEEE Trans. Commun., vol. 57, no. 12, pp. 3538-3542, Dec. 2009.

[53] — " Analysis of interference from large clusters as modeled by the sum of many correlated lognormals," in Proc. IEEE Wireless Communıcatıons and Networkıng Conference, Mar.-Apr. 2008, pp. 741-745.

[54] — "A simple approximation to the aggregate interference from large interferer clusters with correlated shadowing," in preparatıon, 2010.

[55] D. S. Bernstein, Matrıx Mathematıcs: Theory, Facts, and Formulas with Applıcatıon to Lınear Systems Theory. Princeton, NJ: Princeton University Press, 2005. 
[56] A. M. Yaglom, Correlation Theory Of Stationary And Related Random Functions, Volume 1: Basic Results. Springer-Verlag, 1987.

[57] D. Avidor and S. Mukherjee, "Hidden issues in the simulation of fixed wireless systems," Wirel. Netw., vol. 7, pp. 187-200, 2001.

[58] A. Leon-Gracia, Probability and Random Processes for Electrical Engineers, 2nd ed. Addison Wesley, 1994.

[59] A. V. Vecchia, "A general class of models for stationary two-dimensional random processes," Biometrica, vol. 72, pp. 281-291, 1985.

[60] G. Storvik, A. Frigessi, and D. Hirst, "Stationary space-time Gaussian fields and their time autoregressive representation," Statistical Modelling, vol. 2, pp. 139-161, 2002.

[61] V. K. Rohatgi and A. K. M. E. Saleh, An Introduction to Probability and Statistics, 2nd ed. Wiley-Interscience, 2001.

[62] B. de Finetti, "La prévision : ses lois logiques, ses sources subjectives," Annales de l'institut Henri Poincaré, vol. 7, no. 1, pp. 1-68, 1937.

[63] — Theory of Probability: A Critical Introductory Treatment. Wiley, 1970.

[64] R. L. Taylor, P. Z. Daffer, and R. F. Patterson, Limit theorems for sums of exchangeable random variables. Rowman \& Allanheld, Publishers, 1985.

[65] E. Hewitt and L. J. Savage, "Symmetric measures on Cartesian products," Trans. Am. Math. Soc., vol. 80, pp. 470-501, Nov. 1955.

[66] D. Kitchener, M. Naden, W. Tong, P. Zhu, G. Senarnath, H. Zhang, D. Steer, and D. Yu, "Correlated lognormal shadowing model," IEEE 802.16 Session 44, Tech. Rep., July 2006. 
[67] F. Fabbri and R. Verdone, "The impact of correlated channel fluctuations on the connectivity of wireless ad-hoc networks," in Proc. IEEE Vehicular Technology Conference, Apr. 2009.

[68] J. H. Lee and R. M. Buehrer, "Location estimation using differential RSS with spatially correlated shadowing," in Proc. IEEE Global Telecommunications Conference, Nov. 2009, pp. 1-6.

[69] F. Graziosi, M. Pratesi, M. Ruggieri, and F. Santucci, "A multicell model of handover initiation in mobile cellular networks," IEEE Trans. Veh. Technol., vol. 48, no. 3, pp. 802-814, May 1999.

[70] X. Yang, S. Ghaheri-Niri, and R. Tafazolli, "Downlink soft handover gain in CDMA cellular network with cross-correlated shadowing," in Proc. IEEE Vehicular Technology Conference, vol. 1, Oct. 2001, pp. 276-280.

[71] F. Graziosi and F. Santucci, "A general correlation model for shadow fading in mobile radio systems," IEEE Commun. Lett., vol. 6, pp. 102-104, Mar. 2002.

[72] J. Kim and G. Stuber, "CDMA soft handoff analysis in the presence of power control error and shadowing correlation," IEEE Trans. Wireless Commun., pp. 245-255, Apr. 2002.

[73] L.-C. Wang, G. Stuber, and C.-T. Lea, "Effects of Rician fading and branch correlation on a local-mean-based macrodiversity cellular system," IEEE Trans. Veh. Technol., vol. 48, no. 2, pp. 429-436, Mar. 1999.

[74] J. Zhang and V. Aalo, "Effect of macrodiversity on average-error probabilities in a Rician fading channel with correlated lognormal shadowing," IEEE Trans. Commun., vol. 49, no. 1, pp. 14-18, Jan. 2001. 
[75] M. DeFaria and E. Sousa, "Effect of intercell interference on the SNIR of a multihop cellular network," in Proc. IEEE Vehıcular Technology Conference, vol. 5, May-June 2005, pp. 3107-3111.

[76] N. Gogate, D. Avidor, and S. Panwar, "Simulation study of a fixed wireless access system," in Proc. IEEE International Conference on Universal Personal Communıcatıons, vol. 1, Oct. 1998, pp. 209-215.

[77] H. Haas, S. McLaughlin, and G. Povey, "An investigation on capacity versus guard-bands in the TDD mode of UMTS," in Proc. IEEE Vehicular Technology Conference, vol. 4, Sept. 2000, pp. 1820-1824.

[78] D. Avidor, N. Hegde, and S. Mukherjee, "On the impact of the soft handoff threshold and the maximum size of the active group on resource allocation and outage probability in the UMTS system," IEEE Trans. Wireless Commun., vol. 3, no. 2, pp. 565-577, Mar. 2004.

[79] H. Arnold, D. Cox, and R. Murray, "Macroscopic diversity performance measured in the $800-\mathrm{MHz}$ portable radio communications environment," IEEE Trans. Antennas Propag., vol. 36, no. 2, pp. 277-281, Feb. 1988.

[80] S. Mukherjee and D. Avidor, "Effect of macrodiversity and microdiversity on outages and bit error rates in a cellular system," in Proc. IEEE Wireless Communicatıons and Networking Conference, vol. 2, Sept. 2000, pp. 649-654.

[81] W. Hwang, H. Kang, Y. Choi, and K. Kim, "Effects of correlated shadowing and multipath fading on OFDM systems with postdetection-combining-based macrodiversity," in Proc. International Symposium on Wireless Personal Multımedıa Communıcations, vol. 2, Oct. 2002, pp. 449-453. 
[82] S. Mukherjee and D. Avidor, "Effect of microdiversity and correlated macrodiversity on outages in a cellular system," IEEE Trans. Wireless Commun., vol. 2, no. 1, pp. 50-58, Jan. 2003.

[83] Y. Chen, L. Hu, C. Yuen, Y. Zhang, Z. Zhang, and P. Rapajic, "Intrinsic measure of diversity gains in generalised distributed antenna systems with cooperative users," IET Commun., vol. 3, pp. 209-222, Feb. 2009.

[84] C.-X. Wang, X. Hong, X. Ge, X. Cheng, G. Zhang, and J. Thompson, "Cooperative MIMO channel models: A survey," IEEE Commun. Mag., vol. 48, pp. 80-87, Feb. 2010.

[85] V. Graziano, "Propagation correlations at $900 \mathrm{MHz}$," IEEE Trans. Veh. Technol., vol. 27, no. 4, pp. 182-189, Nov. 1978.

[86] M. J. Marsan, G. C. Hess, and S. S. Gilbert, "Shadowing variability in an urban land mobile environment at $900 \mathrm{MHz}$," Electron. Lett., vol. 26, pp. 646-648, May 1990.

[87] J. Liberti and T. Rappaport, "Statistics of shadowing in indoor radio channels at 900 and $1900 \mathrm{MHz}$," in Proc. IEEE Milıtary Communzcatıons Conference, vol. 3, Oct. 1992, pp. 1066-1070.

[88] A. Mawira, "Models for the spatial correlation functions of the ( $\log )$-normal component of the variability of VHF/UHF field strength in urban environment," in Proc. IEEE Internatıonal Symposıum on Personal, Indoor and Mobıle Radio Communıcatıons, Oct. 1992, pp. 436-440.

[89] R. Stevens and I. Dilworth, "Mobile radio shadowing loss variability and cochannel signal correlation at $452 \mathrm{MHz}, "$ Electron. Lett., vol. 32, no. 1, pp. 16-17, Jan. 1996. 
[90] K. Butterworth, K. Sowerby, and A. Williamson, "Correlated shadowing in an in-building propagation environment," Electron. Lett., vol. 33, no. 5, pp. 420-422, Feb. 1997.

[91] P. Taaghol and R. Tafazolli, "Correlation model for shadow fading in landmobile satellite systems," Electron. Lett., vol. 33, no. 15, pp. 1287-1289, July 1997.

[92] T. Sorensen, "Correlation model for slow fading in a small urban macro cell," in Proc. IEEE International Symposium on Personal, Indoor and Mobıle Radio Communicatıons, vol. 3, Sept. 1998, pp. 1161-1165.

[93] K. Zayana and B. Guisnet, "Measurements and modelisation of shadowing cross-correlations between two base-stations," in Proc. IEEE International Conference on Universal Personal Communicatıons, vol. 1, Oct. 1998, pp. 101105.

[94] T. Sorensen, "Slow fading cross-correlation against azimuth separation of base stations," Electron. Lett., vol. 35, pp. 127-129, Jan. 1999.

[95] E. Perahia and D. Cox, "Shadow fading correlation between uplink and downlink," in Proc. IEEE Vehıcular Technology Conference, vol. 1, May 2001, pp. 308-312.

[96] E. Perahia, D. Cox, and S. Ho, "Shadow fading cross correlation between basestations," in Proc. IEEE Vehıcular Technology Conference, vol. 1, May 2001, pp. 313-317.

[97] J. Weitzen and T. Lowe, "Measurement of angular and distance correlation properties of $\log -$ normal shadowing at $1900 \mathrm{MHz}$ and its application to design 
of PCS systems," IEEE Trans. Veh. Technol., vol. 51, no. 2, pp. 265-273, Mar. 2002.

[98] N. Jalden, P. Zetterberg, L. Garcia, and B. Ottersten, "Inter and intra site correlation of large scale parameters from macro cellular measurements at 1800 MHz," EURASIP J. Wireless Communicatıons and Networkıng, July 2007.

[99] N. Jalden, P. Zetterberg, and B. Ottersten, "Directional dependence of large scale parameters in wireless channel models," in Proc. IEEE Wireless Communications and Networking Conference, Mar.-Apr. 2008, pp. 1223-1228.

[100] P. Dassanayake, "Spatial correlation of shadow fading and its impact on handover algorithm parameter settings," in Proc. IEEE Singapore International Conference on Networks, July 1995, pp. 225-229.

[101] G. Malmgren, "On the performance of single frequency networks in correlated shadow fading," IEEE Trans. Broadcast., vol. 43, no. 2, pp. 155-165, June 1997.

[102] J. F. Monserrat, R. Fraile, D. Calabuig, and N. Cardona, "Complete shadowing modeling and its effect on system level performance evaluation," in Proc. IEEE Vehicular Technology Conference, May 2008, pp. 294-298.

[103] R.-A. Pitaval, "Relay deployment in single frequency network," Master's thesis, Helsinki University of Technology, Aug. 2009.

[104] D. Kaltakis, M. A. Imran, and R. Hoshyar, "Uplink capacity with correlated lognormal shadow fading," in Proc. IEEE Vehıcular Technology Conference, Apr. 2009.

[105] W. Lee and D.-H. Cho, "A new neighbor discovery scheme based on spatial correlation of wireless channel," in Proc. IEEE Vehıcular Technology Conference, Apr. 2009. 
[106] A. Sibille, "Efficient generation of spatially and frequency correlated random values for cognitive radio network simulators," IEEE Trans. Veh. Technol., vol. 59, pp. 1121-1128, Mar. 2010.

[107] D. Kotz, C. Newport, and C. Elliott, "The mistaken axioms of wireless-network research," Dept. of Computer Science, Dartmouth College, Tech. Rep., July 2003.

[108] K.-J. Yang and Y.-R. Tsai, "Location tracking in mobile networks under correlated shadowing effects," in Proc. IEEE Wireless Communıcatıons and Networking Conference, Apr. 2009.

[109] A. Ghasemi and E. Sousa, "Asymptotic performance of collaborative spectrum sensing under correlated log-normal shadowing," IEEE Commun. Lett., vol. 11, no. 1, pp. 34-36, Jan. 2007.

[110] A. Ghasemi and E. S. Sousa, "Fundamental limits of spectrum-sharing in fading environments," IEEE Trans. Wireless Commun., vol. 6, no. 2, pp. 649-658, Feb. 2007.

[111] M. Di Renzo, L. Imbriglio, F. Graziosi, and F. Santucci, "Cooperative spectrum sensing over correlated log-normal sensing and reporting channels," in Proc. IEEE Global Telecommunications Conference, Nov. 2009, pp. 1-8.

[112] F. Graziosi, M. Pratesi, M. Ruggieri, and F. Santucci, "Modeling of handover initiation algorithms with correlated co-channel interferers," in Proc. IEEE International Conference on Universal Personal Communicatıons, Oct. 1997, pp. 244-248. 
[113] J. F. Monserrat, R. Fraile, and L. Rubio, "Application of alternating projection method to ensure feasibility of shadowing cross-correlation models," IET Electron. Lett., vol. 43, pp. 724-725, June 2007.

[114] P. J. Rousseeuw and G. Molenberghs, "Transformation of non positive semidefinite correlation matrices," Commun. Stat. A-Theor., vol. 22, pp. 965-984, 1993.

[115] A. J. Viterbi, A. M. Viterbi, and E. Zehavi, "Other-cell interference in cellular power-controlled CDMA," IEEE Trans. Commun., vol. 42, pp. 1501-1504, Feb/Mar/Apr 1994.

[116] A. Viterbi, A. Viterbi, K. Gilhousen, and E. Zehavi, "Soft handoff extends CDMA cell coverage and increases reverse link capacity," IEEE J. Sel. Areas Commun., vol. 12, no. 8, pp. 1281-1288, Oct. 1994.

[117] F. Graziosi, "Analysis of outage duration for mobile radio networks with correlated shadowings," in Proc. IEEE Vehicular Technology Conference, vol. 2, May 1999, pp. 948-952 vol.2.

[118] S. Mukherjee and D. Avidor, "Dynamics of path losses between a mobile terminal and multiple base stations in a cellular environment," IEEE Trans. Veh. Technol., vol. 50, no. 6, pp. 1590-1603, Nov. 2001.

[119] D. Avidor and S. Mukherjee, "Downlink dimensioning for the HSDPA standard," Wireless Pers. Commun., vol. 32, no. 2, pp. 139-152, Jan. 2005.

[120] C. Fischione, F. Graziosi, and F. Santucci, "Approximation for a sum of onoff lognormal processes with wireless applications," IEEE Trans. Commun., vol. 55, no. 10, pp. 1984-1993, Oct. 2007. 
[121] C. Oestges and D. Vanhoenacker-Janvier, "A physical-statistical shadowing correlation model and its application to low-Earth-orbit systems," IEEE Trans. Veh. Technol., vol. 50, no. 2, pp. 416-421, Mar. 2001.

[122] O. Ekici and A. Yongacoglu, "The effect of correlated shadowing on power control error in satellite CDMA systems," in Proc. IEEE Vehicular Technology Conference, vol. 4, May-June 2005, pp. 2643-2647.

[123] C. Oestges and D. Vanhoenacker-Janvier, "Time series-based prediction of fade dynamics and shadowing correlation statistics for navigation systems," in Proc. European Conference on Antennas and Propagation, Nov. 2007, pp. 1-6.

[124] S. Saunders and A. Aragón-Zavala, Antennas and Propagation for Wireless Communication Systems, 2nd ed. John Wiley \& Sons, 2007.

[125] K. Yamamoto, A. Kusuda, and S. Yoshida, "Impact of shadowing correlation on coverage of multihop cellular systems," in Proc. IEEE International Conference on Communications, vol. 10, June 2006, pp. 4538-4542.

[126] H. Kim and Y. Han, "Enhanced correlated shadowing generation in channel simulation," IEEE Commun. Lett., vol. 6, no. 7, pp. 279-281, July 2002.

[127] Z. Lin, X. Peng, K. B. Png, and F. Chin, "Kronecker modelling for correlated shadowing in UWB MIMO channels," in Proc. IEEE Wireless Communications and Networking Conference, Mar. 2007, pp. 1583-1587.

[128] A. Goldsmith, L. Greenstein, and G. Foschini, "Error statistics of real-time power measurements in cellular channels with multipath and shadowing," IEEE Trans. Veh. Technol., vol. 43, no. 3, pp. 439-446, Aug. 1994. 
[129] N. B. Mandayam, P.-C. Chen, and J. M. Holtzman, "Minimum duration outage for cellular systems: A level crossing analysis," in Proc. IEEE Vehicular Technology Conference, vol. 2, Apr. 1996, pp. 879-883.

[130] R. Vijayan and J. Holtzman, "A model for analyzing handoff algorithms," IEEE Trans. Veh. Technol., vol. 42, no. 3, pp. 351-356, Aug. 1993.

[131] D. Giancristofaro, "Correlation model for shadow fading in mobile radio channels," Electron. Lett., vol. 32, no. 11, pp. 958-959, May 1996.

[132] A. A. Abu-Dayya and N. C. Beaulieu, "Outage probabilities in the presence of correlated lognormal interferers," IEEE Trans. Veh. Technol., vol. 43, pp. 164-173, Feb. 1994.

[133] M. Pratesi, M. Ruggieri, F. Graziosi, and F. Santucci, "Performance of signal strength handover algorithms with interference and correlated shadowings," in Proc. IEEE Vehicular Technology Conference, vol. 2, May 1997, pp. 530-534.

[134] D. Baum, J. Hansen, and J. Salo, "An interim channel model for beyond-3G systems: extending the 3GPP spatial channel model (SCM)," in Proc. IEEE Vehicular Technology Conference, vol. 5, May-June 2005, pp. 3132-3136.

[135] I.-K. Fu, C.-F. Li, T.-C. Song, and W.-H. Sheen, "Correlation models for shadow fading simulation," IEEE C802.16m-07/060, Tech. Rep., Mar. 2007.

[136] W. Ni, W. Zou, and H. Wang, "Modeling of spatially cross-correlated shadow fading in distributed radio access networks," in Proc. IEEE International Conference on Communications, May 2008, pp. 4472-4476.

[137] N. B. Mehta, A. F. Molisch, J. Wu, and J. Zhang, "Approximating the sum of correlated lognormal or, lognormal-Rice random variables," in Proc. IEEE International Conference on Communications, vol. 4, June 2006, pp. 1605-1610. 
[138] A. Algans, K. Pedersen, and P. Mogensen, "Experimental analysis of the joint statistical properties of azimuth spread, delay spread, and shadow fading," IEEE J. Sel. Areas Commun., vol. 20, no. 3, pp. 523-531, Apr. 2002.

[139] M. Haleem, D. Avidor, and R. Valenzuela, "Fixed wireless access system with autonomous resource assignment," in Proc. IEEE Internatıonal Symposium on Personal, Indoor and Mobıle Radıo Communıcatıons, vol. 3, Sept. 1998, pp. $1438-1442$.

[140] K. Sayrafian-Pour and D. Kaspar, "A novel model-based indoor positioning using signal strength," in Proc. IEEE Internatıonal Symposium on Personal, Indoor and Mobıle Radıo Communıcatıons, Sept. 2007, pp. 1-5.

[141] D. Kaltakis, "Fundamental limits of GCMAC with fading," Ph.D. dissertation, University of Surrey, Aug. 2009.

[142] X. Yang and R. Tafazolli, "A method of generating cross-correlated shadowing for dynamic system-level simulators," in Proc. IEEE Internatıonal Symposium on Personal, Indoor and Mobıle Radıo Communıcatıons, vol. 1, Sept. 2003, pp. $638-642$.

[143] S. Saunders and B. Evans, "The spatial correlation of shadow fading in macrocellular mobile radio systems," in IEE Colloquzum on Propagation Aspects of Future Mobıle Systems, Oct. 1996, pp. 2/1-2/6.

[144] B. Hochwald, T. Marzetta, and V. Tarokh, "Multiple-antenna channel hardening and its implications for rate feedback and scheduling," IEEE Trans. Inf. Theory, vol. 50, pp. 1893-1909, Sept. 2004. 
[145] I. Kostic, "Analytical approach to performance analysis for channel subject to shadowing and fading," IEE P-Commun., vol. 152, no. 6, pp. 821-827, Dec. 2005.

[146] A. Abdi and M. Kaveh, "On the utility of gamma PDF in modeling shadow fading (slow fading)," in Proc. IEEE Vehıcular Technology Conference, vol. 3, July 1999, pp. 2308-2312.

[147] P. Shankar, "Macrodiversity and microdiversity in correlated shadowed fading channels," IEEE Trans. Veh. Technol., vol. 58, no. 2, pp. 727-732, Feb. 2009.

[148] I. S. Gradshteyn and I. M. Ryzhik, Tables of Integrals, Series, and Products, 7th ed. Academic Press, 2007.

[149] M. C. Cario and B. L. Nelson, "Modeling and generating random vectors with arbitrary marginal distributions and correlation matrix," Dept. Industrial Engineering and Management Sciences, Northwestern University, IL, USA, Tech. Rep., Apr. 1997.

[150] S. Srinivasa, "Modeling interference in uniformly random wireless networks: Theory and applications," Master's thesis, University of Notre Dame, Dec. 2007.

[151] U. Schilcher, M. Gyarmati, C. Bettstetter, Y. W. Chung, and Y. H. Kim, "Measuring inhomogeneity in spatial distributions," in Proc. IEEE Vehıcular Technology Conference, May 2008, pp. 2690-2694.

[152] A. Behnad, H. Purmehdi, and F. Lahouti, "Probability of outage in a clustered Poisson field of interfering nodes," in Proc. IEEE Internatıonal Conference on Telecommunıcatıons, Apr. 2010, pp. 784-789. 
[153] T. K. Sarkar, Z. Ji, K. Kim, A. Medour, and M. Salazar-Palma, "A survey of various propagation models for mobile communication," IEEE Antennas Propagat. Mag., pp. 51-82, June 2003.

[154] P. Harley, "Short distance attenuation measurements at $900 \mathrm{MHz}$ and $1.8 \mathrm{GHz}$ using low antenna heights for microcells," IEEE J. Sel. Areas Commun., vol. 7, pp. 5-11, Jan. 1989.

[155] A. Safak and R. Prasad, "Effects of correlated shadowing signals on channel reuse in mobile radio systems," IEEE Trans. Veh. Technol., vol. 40, no. 4, pp. 708-713, Nov. 1991.

[156] P. Stavroulakis, Interference analysis and reduction for wireless systems. Artech House, 2003.

[157] N. Beaulieu and Q. Xie, "An optimal lognormal approximation to lognormal sum distributions," IEEE Trans. Veh. Technol., vol. 53, pp. 479 489, Mar. 2004.

[158] M. Chiani, A. Conti, and O. Andrisano, "Outage evaluation for slow frequencyhopping mobile radio systems," IEEE Trans. Commun., vol. 47, pp. 1865-1874, Dec. 1999.

[159] S. S. Szyszkowicz, H. Yanikomeroglu, E. Fituri, and S. Periyalwar, "Analytical modeling of interference in cellular fixed relay networks," in Proc. IEEE Canadıan Conference on Electrical and Computer Engıneering, May 2006, pp. 1562-1565.

[160] N. J. Higham, "Analysis of the Cholesky decomposition of a semi-definite matrix," in Proc. Relable Numerical Computatıon. University Press, 1990, pp. $161-185$. 
[161] B. Alkire, "Cholesky factorization of augmented positive definite matrices," Electrical Engineering Department, UCLA, Tech. Rep., Dec. 2002.

[162] J. Geist, "Computer generation of correlated Gaussian random variables," Proc. IEEE, vol. 67, no. 5, pp. 862-863, May 1979.

[163] A. Lukin, "Tips \& tricks: Fast image filtering algorithms," in Proc. Internatıonal Conference on Computer Graphıcs 63 Visıon, June 2007, pp. 186-189.

[164] J.-J. Wu, "Simulation of rough surfaces with FFT," Trrbol. Int., vol. 33, pp. 47-58, Feb. 2000.

[165] B. Kozintsev, "Computations with Gaussian random fields," Ph.D. dissertation, University of Maryland at College Park, 1999.

[166] T. Preis, P. Virnau, P. Wolfgang, and J. J. Schneider, "GPU accelerated Monte Carlo simulation of the 2D and 3D Ising model," J. Comput. Phys., vol. 228, pp. 4468-4477, Mar. 2009.

[167] R. Bhagavatula, C. Oestges, and R. Heath, "A new double-directional channel model including antenna patterns, array orientation, and depolarization," IEEE Trans. Veh. Technol., vol. 59, no. 5, pp. 2219-2231, June 2010.

[168] J. Salo, "A sum-product model as a physical basis for shadow fading," CoRR, vol. abs/cs/0702098, 2007.

[169] K. Rizk, A. Mawira, J.-F. Wagen, and F. Gardiol, "Propagation in urban microcells with high rise buildings," in Proc. IEEE Vehıcular Technology Conference, Apr. 1996, pp. 859-863.

[170] H. R. Anderson, "Building corner diffraction measurements and predictions using UTD," IEEE Trans. Antennas Propag., vol. 46, no. 2, pp. 292-293, Feb. 1998. 
[171] V. Degli-Esposti, G. Lombardi, C. Passerini, and G. Riva, "Wide-band measurement and ray-tracing simulation of the $1900-\mathrm{MHz}$ indoor propagation channel: Comparison criteria and results," IEEE Trans. Antennas Propag., vol. 49, no. 7 , pp. 1101-1110, July 2001.

[172] B. Neekzad, K. Sayrafian-Pour, J. Perez, and J. Baras, "Comparison of ray tracing simulations and millimeter wave channel sounding measurements," in Proc. IEEE Internatıonal Symposıum on Personal, Indoor and Mobıle Radıo Communıcatıons, Sept. 2007, pp. 1-5. 